SANDIA REPORT

SAND94-2589 • UC-406 706

Unlimited Release

Printed January 1997

RECEIVED

MAR O 51997

OSTI

\title{
Optical Diagnostics for Turbulent and Multiphase Flows: Particle Image Velocimetry and Photorefractive Optics
}

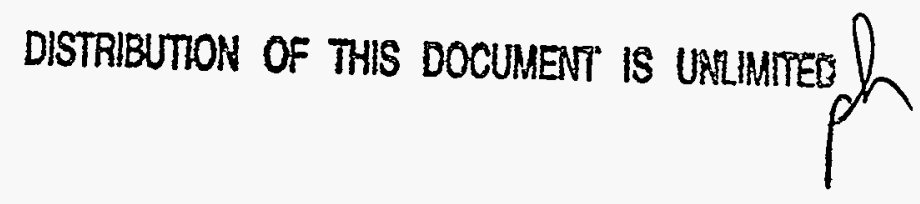

T.J. O'Hern, J.R. Torczynski, R.N. Shagam, T.K. Blanchat, T.Y. Chu

A.L. Tassin-Leger, J.A. Henderson

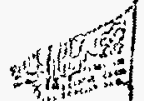

Sandia National Laboratories

Albuquerque, New Mexico 87185 and Livermore, California 94550

for the United States Depaintment of Energy

under Contract DE-AC04-94AL85000,

III!I!

?

min

Approved for públic rélease; distribution is unlimited.
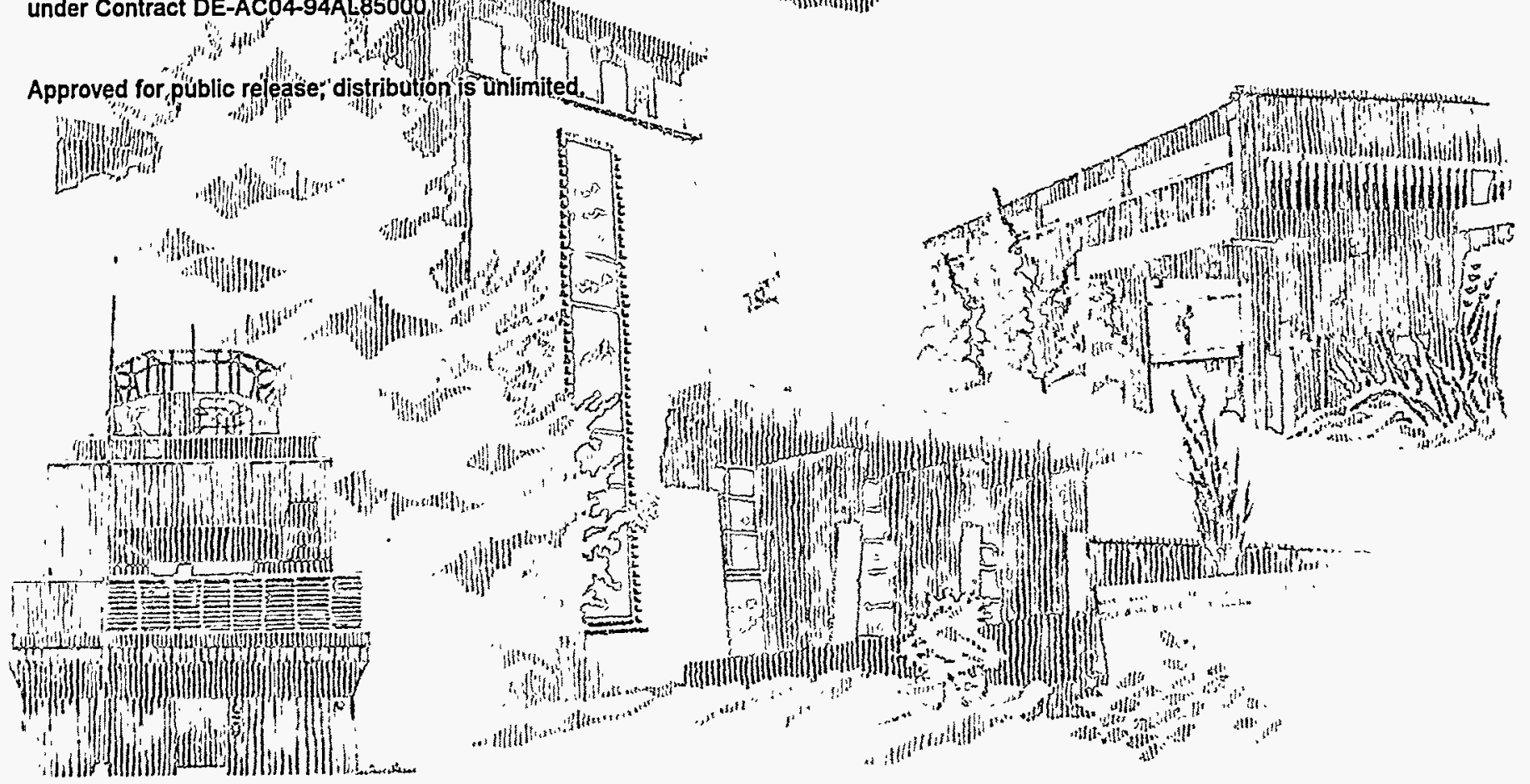

SF2900Q(8-81) 
Issued by Sandia National Laboratories, operated for the United States Department of Energy by Sandia Corporation.

NOTICE: This report was prepared as an account of work sponsored by an agency of the United States Government. Neither the United States Government nor any agency thereof, nor any of their employees, nor any of their contractors, subcontractors, or their employees, makes any warranty, express or implied, or assumes any legal liability or responsibility for the accuracy, completeness, or usefulness of any information, apparatus, product, or process disclosed, or represents that its use would not infringe privately owned rights. Reference herein to any specific commercial product, process, or service by trade name, trademark, manufacturer, or otherwise, does not necessarily constitute or imply its endorsement, recommendation, or favoring by the United States Government, any agency thereof, or any of their contractors or subcontractors. The views and opinions expressed herein do not necessarily state or reflect those of the United States Government, any agency thereof, or any of their contractors.

Printed in the United States of America. This report has been reproduced directly from the best available copy.

Available to DOE and DOE contractors from

Office of Scientific and Technical Information

P.O. Box 62

Oak Ridge, TN 37831

Prices available from (615) 576-8401, FTS 626-8401

Available to the public from

National Technical Information Service

U.S. Department of Commerce

5285 Port Royal Rd

Springfield, VA 22161

NTIS price codes

Printed copy: A05

Microfiche copy: A01 


\section{DISCLAIMER}

Portions of this document may be illegible in electronic image products. Images are produced from the best available original document. 
SAND 94-2589

Distribution

Unlimited Release

Category UC-406 706

Printed January 1997

\title{
Optical Diagnostics for Turbulent and Multiphase Flows: Particle Image Velocimetry and Photorefractive Optics
}

\author{
T. J. O'Hern, J. R. Torczynski, R. N. Shagam*, T. K. Blanchat**, T. Y. Chu**, \\ A. L. Tassin-Leger, and J. A. Henderson \\ Engineering Sciences Center \\ *Surety Components and Instrumentation Center. \\ **Nuclear Energy Technology Center
}

Sandia National Laboratories

P. O. Box 5800

Albuquerque, New Mexico 87185-0826

\begin{abstract}
This report summarizes the work performed under the Sandia Laboratory Directed Research and Development (LDRD) project "Optical Diagnostics for Turbulent and Multiphase Flows."Advanced optical diagnostics have been investigated and developed for flow field measurements, including capabilities for measurement in turbulent, multiphase, and heated flows. Particle Image Velocimetry (PIV) includes several techniques for measurement of instantaneous flow field velocities and associated turbulence quantities. Nonlinear photorefractive optical materials have been investigated for the possibility of measuring turbulence quantities (turbulent spectrum) more directly.

The two-dimensional PIV techniques developed under this LDRD were shown to work well, and were compared with more traditional laser Doppler velocimetry (LDV). Three-dimensional PIV techniques were developed and tested, but due to several experimental difficulties were not as successful. The photorefractive techniques were tested, and both potential capabilities and possible problem areas were elucidated.
\end{abstract}




\section{Acknowledgment}

This work was supported by the United States Department of Energy under Contract DE-AC0494AL85000. Sandia is a multiprogram laboratory operated by Sandia Corporation, a Lockheed Martin Company, for the United States Department of Energy. The support of DOE/Sandia Laboratory-Directed Research and Development (LDRD) funds is gratefully acknowledged. The technical assistance of T. W. Grasser in optical configuration, flow facility setup and maintenance, and data acquisition, is greatly appreciated. V. A. Amatucci assisted on the nonlinear optics evaluation. 


\section{Table of Contents}

Acknowledgment

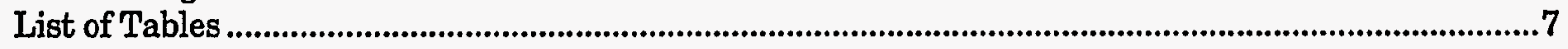

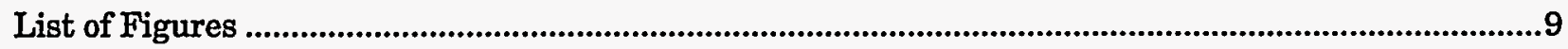

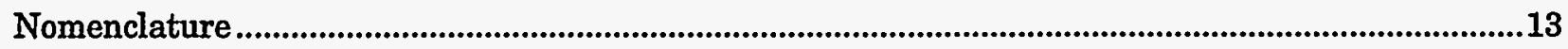

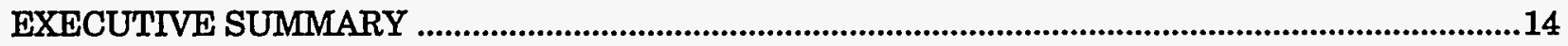

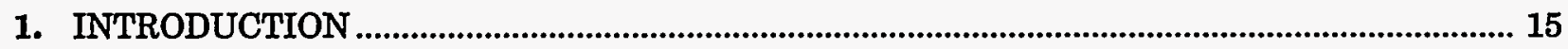

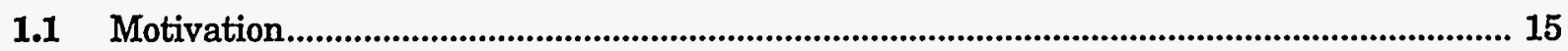

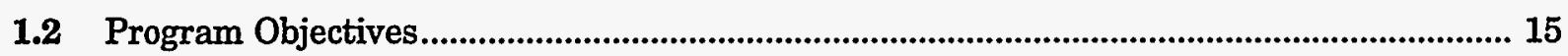

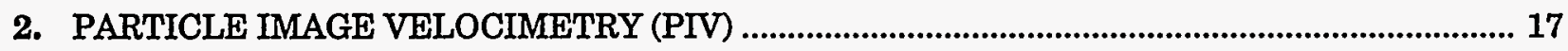

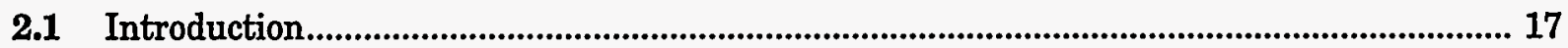

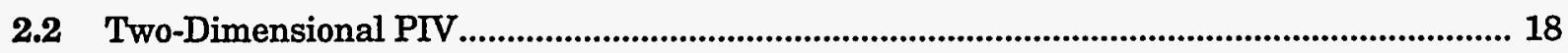

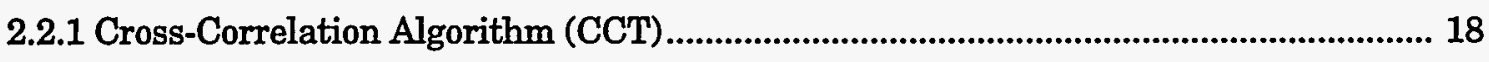

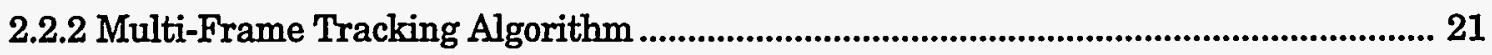

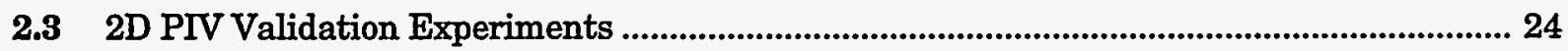

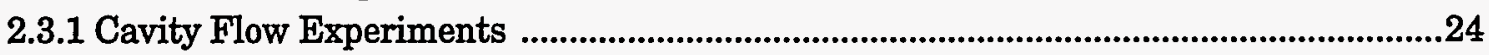

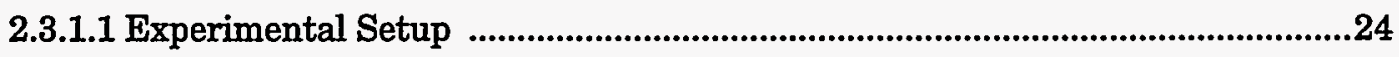

2.3.1.2 Experimental Techniques ......................................................................................26

2.3.1.2.1 Laser Doppler Velocimetry (LDV) ......................................................26

2.3.1.2.2 Particle Image Velocimetry (PIV)' ....................................................27

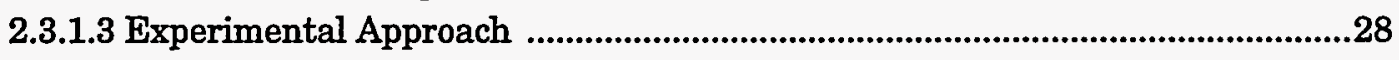

2.3.1.4 Computational Approach …...............................................................................29

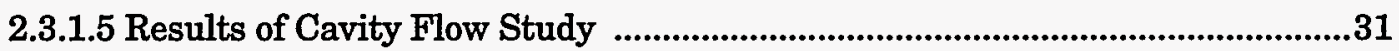

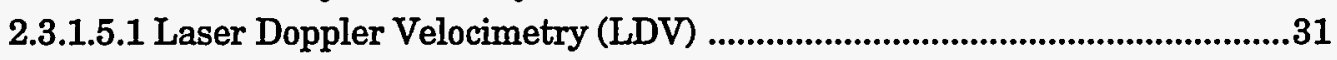

2.3.1.5.2 Particle Image Velocimetry (PIV) ......................................................31

2.3.1.5.3 Computational Simulations (FIDAP) ........................................................34

2.3.1.5.4 Comparison of Results ................................................................................38

2.3.1.6 Summary and Conclusions .................................................................39

2.3.2 2D Thermal Convection Experiments .......................................................................40

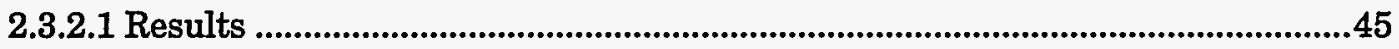

3. THREE-DIMENSIONAL PARTICLE IMAGE VELOCIMETRY …........................................49

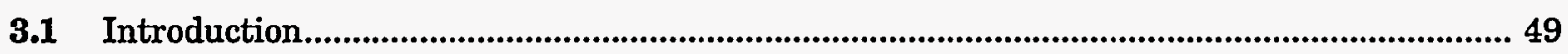

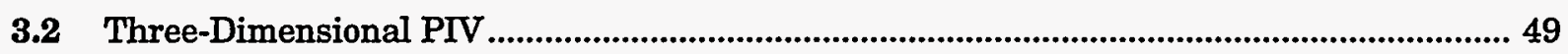

3.2.1 Camera Calibration and Particle Combination Theory ..................................................49

3.2.2 Theory for Tracking Programs ............................................................................55

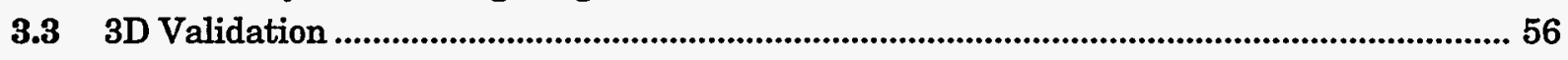

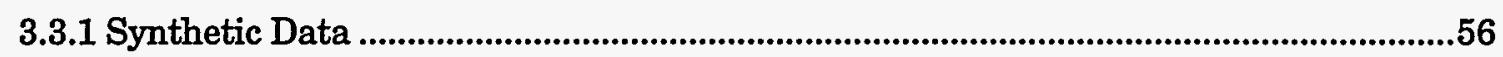

3.3.2 Three-Dimensional Convection Experiment Set-Up ......................................................57

3.3.3 Results of Three-Dimensional Experiments ...............................................................57

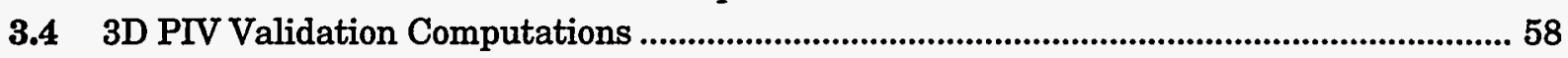

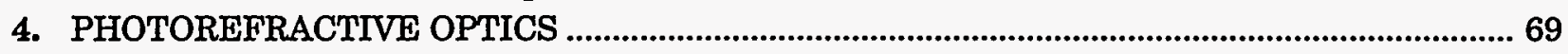

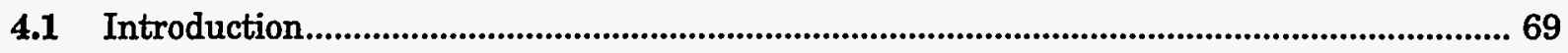

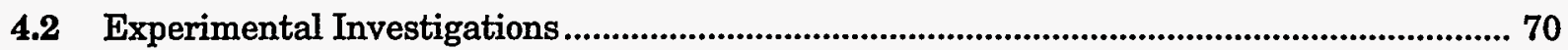




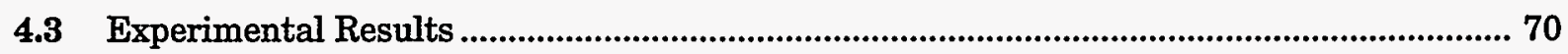

4.3.1 Turbulent Power Spectral Density Measurements .................................................70

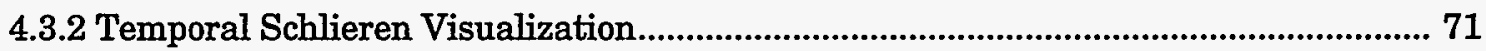

4.3.3 Orientation Effects on Flow Visualization ...................................................................72

4.3.4 Sensitivity to Wavefront vs. Slope Disturbances ........................................................ 73

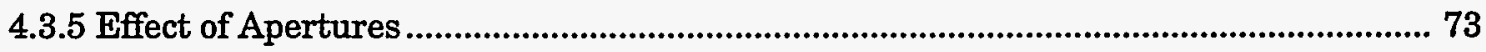

4.3.6 Temporal Transmission Measurements ...................................................................... 73

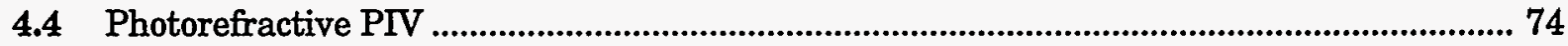

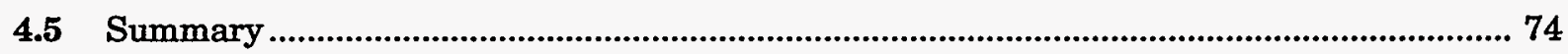

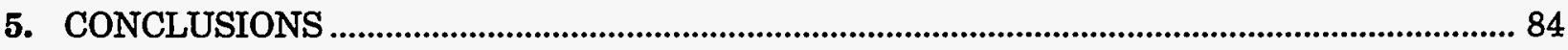

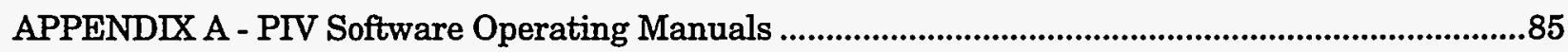

A. 1. Instructions for Using PIV Software for 2D Analysis.......................................................... 85

A. 2. Instructions for Using PIV Software for 3D Analysis.................................................... 86

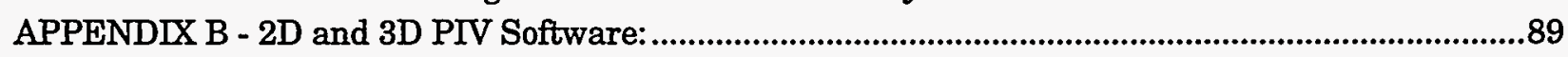

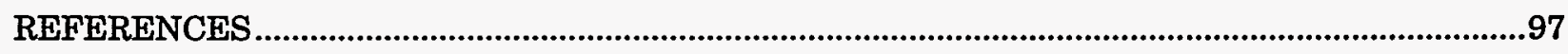

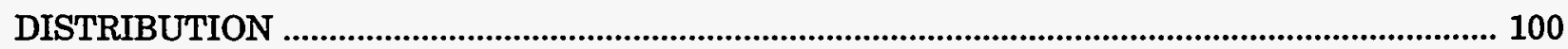




\section{List of Tables}

Table 2.2.1. Nondimensionalization of properties......................................................................... 59

Table 2.2.2. Nondimensionalization of variables .............................................................................59

Table 2.2.3. Dimensionless parameters …..........................................................................................59 
This page left intentionally blank 


\section{List of Figures}

Figure 2.1 Calculating $C_{\mathrm{ij}}$ value for Cross Correlation Tracking (CCT) technique..................... 19

Figure 2.2 Intersection of particle images $i$ and $j$ between frames 1 and 2 . ....................................

Figure 2.3 Determination of possible track for Multiframe Tracking (MFT) technique. .............22

Figure 2.4 Schematic diagram of water channel/cavity flow. ....................................................25

Figure 2.5 Schematic layout of cavity flow system.D $=3.68 \mathrm{~cm}, \mathrm{~L}=3.70 \mathrm{~cm}, \mathrm{~h}=1.56 \mathrm{~cm}, \mathrm{w}=$

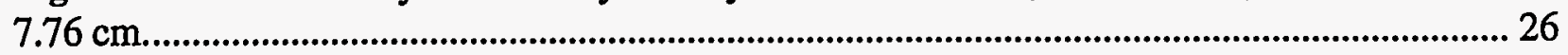

Figure 2.6 Schematic diagram of computational domain. Channel flow is from lower right to upper left. Symmetry plane (dashed) is exposed to view. .................................................................. 29

Figure 2.7 Computational meshes (27-node brick elements): top, coarse; bottom, refined. ........30

Figure 2.8 LDV results for cavity flow. Time-averaged centerplane velocity fields. ....................32

Figure 2.9 PIV results for cavity flow. Instantaneous centerplane velocity fields. ........................33

Figure 2.10 CFD simulations of velocity vectors in the cavity flow at $\operatorname{Re}=600$. Flow above the cavity is from left to right, with a peak velocity of about $2.8 \mathrm{~cm} / \mathrm{s}$.

Figure 2.11 Dimensionless Re-dependence of position $\left(X_{C}, Y_{C}\right)$ of center of main eddy in the symmetry plane $\mathrm{z}=0$.

Figure 2.12 Streamlines: above, $\operatorname{Re}=3$ (no isolated region); below, $\operatorname{Re}=30$ (isolated region). 36

Figure 2.13 Topologies: left, low Re; right, high $R e$. Transition occurs at $\operatorname{Re}=27$.

Figure 2.14 Comparison of LDV and PIV velocity profiles through the center of primary recirculation region in cavity, for $\mathrm{Re}=100$ and $\mathrm{Re}=600$.

Figure 2.15 Conceptual representation of a single well of a magma-energy extraction system in operation (cf. Chu et al., 1990).

Figure 2.16 Experimental setup for thermal convection experiments. Dimensions are inches....42

Figure 2.17 Schematic diagram of cooling cylinder inserted into convection tank. . .43

Figure 2.18 2-D PIV setup for thermal convection experiments.

Figure 2.19 Laser light sheet flow-visualization photographs: (a) slice through cylinder along heater; (b) slice through cylinder across heater.

Figure 2.20 PIV velocity vectors at similar conditions. Left, slice through cylinder along heater strip (plane $x=0$ ); right, slice through cylinder across heater plane (plane $y=0$ ). The flow pattern agrees with the flow visualization. Flow is downward beneath cylinder.. . .46

Figure 2.21 Finite element CFD simulation of selected streamlines in the symmetry planes......47

Figure 2.22 Streamlines calculated from PIV velocity fields given in Figure 2.20. Qualitative agreement with computational simulation in Figure 2.21 can be seen.

Figure 3.1 Three-Dimensional PIV set-up. . .50 


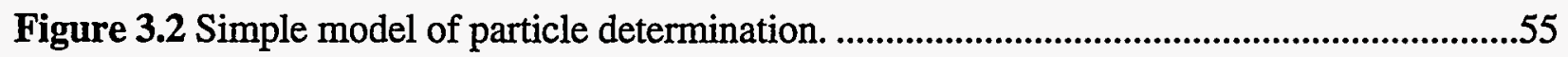

Figure 3.3 Schematic diagrams showing different views of the geometry. ...............................61

Figure 3.4 Left, coarse mesh. Right, refined mesh. ..............................................................62

Figure 3.5 Effect of mesh on $\mathrm{u}_{\mathrm{z}}$ and $\mathrm{T}$ along $\mathrm{x}=\mathrm{y}=0$ (below the cylinder on its axis). ............62

Figure 3.6 Selected streamlines on symmetry planes. .................................................................. 63

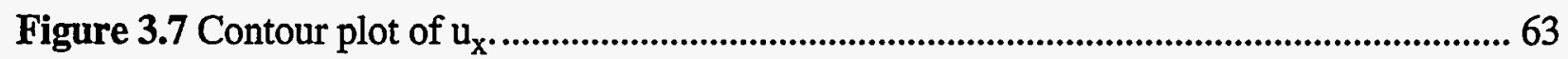

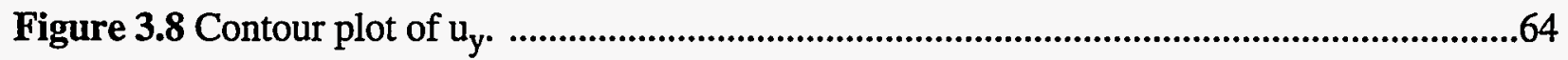

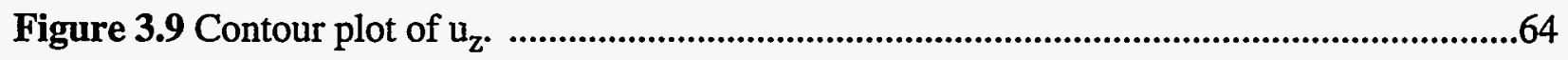

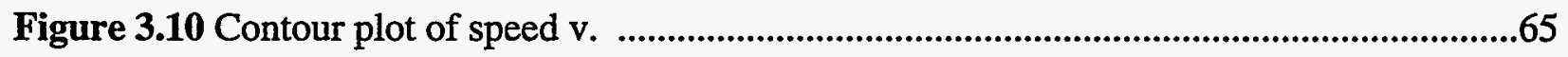

Figure 3.11 Contour plot of pressure p. ................................................................................65

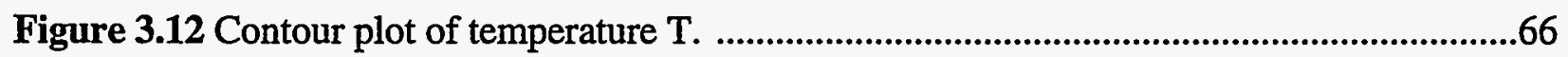

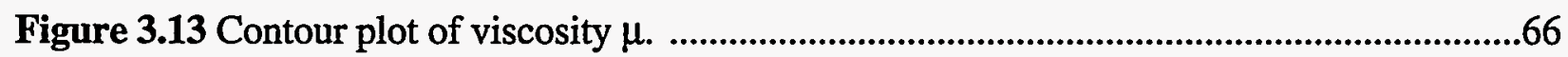

Figure 3.14 Computational results: left, temperature contours; center, velocity vectors; right, selected streamlines on symmetry planes.

Figure 3.15 Laser light sheet flow-visualization photographs: (a) slice through cylinder along heater; (b) slice through cylinder across heater.

Figure 4.1 Schematic layout of optical system for power spectral density (PSD) measurements. Fourier lens $500 \mathrm{~mm}$ focal length, relay lens $125 \mathrm{~mm}$ focal length. Video camera images Fourier plane of relay lens.

Figure 4.2 Turbulent power spectral density (PSD) measurements. (A) Static background image (no flow). (B) Gas jet: low-flow conditions. (C) Gas jet: high-flow conditions.

Figure 4.3 Schematic layout for photorefractive schlieren technique. Video camera images the phase disturbance plane.

Figure 4.4 Photorefractive schlieren flow visualization with $\mathrm{BaTiO}_{3}$. (A) Background field, no flow. (B) $\mathrm{CO}_{2}$ jet flow field. (C) Jet flow field with background subtracted. ..................................76

Figure 4.5 Effect of flow perturbation on $\mathrm{BaTiO}_{3}$. Test object is hot soldering iron. (A) Static laminar flow. (B) Perturbed flow field (air currents). (C) Perturbed flow field with static flow subtracted.

Figure 4.6 Orientation dependence of a turbulent $\mathrm{CO}_{2}$ jet in air.(A)Horizontal flow.(B) Vertical flow. (C) Intensity histograms. ..................................................................................................... 78

Figure 4.7 Dependence of photorefractive effect on gradient orientation. (A, B) Cross flow. (C, D) With flow. Crystal fanning is in vertical direction. .......................................................................79

Figure 4.8 Schematic of phase sensitivity test setup. ...................................................................79

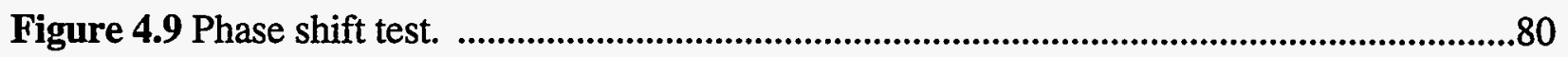


Figure 4.10 Effect of cross-coupling beams within crystal. The arrows point to regions of temporally modulated fringes.

Figure 4.11 Effect of a vertical aperture on wavefront extinction: fanning direction vertical. ...81 Figure 4.12 Effect of a horizontal aperture on wavefront extinction: fanning direction vertical. 81 Figure 4.13 Crystal extinction vs. time for a variety of initial poling (erasure exposure) times. Incident laser power constant. 82

Figure 4.14 Crystal peak transmission vs. poling time. ................................................................83

Figure A.1 Basic outline for PIV software for 2D and 3D analysis ..............................................8 
This page left intentionally blank 


\section{Nomenclature}

$\mathrm{C}_{\mathrm{ij}} \quad$ Correlation coefficient - used in cross-correlation tracking technique

CCT Cross-Correlation Tracking

$\mathrm{D}_{\mathrm{ij}} \quad$ Amount that the diameters of overlapping particles $\mathrm{i}$ and $\mathrm{j}$ - used in cross-correlation tracking technique

MFT Multi-Frame Tracking algorithm

$\mathrm{N}_{\mathrm{ij}} \quad$ Number of particles that overlap between Images $\mathrm{i}$ and $\mathrm{j}$ - used in cross-correlation tracking technique

$\mathrm{R}_{\mathrm{ij}} \quad$ pair reliability index - used in cross-correlation tracking technique

$R_{n} \quad$ Radius of search circle - used in multi-frame tracking technique

$\operatorname{Re} \quad$ Reynolds number for cavity flow $=\overline{\mathrm{u}}(2 \mathrm{~h}) / \mathrm{v}$

D Cavity Depth $=3.68 \mathrm{~cm}$ (Figure 2.5 )

L Cavity Length in Streamwise direction $=3.70 \mathrm{~cm}$ (Figure 2.5)

$\mathrm{h}$ channel depth upstream of cavity $=1.56 \mathrm{~cm}$ (Figure 2.5)

w channel/cavity cross-stream width $=7.76 \mathrm{~cm}$ (Figure 2.5 )

$\mathrm{u}_{\mathrm{x}} \quad$ velocity component in the $\mathrm{x}$ direction

$\mathrm{u}_{\mathrm{y}} \quad$ velocity component in the $\mathrm{y}$ direction

$\mathrm{u}_{\mathrm{z}} \quad$ velocity component in the $\mathrm{z}$ direction

$u_{\mathrm{av}} \quad$ channel mass-flow velocity

$\mathrm{X}_{\mathrm{C}} \quad \mathrm{X}$ center of main eddy in cavity flow (Figure 2.11)

$\mathrm{Y}_{\mathrm{C}} \quad \mathrm{Y}$ center of main eddy in cavity flow (Figure 2.11)

$\sigma_{1} \quad$ Standard deviation from the mean for the lengths of the vectors

$\sigma_{\theta} \quad$ Standard deviation from the mean for the angles in the x-y plane

$\sigma_{\Phi} \quad$ Standard deviation from the mean for the angles from the $\mathrm{z}$ axis

$\sigma \quad$ Total standard deviation

$\mathrm{L}_{\mathrm{x}-\mathrm{y}} \quad$ Length between particles between frames $\mathrm{x}$ and $\mathrm{y}$

$\theta_{1-3} \quad$ Angle in the $x-y$ plane between $\mathrm{I}_{1-2}$ and $\mathrm{l}_{2-3}$

$\theta_{2-4} \quad$ Angle in the $x-y$ plane between $\mathrm{l}_{2-3}$ and $\mathrm{l}_{3-4}$

$\Phi_{1-3} \quad$ Angle from the $\mathrm{z}$ axis between $\mathrm{I}_{1-2}$ and $\mathrm{l}_{2-3}$

$\Phi_{2-4} \quad$ Angle from the $\mathrm{z}$ axis between $\mathrm{I}_{2-3}$ and $\mathrm{l}_{3-4}$

$\overline{\mathrm{L}} \quad$ Average displacement of particles between frames $=\left(\mathrm{L}_{1-2}+\mathrm{L}_{2-3}+\mathrm{L}_{3-4}\right) / 3$

$\bar{\theta} \quad$ Average $\theta$ of the track $=\left(\theta_{1-3}+\theta_{2-4}\right) / 2$

$\bar{\Phi} \quad$ Average $\Phi$ of the track $=\left(\Phi_{1-3}+\Phi_{2-4}\right) / 2$ 


\section{Executive Summary}

This report summarizes the work performed under the Sandia Laboratory Directed Research and Development (LDRD) project “Optical Diagnostics for Turbulent and Multiphase Flows.”

Advanced optical diagnostics have been investigated and developed for flow field measurements, including capabilities for measurement in turbulent, multiphase, and heated flows. Particle Image Velocimetry (PIV) includes several techniques for measurement of instantaneous flow field velocities and associated turbulence quantities. Nonlinear photorefractive optical materials have been investigated for the possibility of measuring turbulence quantities (turbulent spectrum) more directly.

The two-dimensional PIV techniques developed under this LDRD were demonstrated to work well and were compared with more traditional laser Doppler velocimetry (LDV), and with computational simulations. Two-dimensional benchmarking experiments included flow in a driven cavity and natural convection in a simulated magma chamber. Three-dimensional PIV techniques were developed and tested on the thermal convection experiment but were unsuccessful due to several experimental difficulties.

The nonlinear photorefractive crystal $\mathrm{BaTiO}_{3}$ has been demonstrated as a significant visualizer of turbulent vs. steady-state flow. However, we have identified two potentially significant limitations in that it (1) appears to visualize gradients differently, depending upon the relative orientation of the gradient to the crystal fanning orientation, and (2) visualizes refractive gradients, not absolute phase change, as previously reported in the literature. The desired quantification of photorefractive measurements proved to be very difficult for these reasons. 


\section{INTRODUCTION}

\subsection{Motivation}

Application of optical diagnostics to the field of fluid mechanics has proven to be invaluable in advancing the state of the art. Optical diagnostics are ideally suited to examination of many fluid flows, offering the advantages of noninvasive probing of flow fields, obtaining full-field in additional to point measurements, utilizing computational-optics techniques like optical Fourier transforms, and applying the wealth of readily available, well-developed image-processing techniques. From the early days of flow visualization in transparent fluids, optical diagnostics have become increasingly quantitative and now include such widely accepted techniques as laser Doppler velocimetry (LDV) and holographic interferometry. However, there are still needs for advanced diagnostics, especially for measurements in multiphase and turbulent flows. These are very important flow categories, as they encompass most environmental and industrial flows. In addition, multiphase and turbulent flows are difficult to simulate computationally, so high-quality measurements are in great need, both for fundamental understanding of the flow physics and for developing and validating advanced computational techniques. The noninvasive nature of optical techniques is particularly important in turbulent and multiphase flows, where probes may interact with the turbulence to change the flow properties or may interact differently with different components of a multiphase flow.

\subsection{Program Objectives}

The goal of this work was to develop optical diagnostic techniques to provide Sandia with advanced flow-measurement capabilities. The first technique developed and evaluated is Particle Image Velocimetry (PIV), in which a flow seeded with small particles is photographed repeatedly at short time intervals. Image-processing techniques are applied to find particle velocities from particle positional changes in successive frames, which permits determination of the instantaneous flow field and associated turbulence quantities. Standard PIV uses a laser light sheet to illuminate a plane of the flow for 2D measurements, while volume illumination and multiple cameras are used for 3D PIV. The second technique developed and evaluated uses nonlinear photorefractive optical crystals to measure turbulence quantities more directly. A laser beam passed through the flow acquires a small perturbation signal related to the turbulence, which is superimposed on a large steady informationless signal, rendering interpretation difficult. The laser beam is then passed through one or more crystals to remove most of the steady portion, thereby greatly improving the signal-to-noise ratio. Since turbulence models are at best of questionable validity and often difficult to extend to new flow situations, this capability will enable us to assess and potentially improve the accuracy of numerical turbulence models by directly measuring turbulence quantities to compare with model predictions. 
The flows of interest for application of these new diagnostics are typically turbulent, multiphase, and/or nonuniformly heated, making traditional point measurements difficult and unreliable. These new diagnostics provide unique capabilities to make such measurements in a noninvasive fashion. 


\section{PARTICLE IMAGE VELOCIMETRY (PIV)}

\subsection{Introduction}

Particle Image Velocimetry (PIV) is used to measure two-dimensional velocity fields by analyzing the motion of flow-tracing seed particles, recorded in photographic or video images (e.g., Adrian, 1991). This technique promises to become one of the most useful in experimental fluid mechanics. In a PIV experiment, a flow seeded with small particles is photographed repeatedly at short time intervals, typically using a laser light sheet to illuminate particles only in the plane of interest. Image-processing techniques are applied to find particle velocities from particle positional changes in successive frames, which permits determination of the instantaneous flow field and associated turbulence quantities. This near-instantaneous, full-field velocity mapping complements such point velocity measurement techniques as laser Doppler velocimetry and hot-wire anemometry. Where these techniques are capable of providing detailed velocity data at a point in the flow, they can only be used to map out the velocity field in steady-state flows. However, full-field velocity data are acquired very quickly using the PIV technique, allowing velocity field mapping in unsteady flows. Quantities such as vorticity and strain rates in fluids are very difficult to determine using point measurement techniques since they involve derivatives of the velocity field, which require measurements of very fine spatial resolution. PIV measurements allow determination of such quantities since they are of sufficiently fine spatial resolution.

PIV is often referred to as a "quantitative flow visualization" technique since the data record is typically a high-quality photograph or video of the flow. The human eye is very good at tracking particles between frames of a multiframe sequence, or even performing the autocorrelation to determine particle motion in multiple-exposure images. Velocity quantification in PIV is done by automating this process, using a computer to measure the displacement of individual particles in the flow field, over a known time period, using one of several different algorithms (see, e.g., Lourenco et al. 1989; Grant 1994). Two different PIV algorithms will be presented in this chapter, one based on frame-to-frame cross-correlation, the other based on frame-to-frame particle tracking.

Among its other advantages, PIV is especially useful in multiphase flows, at least for those cases where the separate phases can be tracked individually. For example, in a gas-liquid two-phase flow, small solid flow-tracing seed particles could be used to track the liquid velocity, while bubbles could be directly tracked to give gas velocity (Hassan and Blanchat, 1991a). In turbulent flows, the fast data acquisition of PIV holds promise for measuring rapid fluctuations throughout a flow field. However, turbulence statistics must be built up by working with a large number of frames.

At the time that this LDRD work was undertaken, there were no commercial sources for PIV systems. At present, there are at least three companies offering PIV hardware, software, and/or full systems. They include TSI Inc. (St. Paul, MN), Dantec Inc. (Mahwah, NJ), and Oxford Lasers (Acton, MA). 


\subsection{Two-Dimensional PIV}

One of the first decisions to be made when setting up a PIV system is the data acquisition method, usually either a film camera or an electronic (usually CCD) camera. The primary advantage of a film camera system is better resolution. However, there is a trade-off between resolution and acquisition speed. High-speed motion picture cameras typically operate with smaller film sizes (e.g., $16 \mathrm{~mm}$ format), so resolution is lowered. For higher-resolution film cameras, the film advance times limit the data acquisition speed for sequential images. For this reason, multiple exposures on a single film frame are often used. An autocorrelation is then used to determine particle motion, but there is a directional ambiguity unless special image-shifting techniques are applied. The use of film also involves the time-consuming step of darkroom processing before image quality can be determined. PIV analysis of multiple-exposure film images is sometimes done using the Young's fringe analysis, where a probe laser beam traverses the image (usually the photographic negative), and the spacing and orientation of the diffraction fringe pattern is measured to determine local velocity (magnitude and direction).

Electronic image capture is usually limited to 30 frames per second for a conventional RS-170 video signal, at lower resolution than available with film. The main advantage of an electronic camera is immediate feedback, very useful for determining illumination levels, camera settings, seed density, etc. Other advantages are that the picture can be directly stored as a binary computer file available for image processing and measurement, and that there is no directional ambiguity when a sequence of frames is used. Both film and CCD cameras have been used in this work, although primarily the latter.

Light sheets for planar illumination can be formed either by expanding a laser beam with cylindrical optics, or by rapidly sweeping a focused laser beam through the flow field during exposure, forming an effective light sheet. Both planar illumination techniques have been applied for this work.

Two methods have been developed to obtain the displacement of multiple particles between flow images. The first is the cross-correlation tracking (CCT) algorithm, and the second is the multiframe particle tracking (MFT) algorithm. These two methods are used both for 2D PIV as discussed herein and for the 3D PIV discussed in Chapter 3 (programs PST3D and TP3D do crosscorrelation and particle tracking, respectively, for both 2D and 3D PIV). The programs are written in Fortran, and are based on the graduate work of two of the authors (TKB and JAH) at Texas A\&M University (cf. Blanchat, 1991; Hassan and Blanchat, 1991ab)

\subsubsection{Cross-Correlation Algorithm (CCT)}

Cross-correlation tracking (CCT) is a dynamic particle tracking method that can be performed between two sequential digital images. The particle velocity is found by determining the correspondence between particles in the comparison areas of the two images. Every particle belongs to a characteristic group that has a specific local 2D distribution pattern in the first image and multiple 
Frame 1 Search Region
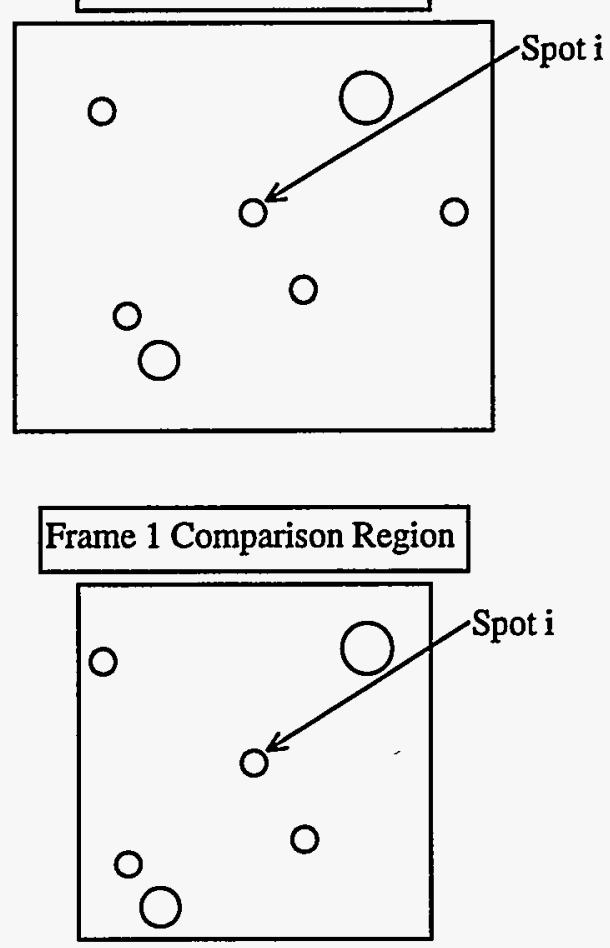

Frame 2 Search Region
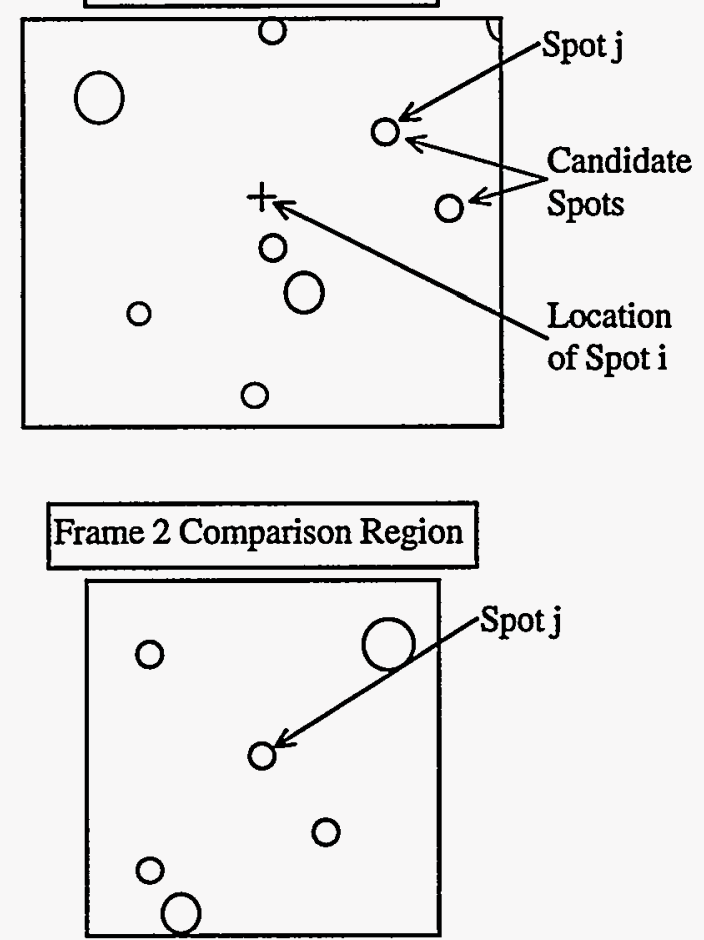

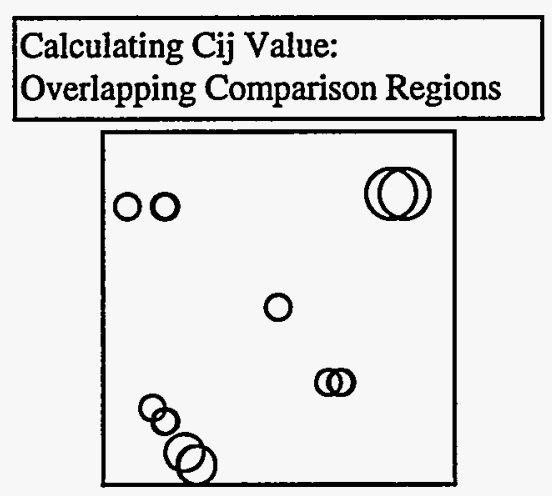

Figure 2.1. Calculating $C_{\mathrm{ij}}$ value for Cross Correlation Tracking (CCT) technique.

candidate patterns in the second image. Each second image candidate's comparison area is shifted over the first image comparison area such that the original particle's center is positioned over the candidate particle's center. The particle in the first image corresponds to the particle in the second image that maintains the most similar pattern within the comparison area, providing that the local pattern of the distributed particles changes little within the time that the original images were taken. This method is particularly useful when only two sequential images are available, and the multiframe tracking method (see Section 2.2.2) cannot be performed. 
The CCT algorithm correlates between two frames of data. A search area is formed in the second frame, centered on the location of the original particle in the first frame. The size of this area is determined by estimating the maximum possible movement for particles between frames. Each Frame 2 particle in this area is a candidate for being the original particle as moved by the flow. A comparison region is then created around each candidate, encompassing at least ten other particles in Frame 2. A comparison area of the same size is created in Frame 1, centered on the original Frame 1 particle. The two areas are then compared by shifting the center of the candidate particle over the center of the original particle. Figure 2.1 demonstrates this procedure. This comparison is characterized by the correlation coefficient, $\mathrm{C}_{\mathrm{ij}}$, which measures the overlap of the particles in the two areas. Figure 2.2 shows how the overlap of the diameters of a Frame 1 and Frame 2 spot is measured. $\mathrm{A} \mathrm{C}_{\mathrm{ij}}$ value of 1 indicates a perfect correspondence between two areas; the closer the value is to 1 , the more likely that it is the correct particle match. $\mathrm{A}_{\mathrm{ij}}$ value of 1 is possible only if each spot is the same size in both comparison areas, and each spot is centered on the same point when shifted to overlap the two areas. Equation 1 is used to calculate the correlation coefficient between the comparison areas for frames 1 and 2:

$$
\mathrm{C}_{\mathrm{ij}}=\frac{\sum_{i=1}^{P_{1}} \sum_{j=1}^{P_{2}} \mathrm{D}_{i, 1} \cap \mathrm{D}_{j, 2}}{\sqrt{\left(\sum_{i=1}^{P_{1}} \mathrm{D}_{i, 1}\right)\left(\sum_{j=1}^{P_{2}} \mathrm{D}_{j, 2}\right)}}
$$

where the numerator is the number of overlapping pixels between the two frames, and the denominator is the geometric mean number of total particle pixels in the two frames. The maximum value of $\mathrm{C}_{\mathrm{ij}}$ is therefore equal to one. In other words, $\mathrm{C}_{\mathrm{ij}}$ is simply the ratio of the overlapping "on" pixels (i.e., particle present) in the two frames to the total possible number (Blanchat, 1991). The particle pair with the highest $C_{i j}$ value is then identified as the best particle pair match. Given a purely translational flow and a sufficiently small comparison region, $\mathrm{C}_{\mathrm{ij}}$ values obtained for the correct particle match are much greater than those of incorrect matches. In areas of high vorticity, or if the comparison region is too large, the difference in $C_{\mathrm{ij}}$ values can be much smaller, and the identified matches are not always correct. Another means of checking is required to remove erroneous particle pairs. Therefore, two more checks are performed. The first check calculates the sum of the distances between all corresponding centroid pairs. For perfect overlap of all particles in the Frame 1 and Frame 2 search areas, this value will be zero.

The second check determines a reliability index for a possible pair based on the number of particles that overlap, $\mathrm{N}_{\mathrm{ij}}$, and the amount the diameters of those particles overlap, $\mathrm{D}_{\mathrm{ij}}$, which occur when checking the correspondence between spot $i$ and $j$. This overlap was also used in calculating the $C_{i j}$ value and is displayed in Figure 2.2. When all correspondences are completed, the pair reliability index $R_{i j}$ is calculated as follows:

$$
R_{i j}=N_{i j} \times D_{i j}
$$




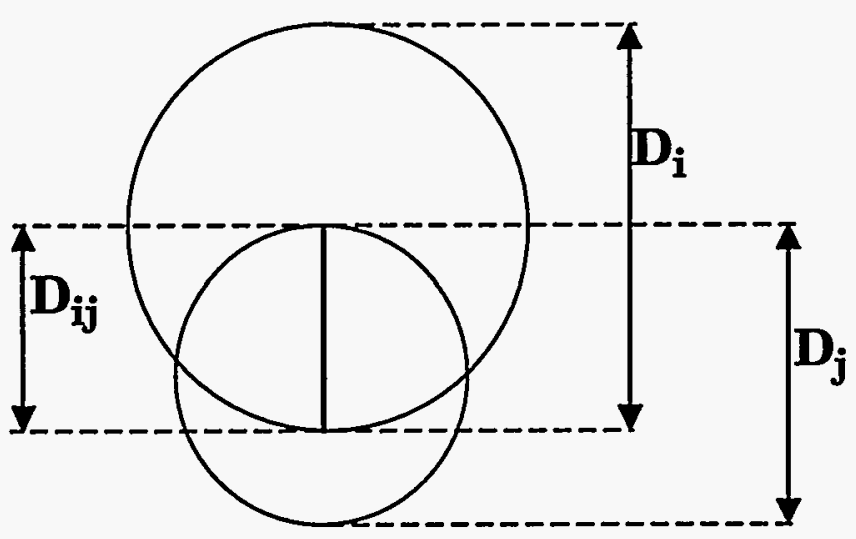

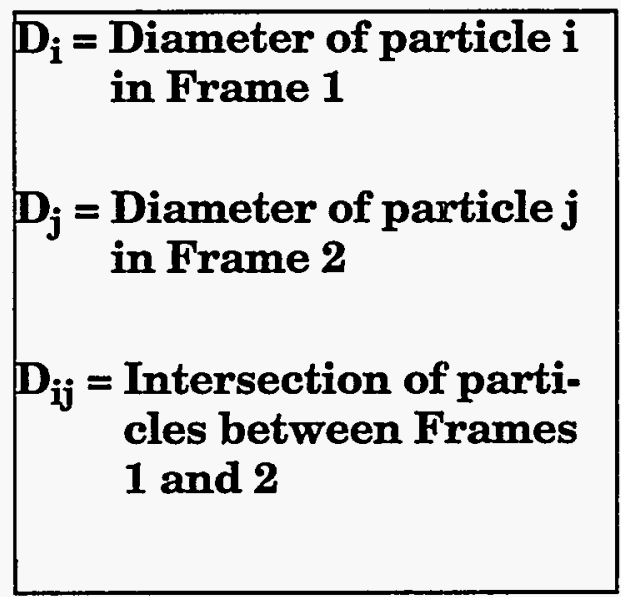

Figure 2.2. Intersection of particle images $i$ and $j$ between frames 1 and 2.

The possible pair with the largest $C_{i j}$ value, the largest $R_{i j}$ value, and the smallest sum of the distances between centroids is generally found to be the correct match. The velocity for a particle is then determined by dividing the particle movement by the known time between frames.

The Appendices provide step-by-step instructions (Appendix 1) and detailed descriptions of the programs (Appendix 2).

\subsubsection{Multi-Frame Tracking Algorithm}

The purpose of the Multi-Frame Tracking (MFT) algorithm is to track each particle through multiple time steps. This technique requires a minimum of four sequential images. A direct point-bypoint matching of particles from one image to the next is performed. The MFT code outputs the best particle tracks through all the sequential space images.

The sequential images contain the information necessary for the two-dimensional tracking scheme. The maximum number of images that can be tracked through at one time is limited only by the memory of the computer on which the code is executed. The MFT routine tracks particles through four frames directly, and then it combines the four frame tracks to indirectly track through all the frames available. Several possible tracks exist for each particle in the first interrogation area. Possible tracks are evaluated based on the variance of length and angle from a path of constant curvature, referred to here as the sigma value of the possible track. The possible track with the lowest sigma value is assumed to be the best track for that particle. Changes in size and gray level are measured between frames and can also be used to evaluate possible tracks. The tracking is accomplished by prediction of the displacement and direction of the particle through the four consecutive time steps. Figure 2.3 shows the tracking procedure in two dimensions. The search area in the sec- 


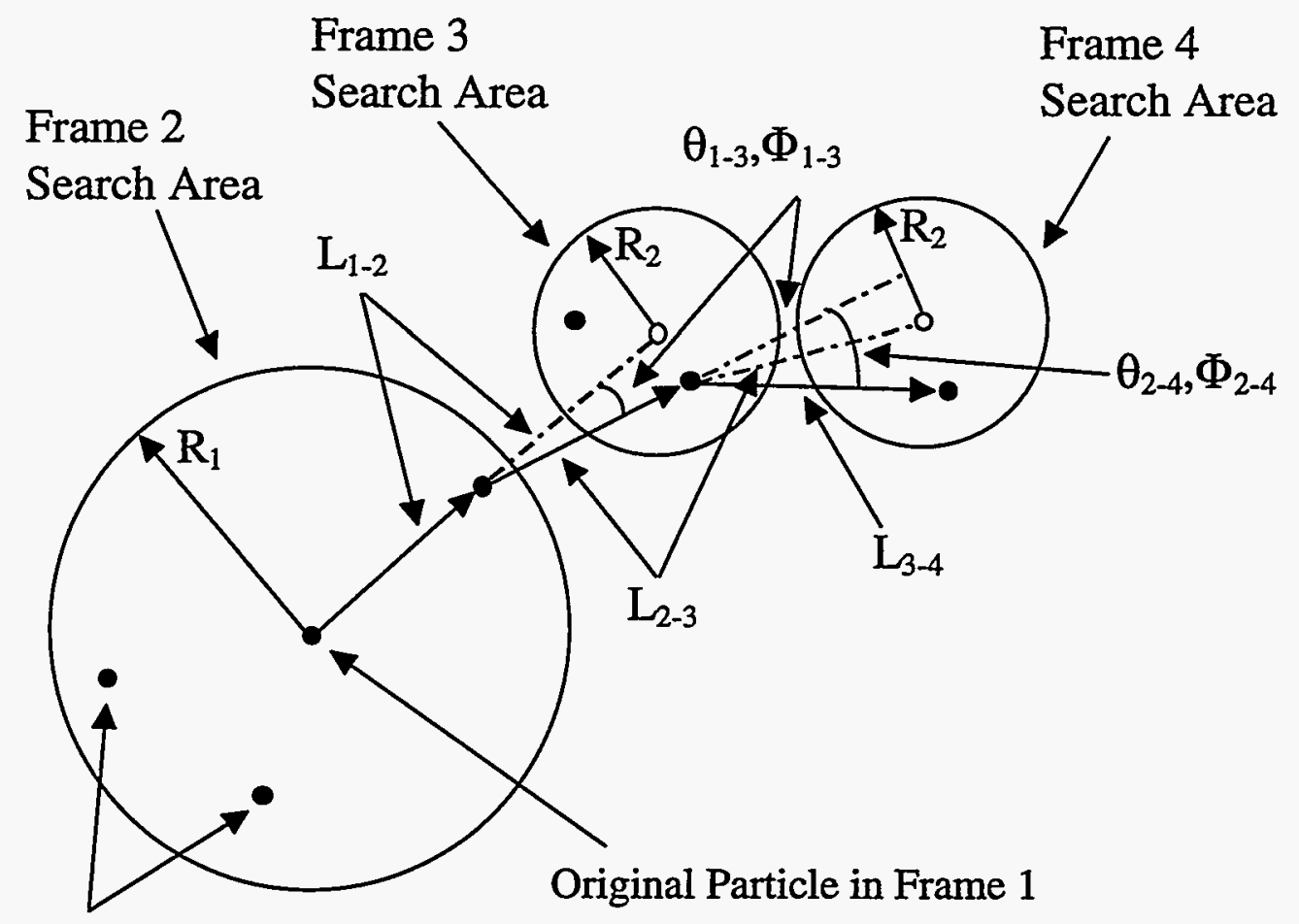

Candidate Particles in Frame 2

Figure 2.3. Determination of possible track for Multiframe Tracking (MFT) technique.

ond frame for a particle starting in the first frame is a circle of radius, $R_{1}$, determined by a rough estimate of the maximum velocity of the flow field. The search areas in the third and fourth frames have their radius, $R_{2}$, determined by a rough estimate of the maximum acceleration of the flow field. The center of each search area in Frame 3 is found by projecting out along the straight line, $\mathrm{L}_{1-2}$, of a possible track for each particle found in the search area of Frame 2. For each particle found in the search area of Frame 3, the center of a search area in Frame 4 is determined by using the length of the track from Frame 2 to $3, \mathrm{~L}_{2-3}$, and the angles created by the track from Frame 1 to 3, $\theta_{1-3}$ and $\Phi_{1-3}$. This results in several different possible tracks for each particle in Frame 1, the best of which is used as the actual track for that particle, as determined by the lowest sigma value. If different tracks originating in the same frame share a particle, then the one with the highest sigma value is discarded as incorrect. The following calculations are performed to determine the sigma value for each possible track:

$$
\begin{gathered}
\sigma_{1}^{2}=\frac{\left[\mathrm{L}_{1-2}-\overline{\mathrm{L}}\right]^{2}+\left[\mathrm{L}_{2-3}-\overline{\mathrm{L}}\right]^{2}+\left[\mathrm{L}_{3-4}-\overline{\mathrm{L}}\right]^{2}}{3} \\
\sigma_{\theta}^{2}=\frac{\left[\theta_{1-3}-\bar{\theta}\right]^{2}+\left[\theta_{2-4}-\bar{\theta}\right]^{2}}{2}
\end{gathered}
$$




$$
\begin{aligned}
& \sigma_{\Phi}^{2}=\frac{\left[\Phi_{1-3}-\bar{\Phi}\right]^{2}+\left[\Phi_{2-4}-\bar{\Phi}\right]^{2}}{2} \\
& \sigma^{2}=\sigma_{l}^{2}+\sigma_{\theta}^{2} \bar{l}^{2} \cos ^{2} \bar{\Phi}+\sigma_{\Phi}^{2} \bar{l}^{2} \sin ^{2} \bar{\Phi}
\end{aligned}
$$

Where:

$\sigma_{1} \quad$ Standard deviation from the mean for the lengths of the vectors

$\sigma_{\theta} \quad$ Standard deviation from the mean for the angles in the x-y plane

$\sigma_{\Phi}$ Standard deviation from the mean for the angles from the $\mathrm{z}$ axis (for 3D tracking, see Ch. 3)

$\sigma \quad$ Total standard deviation

$1_{1-2}$ Length between particles in the 1st and 2 nd frames

$\mathrm{l}_{2-3} \quad$ Length between particles in the $2 \mathrm{nd}$ and $3 \mathrm{rd}$ frames

$\mathrm{I}_{3-4} \quad$ Length between particles in the $3 \mathrm{rd}$ and 4 th frames

$\theta_{1-3} \quad$ Angle in the $x-y$ plane between $\mathrm{l}_{1-2}$ and $\mathrm{l}_{2-3}$

$\theta_{2-4} \quad$ Angle in the $x-y$ plane between $\mathrm{l}_{2-3}$ and $\mathrm{l}_{3-4}$

$\Phi_{1-3}$ Angle from the $\mathrm{z}$ axis between $\mathrm{l}_{1-2}$ and $\mathrm{l}_{2-3}$

$\Phi_{2-4}$ Angle from the $\mathrm{z}$ axis between $\mathrm{l}_{2-3}$ and $\mathrm{l}_{3-4}$ (for $3 \mathrm{D}$ tracking, see $\mathrm{Ch} .3$ )

i Average displacement of particles between frames $=\left(1_{1-2}+l_{2-3}+l_{3-4}\right) / 3$

$\bar{\theta} \quad$ Average $\theta$ of the track $=\left(\theta_{1-3}+\theta_{2-4}\right) / 2$

$\bar{\Phi} \quad$ Average $\Phi$ of the track $=\left(\Phi_{1-3}+\Phi_{2-4}\right) / 2$

The total standard deviation can be made a dimensionless value as follows:

$$
\sigma_{\text {total }}^{2}=\frac{\sigma^{2}}{\bar{l}^{2}}=\frac{\sigma_{l}^{2}}{\bar{l}^{2}}+\sigma_{\theta}^{2} \cos ^{2} \bar{\Phi}+\sigma_{\Phi}^{2} \sin ^{2} \bar{\Phi}
$$

Tracks can start in any frame of a multiframe sequence except the last three, so that each track can extend over four frames. After all four-frame tracks are found, any tracks that start in different frames but have the same particles in all their common frames are combined to form longer tracks, possibly spanning all the frames. For example, consider two four-frame tracks, the first through Frames 1-4 and the second through Frames 2-5. If the tracks share the same particles in frames 2, 3 , and 4, then they are combined into a single five-frame track. On the other hand, if the two fourframe tracks shared the same particles in frame 2, but had different particles in the other frames, then the track with the lowest sigma value would be kept, and the other track would be discarded.

The MFT algorithm is generally preferable to the CCT algorithm for multiphase flow measurements, where the separate phases can be tracked individually (Hassan and Blanchat, 1991a).

The Appendices provide step-by-step instructions (Appendix A) and detailed descriptions of the programs (Appendix B). 


\subsection{D PIV Validation Experiments}

This section presents two validation experiments that were performed using 2D PIV. The first is the flow over a square cavity, where both PIV and LDV were applied to the experiment and a computational simulation was also performed. The second flow is a thermal convection experiment in which PIV measurements were compared to a computational simulation.

\subsubsection{Cavity Flow Experiments}

The flow in a cavity provides a well-characterized separated, recirculating flow in a closed domain. There have been a number of previous experimental and computational examinations of the sheardriven flow in a cavity (e.g., Koseff and Street, 1984abc; Iwatsu et al., 1989), and this geometry is often used for computational model validation. However, most previous investigations have been concerned with the lid-driven cavity, in which the flow is driven by a belt which imposes a constant uniform velocity at the cavity upper surface. The cavity flow in the present study is driven by the laminar channel flow passing over the top of the cavity (cf. Reiman and Sabersky, 1968). This configuration is challenging for the PIV technique since it extends the required velocity range: the recirculating flow in the cavity has a peak velocity several times lower than that of the driving channel. Previous studies of the lid-driven cavity have shown complex three-dimensional effects, although many of those studies were performed at Reynolds numbers (Re) in the range 3000-10000, much higher than those considered here $(100<\operatorname{Re}<2758)$. For Reynolds numbers greater than approximately 3000, the flow becomes unsteady (Koseff and Street, 1984abc). Thus, the flows examined in this study are all expected to be steady, except possibly for the highest case $(\operatorname{Re}=2758)$. Laser Doppler velocimetry (LDV) was applied to measure point velocities throughout the cavity and channel, and PIV was then applied to measure full-field velocity maps in the same region.

\subsubsection{Experimental Setup}

There are a number of reasons that the cavity flow was chosen for this benchmark experiment. The extent of the recirculating region is independent of Reynolds number, thus easing the PIV imaging requirements. The flow is interesting because unusual three-dimensional recirculating regions are expected, although the present experiments are limited to examination of the two-dimensional centerline symmetry plane. Finally, there are a number of computational simulations of this flow for comparison, at least for the idealized case in which a fixed velocity condition is imposed at the cavity upper boundary.

The cavity flow geometry is set up in a small recirculating water tunnel (Fig. 2.4). The flow is pressure-driven, with the driving pressure supplied by a constant-head tank ( 62 by $62 \mathrm{~cm}$ cross-section, $114.5 \mathrm{~cm}$ deep). The water from the tank passes through a flow conditioning section consisting of a $62 \mathrm{~cm}$ long PVC pipe ( $9 \mathrm{~cm}$ I.D.) containing a packed bed of $3 \mathrm{~mm}$ glass beads. A 14 mesh stainless steel screen is placed at the downstream end of the flow conditioning tube. The flow passes through the tube into the 7.76 by $1.56 \mathrm{~cm}$ rectangular duct leading to the cavity. The cavity upstream corner lies approximately $193 \mathrm{~cm}\left(76^{\prime \prime}\right)$ downstream of the head tank (74 hydraulic diame- 


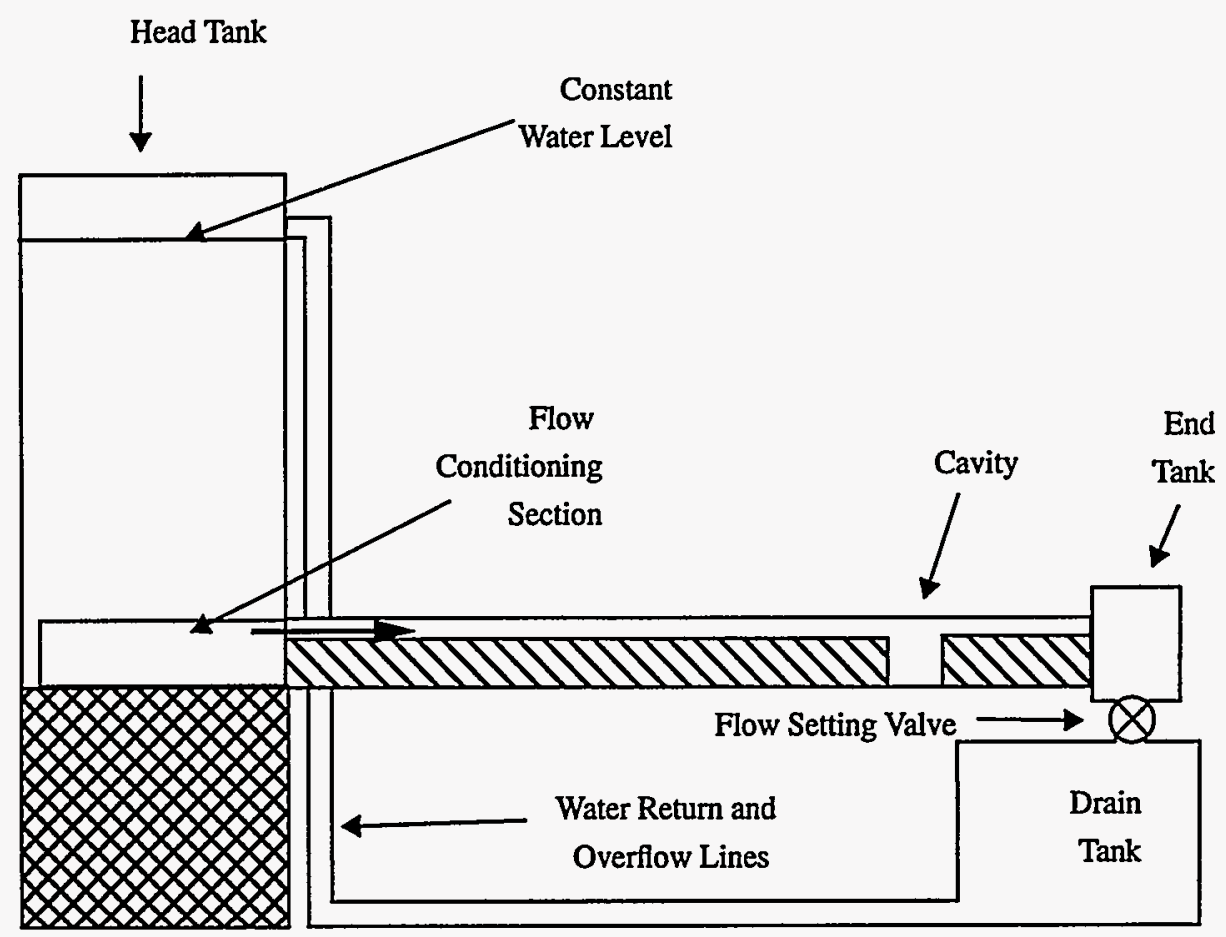

Figure 2.4. Schematic diagram of water channel/cavity flow.

ters). The downstream continuation of the channel, after the cavity, extends an additional $47.8 \mathrm{~cm}$ (18.8") before exhausting into an end tank. The water flows from the end tank to a drain tank through the flowrate-controlling valve. The water from the drain tank is then pumped back to the head tank, where a weir-type overflow connection back to the drain tank is used to maintain a constant head level. All walls are made of plexiglass to provide optical access.

Figure 2.5 is a schematic layout of the cavity flow geometry. A Reynolds number for this flow can be defined based on the mean incoming velocity $\bar{u}$ and the upstream hydraulic diameter, which for an infinitely wide channel is simply equal to twice the flow channel height $h$, yielding

$$
\operatorname{Re}=\overline{\mathrm{u}}(2 \mathrm{~h}) / \mathrm{v}
$$

where $v$ is the kinematic viscosity of the water. Although the channel here is not infinitely wide, this Reynolds number definition was chosen to allow comparison with 2D computations. The cavity depth and streamwise length are approximately 2.36 times the upstream channel height $(3.36: 1$ expansion), so Reynolds numbers based on the cavity depth or length are approximately $18 \%$ higher than the Reynolds number defined above. 

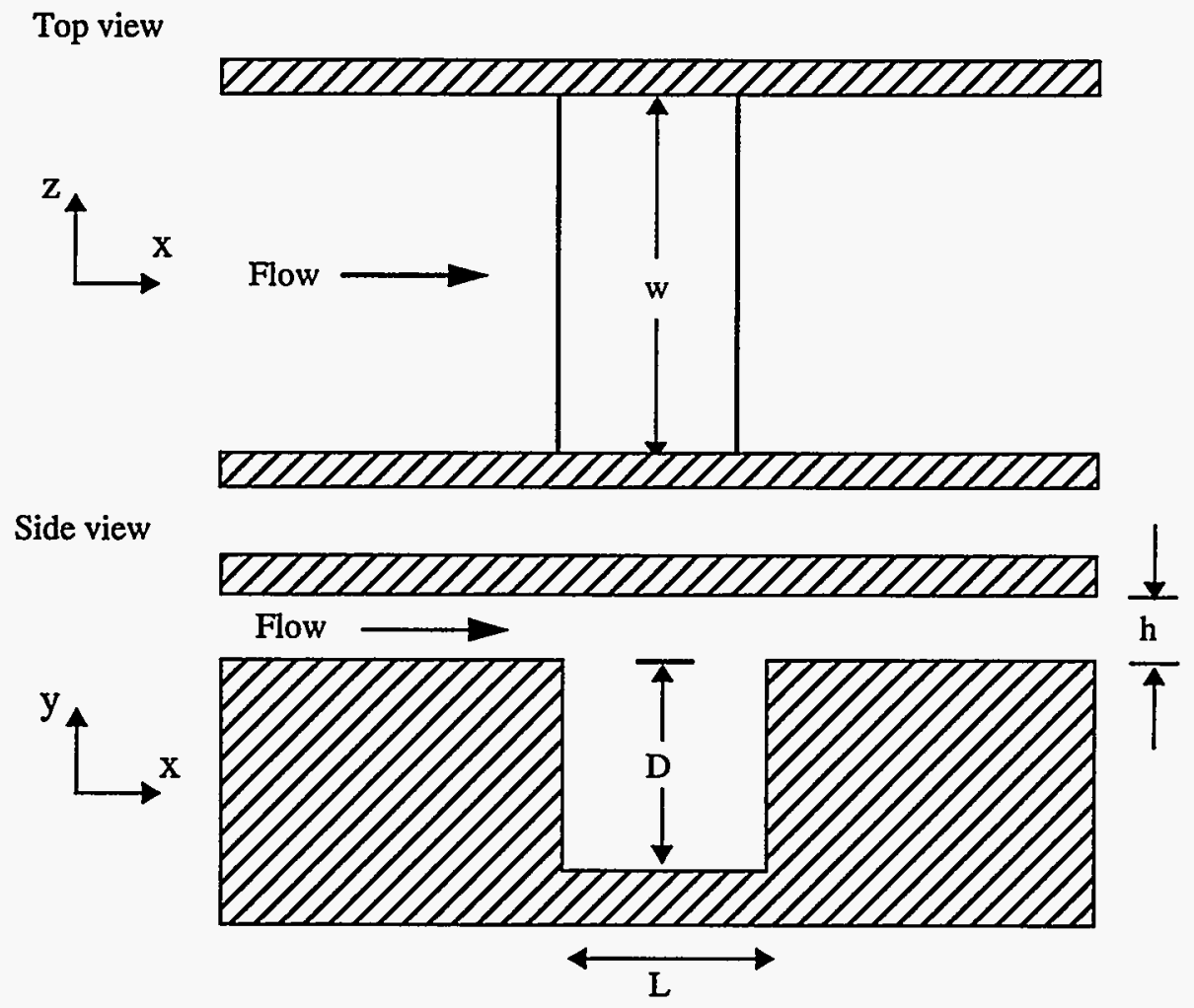

Figure 2.5. Schematic layout of cavity flow system. $D=3.68 \mathrm{~cm}, \mathrm{~L}=3.70 \mathrm{~cm}, \mathrm{~h}=1.56 \mathrm{~cm}$, $\mathrm{w}=7.76 \mathrm{~cm}$.

\subsubsection{Experimental Techniques}

\subsection{Laser Doppler Velocimetry (LDV)}

The LDV system used in this study is a two-component TSI (St. Paul, MN) fiber-optic probe system using a $5 \mathrm{~W}$ argon ion laser (Spectra-Physics Model 2020). The green $(514.5 \mathrm{~nm}$ ) and blue $(488 \mathrm{~nm})$ lines of the Art laser are used for measurement of the streamwise $(\mathrm{x})$ and vertical crossstream (y) velocity components, respectively. Frequency shifting is used on both channels. Data acquisition and analysis are performed using Model 1980B counter type processors, a Model MI990 data interface, and the TSI FIND software (Ver. 4.03) running on a 486-based computer.

The fiber-optic probe is positioned by a computer-controlled traverse. The light collection optics operate in the back-scatter mode. A $121.1 \mathrm{~mm}$ focal length probe lens $\left(10.78^{\circ}\right.$ half angle $)$ is used to focus the four laser beams at the measurement volume and to collect the Doppler-shifted light scattered by particles passing through the measurement volume.

Tap water, passed through a $10 \mu \mathrm{m}$ filter, is used to fill the tank. Since the fiber-optic LDV probe operates in the backscatter mode, it is necessary to add seed particles to the flow. Titanium dioxide 
$\left(\mathrm{TiO}_{2}\right)$ powder, ultrasonically dispersed in water, is added to provide seed particles. The $\mathrm{TiO}_{2}$ mean particle diameter is approximately $2 \mu \mathrm{m}$, and its specific gravity is approximately 4 . The $\mathrm{TiO}_{2}$ suspension was added until the water was slightly cloudy and the LDV data rate was approximately $1000 \mathrm{~Hz}$. Although $\mathrm{TiO}_{2}$ particles are too heavy to follow the flow with perfect fidelity, their small size gives them a very low settling velocity and allows them to remain suspended and to follow all but high frequency flow fluctuations (Durst et al., 1981).

\subsection{Particle Image Velocimetry (PIV)}

A CCD-based, particle-tracking PIV system was developed for this study, based on previous work by one of the authors (Blanchat, 1991; Hassan and Blanchat, 1991ab). In this setup, particle centroids are mapped out in each frame of an image sequence, then the particle images are paired up through the correlation algorithm (CCT and MFT) to determine particle displacements and velocities. The PIV system uses a $5 \mathrm{~W}$ argon ion laser for light sheet generation. The laser beam is shaped into a narrow line (approximately $1 \mathrm{~mm}$ thick) using conventional spherical and cylindrical lenses. The light sheet is directed downward through the flow from the top wall to the floor and spans the entire cavity width in the streamwise direction. Because of the continuous nature of the illuminating laser light sheet, care was taken not to heat the cavity floor. If the light sheet was left on for more than about $5 \mathrm{~s}$, localized floor heating created plainly visible regions of upwelling from the floor. For this reason, the light sheet is on for only one or two seconds during each PIV data acquisition period. Images are recorded with a CCD camera located perpendicular to the light sheet. The camera is a Pulnix Model TM745, with a 720 (x) by 480 (y) by 8 bit pixel format. Images can be recorded as quickly as every $1 / 30 \mathrm{~s}$ (33 ms apart). An image separation period of $1 / 30 \mathrm{~s}$ was used for all of the present experiments. Since the laser illumination for these experiments is continuous, the exposure period is determined by the camera shutter rate. The camera has an adjustable shutter, variable from $1 / 60$ to $1 / 10000 \mathrm{~s}$ exposure times. Exposure times of $1 / 60$ to $1 / 250 \mathrm{~s}$ are used for the present tests, which are sufficient because the flow velocities are low enough to prevent particle image blurring. The camera is connected to a frame grabber board (Epix 4MEG VIDEO Model 10 with 4 MByte memory), which is controlled by the Epix 4MIP frame grabber/image processing software running on a 486-based personal computer. This setup allows sequential acquisition of video images until the frame grabber memory buffer is filled. Eleven images are recorded in a typical flow sequence (approximately 3.8 MBytes).

Particle tracking from one image to the next has the advantage that there is no directional ambiguity, i.e., the direction as well as the magnitude of the velocity vector is directly measured. The video technology currently used limits data acquisition to approximately 30 frames/s. However, images of higher-speed flows could be made using two or more cameras or by double exposure of a single image with a pulsed light source.

The practical use of PIV requires the use of fast, reliable, computer-based methods for tracking the numerous particles suspended in a fluid flow. In some cases, image processing is performed on each frame prior to analysis by the PIV software. First, a background removal is performed by averaging together the eleven video frames, then subtracting the average frame from each of the other 
frames. Then the histogram of the pixel values in the image is plotted to determine the need for contrast enhancement. If the histogram shows the majority of the pixel intensities are at one end of the range (0 to 255), then image contrast enhancement is needed. Typically, most of the pixel values fall into the 0 to 100 range, so the contrast is enhanced by scaling all pixel values with intensities in the 0 to 100 range to the expanded 0 to 255 range, while all pixel values of 100 to 255 in the original image are set to 255 (white). After the contrast enhancement, a Laplacian filter is applied to sharpen the edges in the image. Finally, to increase the visibility of the bright particles in the image, all pixels below a determined threshold, usually 100 , are set to 0 (black). These steps convert a somewhat low-contrast original image to a sharp field containing well-defined particle images suitable for particle tracking with the PIV software.

Image data are analyzed in several steps. First, the particle centroid locations (gray level averaged), size, and average gray levels for each image recorded in a sequence are determined. Second, a dynamic, binary, spatial cross-correlation tracking method is used to analyze the PIV images. This method can be performed quickly if the particle tracer image information is first converted to binary data. That is, the 8-bit (0 to 255 gray level) pixel data defining a particle are converted to 2bit (value 0 or 1 ) pixels through image processing techniques, e.g., thresholding and connectivity algorithms. The particle velocity is found by determining the correspondence between particles in two sequential video frames. Correspondence is obtained using the CCT technique described in Section 2.2.1.

The PIV analyses were run on an Hewlett-Packard Model 720 RISC Workstation. Typical data processing times were approximately 1-2 hours for 1000 particle tracks in 10 image pairs.

An auto-cleaning validation method is used to eliminate manual operator assistance in identifying and removing erroneous vectors. This method is an iterative process involving an interpolated field produced from the most reliable vectors, which allows fast analysis and presentation of sets of PIV image data. The interpolation is based on the Hardy multiquadratic equations, and produces a vector at any given point in the flow field from the sparse, scattered velocity data (Blanchat, 1991).

\subsubsection{Experimental Approach}

LDV and PIV data were recorded at Reynolds numbers of $100,300,600,900$, and 2758. The experimental sequence was to make LDV scans one cavity depth upstream of the cavity entrance to check the incoming velocity profiles (in $y$ and $\mathrm{z}$ directions) and the flow steadiness. The velocity profiles were used to determine the mean velocity. When the desired Reynolds number was achieved, the flow was allowed to stabilize approximately one hour prior to data acquisition. At that time, the velocity profiles were checked, an LDV cavity scan was performed, followed by several sequences of PIV data acquisition, and finally a post-test LDV cavity scan and check of upstream velocity profiles to check flow steadiness. There were $458 \mathrm{LDV}$-velocity measurement locations within and above the cavity (see Figure 2.8). While the PIV images were captured in 1/60 to $1 / 250 \mathrm{~s}$, the LDV cavity scan data are time-averaged in the sense that each scan took approxi- 
mately two hours (1000 samples at each of 458 points). Both LDV and PIV data were recorded only on the flow centerplane.

\subsubsection{Computational Approach}

The computational fluid dynamics code FIDAP (Fluid Dynamics International, 1993) has been used to simulate the flow over and through the cavity-channel geometry. The flow is taken to be incompressible, isothermal, laminar, and steady, with constant material properties. The penalty method is used to enforce the incompressibility constraint, where a value of $10^{-5}$ is used for the penalty parameter. The experimental flow channel has a spanwise width of $7.763 \mathrm{~cm}$, and a height of $1.560 \mathrm{~cm}$, with a cavity in the lower side having a depth of $3.675 \mathrm{~cm}$ and a length of $3.700 \mathrm{~cm}$ (see Figs. 2.5 and 2.6). However, in these simulations, the following normalization is used: the den-

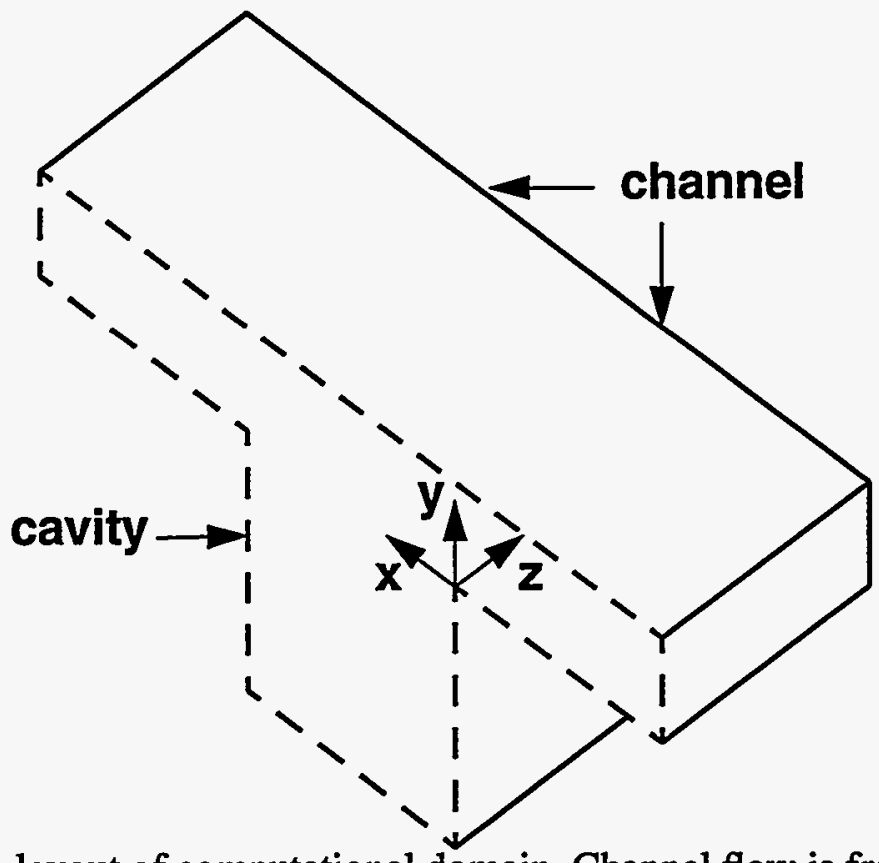

Figure 2.6. Schematic layout of computational domain. Channel flow is from lower right to upper left. Symmetry plane (dashed) is exposed to view.

sity $\rho$ and the channel mass-flow velocity $u_{a v}$ are set equal to unity, and the channel height $h$ is taken to be 0.5 . With this choice, the channel has a width of 2.488 , and the cavity has a depth of 1.178 and a length of 1.186 . The Reynolds number, here defined as $R e=\rho(2 h) u_{a v} / \mu=1 / \mu$, is thus specified by selecting a value for the viscosity $\mu$. A fully developed velocity profile with an average speed of unity is applied as the boundary condition at the inflow portion of the computational domain, natural boundary conditions (Fluid Dynamics International, 1993) are applied at the outflow boundary, symmetry conditions are applied on the channel midplane, and no-slip conditions are applied on all remaining surfaces (walls). The inflow and outflow boundaries in the computational domain are placed one cavity length upstream and downstream, respectively, from the cavity. Figure 2.6 shows a schematic diagram of the domain, and Figure 2.7 shows the two computational meshes computational meshes, composed of 27-node brick elements, on which solutions were obtained (Torczynski and O'Hern, 1994). Comparisons of results on these two meshes indicate that 

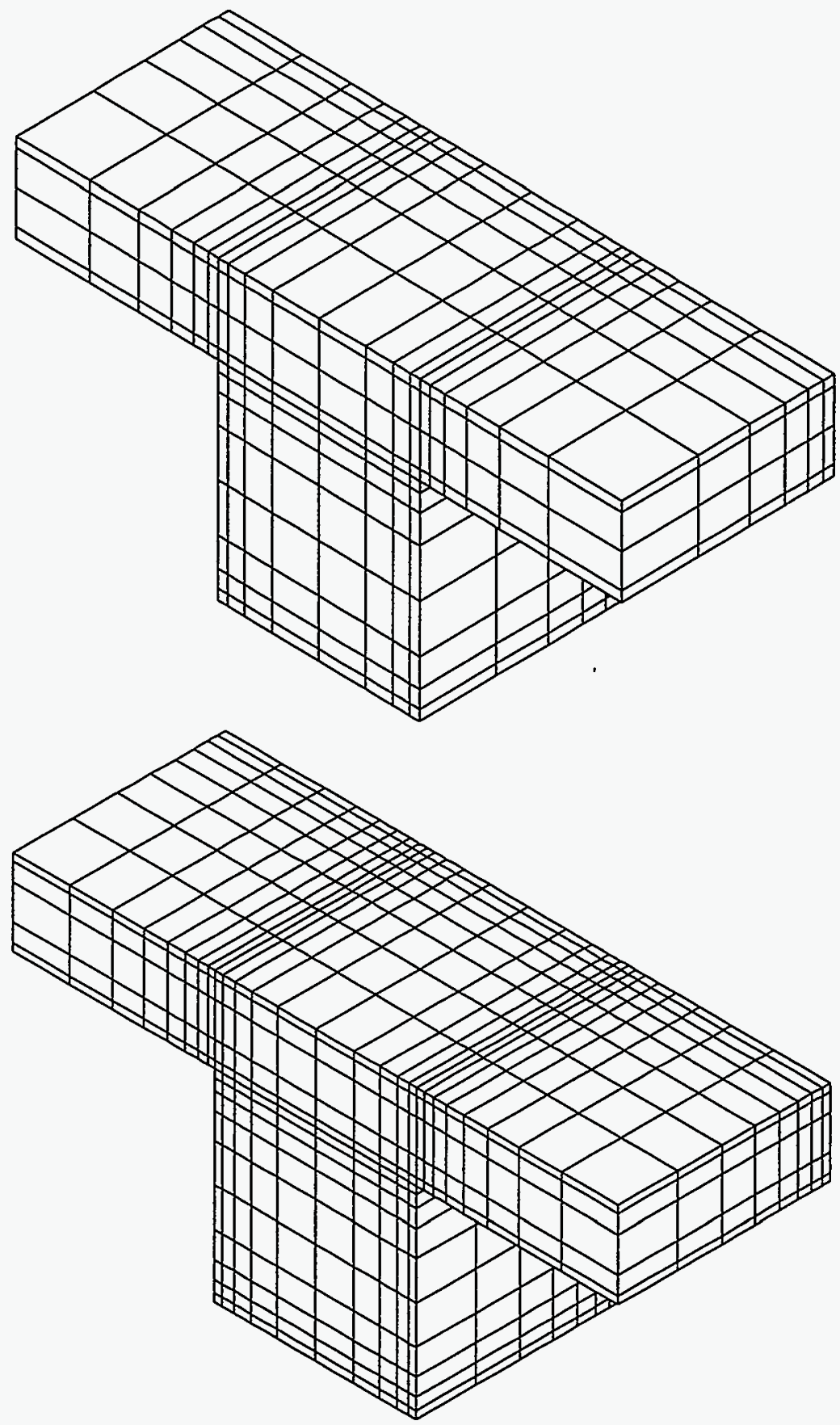

Figure 2.7. Computational meshes (27-node brick elements): top, coarse; bottom, refined. 
the second mesh has adequate resolution, so results are reported only for the second mesh. All postprocessing results are generated using the FIDAP post-processor FIPOST, which determines streamlines by integrating particle paths through the flow.

\subsubsection{Results of Cavity Flow Study}

\subsection{Laser Doppler Velocimetry (LDV)}

Figure 2.8 shows the LDV-measured planar centerline velocity fields recorded at $\operatorname{Re}=100,300$, 600,900 , and 2758. A primary recirculating region essentially fills the cavity. A small secondary recirculation region in the bottom upstream corner was evident for all Reynolds numbers examined. However, the LDV system was not well configured to examine the corner flows in much detail. The fairly short probe lens focal length and the fact that measurements were performed on the flow centerline led to beam clipping in the corner regions.

Typical LDV velocity measurements of laminar flows are known to have precision of about $\pm 1 \%$ or better (Durst et al., 1981). Measurement of the upstream peak velocity over 5 to 10 minute periods showed fluctuations on the order of $1 \%$ at $\operatorname{Re}=100$, and as discussed below, the upstream centerline velocity profiles (across both $\mathrm{y}$ and $\mathrm{z}$ directions) were typically constant to within the measurement accuracy over the several hour experiment run times.

Upstream velocity profiles were recorded at the start and end of each run at each of the Reynolds numbers of interest. Agreement was usually better than $1 \%$, with a maximum variation of $1.7 \%$ for the center velocity at $\operatorname{Re}=600$.

\subsection{Particle Image Velocimetry (PIV)}

Figure 2.9 shows the PIV-measured planar centerline velocity fields recorded at $R e=100,300$, 600,900 , and 2758. A primary recirculating region essentially fills the cavity. Again, the primary recirculation region essentially filled the cavity. At $R e=600$, secondary recirculation zones became visible in the two bottom corners of the cavity.

The raw PIV data consisted of velocity maps with a random distribution of vectors, depending on the location of trackable particles during data acquisition. The Hardy multiquadratic interpolation was used to calculate velocity vectors on a regular grid corresponding to the LDV measurement locations.

As expected, the cavity/channel flow combination was a demanding one in terms of the PIV velocity measurement range. The operator has to input the size of correlation window to search for particle pairs from one frame to the next, which can usually be determined by visual observation of the video images. This is typically done by visually locating the maximum displacement of particle images between frames (maximum velocity) and choosing a correlation window 1.5 to 2 times this length. However, this parameter varied significantly from the cavity region to the channel region, 

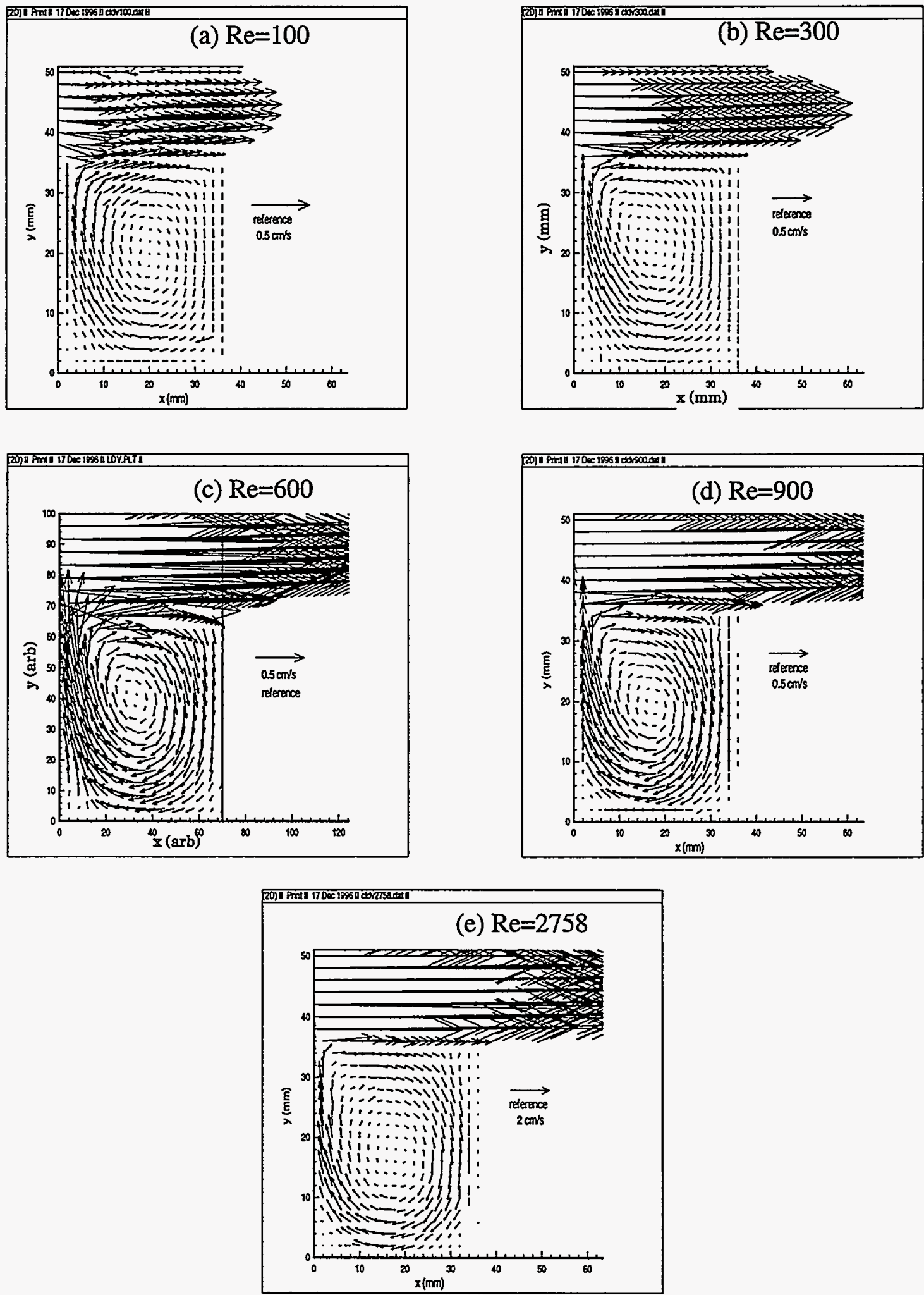

Figure 2.8. LDV results for cavity flow. Time-averaged centerplane velocity fields. 
(a) $\operatorname{Re}=100$

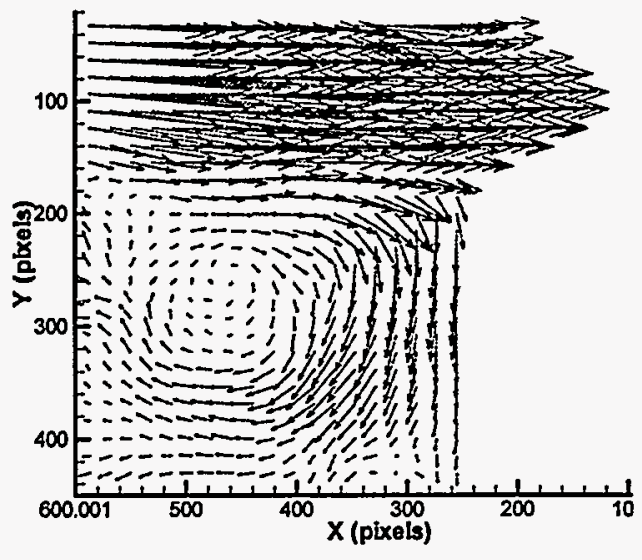

(c) $\mathrm{Re}=600$

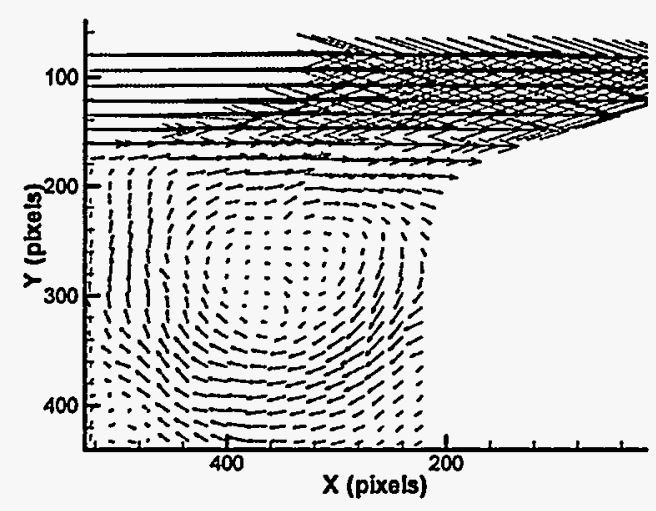

(b) $\mathrm{Re}=300$

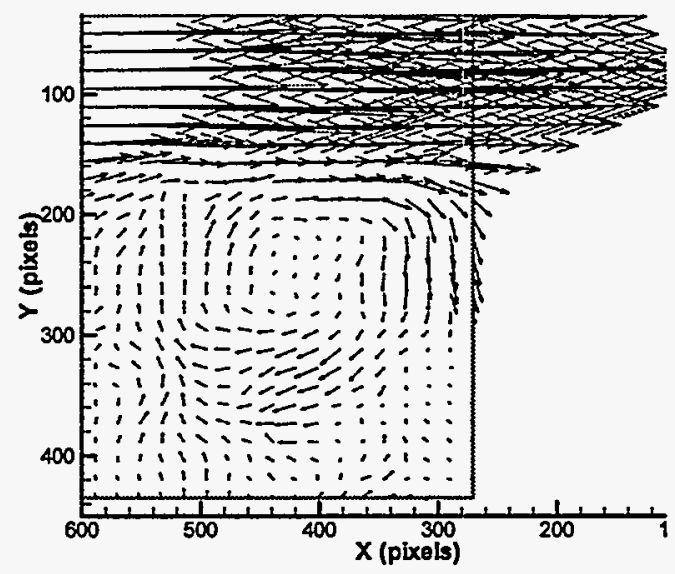

(d) $\operatorname{Re}=900$

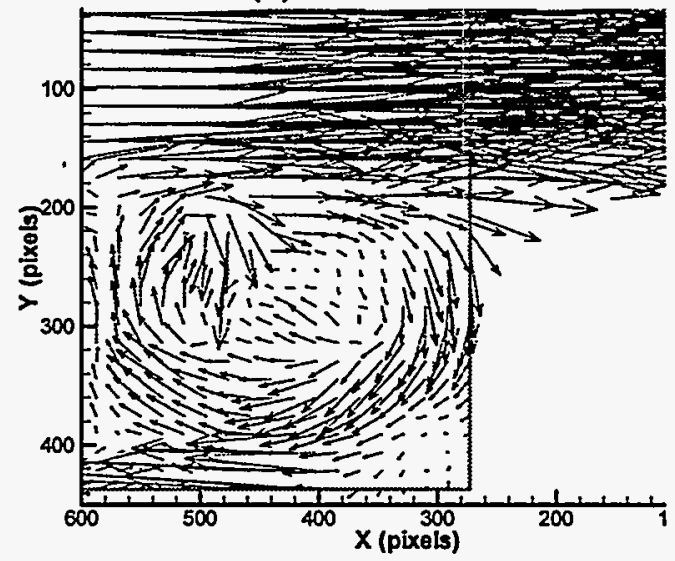

(e) $\operatorname{Re}=2758$

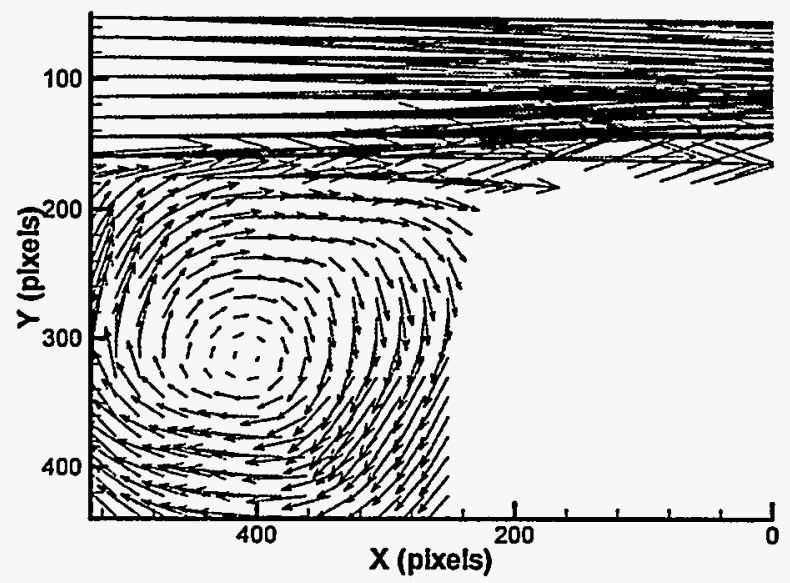

Figure 2.9. PIV results for cavity flow. Instantaneous centerplane velocity fields. 
so two values were used. This led to some problems at the cavity/channel interface, where it was not always clear where to stop using the larger window needed for the channel flow and start using the smaller window appropriate for the cavity flow. In addition, the video timing was optimized for the low-speed cavity flow rather than the higher-speed channel flow above the cavity. In general, the number of velocity vectors found by PIV in the channel was much lower than the number found in the cavity.

Sources of experimental error during PIV data recording include the timing between the video capture periods and the measurement of the cavity dimensions (for converting pixel locations to actual displacement). Both of these are thought to be better than $1 \%$. The main source of error in this technique is in the analysis, in particular in determination of which particles to match up for frame-toframe tracking. This can be complicated by out-of-plane motions (not expected on the present experiments performed on the centerline symmetry plane) and nonuniformities in the light sheet.

\subsection{Computational Simulations (FIDAP)}

Flow simulations are performed for Reynolds numbers of $1,3,6,10,30,60,100,300,600$, and 900. Figure 2.10 shows the centerplane velocity map, cf. Figs. 2.8 and 2.9 for LDV and PIV, respectively. Figure 2.11 shows how the location of the center of the main eddy varies with Reynolds number. Not evident in Figure 2.11 is the fact that the flow changes topology around $R e=27$. In the lower Reynolds number cases, some streamlines from the upstream section of the channel are subducted into the cavity, swirl about a streamline emanating from an inward spiral point while being displaced laterally away from the symmetry plane and toward the side walls, and ultimately exit from the cavity into the downstream section of the channel. In the higher Reynolds number cases, the subducted streamlines swirl about a closed isolated volume of fluid, rather than a single streamline. The fluid within this volume is kinematically similar to two opposed vortex rings being rotated about their axes by the shearing channel flow passing above the cavity: each half possesses a core streamline analogous to a vortex core through which the fluid flows toward the symmetry plane and outside of which the fluid flows away from the symmetry plane. Thus, the spiral point is outward here. Figure 2.12 shows streamlines for $R e=3$ and $R e=30$ illustrating these differences, and Figure 2.13 shows the topologies that correspond to these cases. See Torczynski and O'Hern (1994) for a more detailed discussion of these results. 


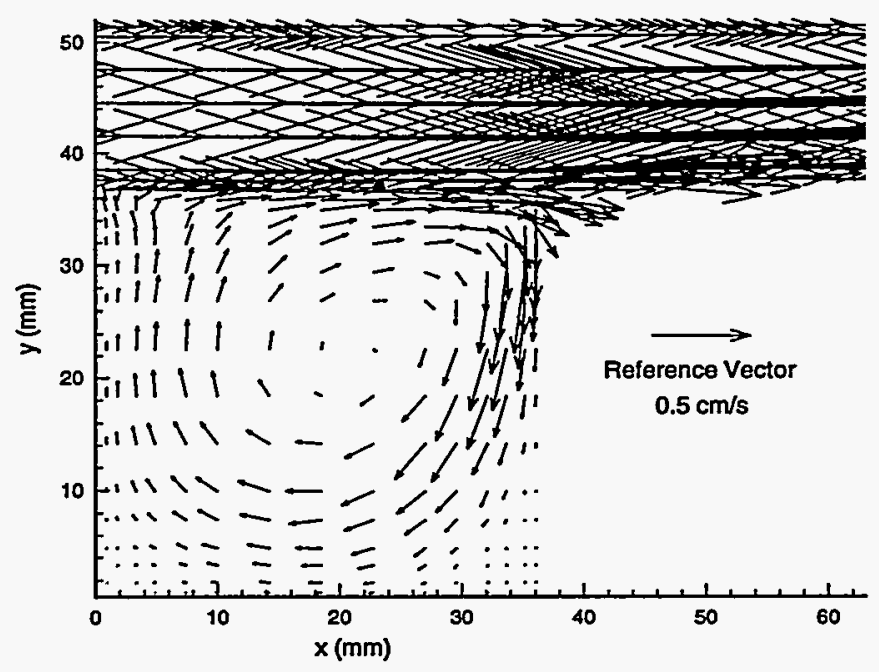

Figure 2.10. CFD simulations of velocity vectors in the cavity flow at $\operatorname{Re}=600$. Flow above the cavity is from left to right, with a peak velocity of about $2.8 \mathrm{~cm} / \mathrm{s}$.

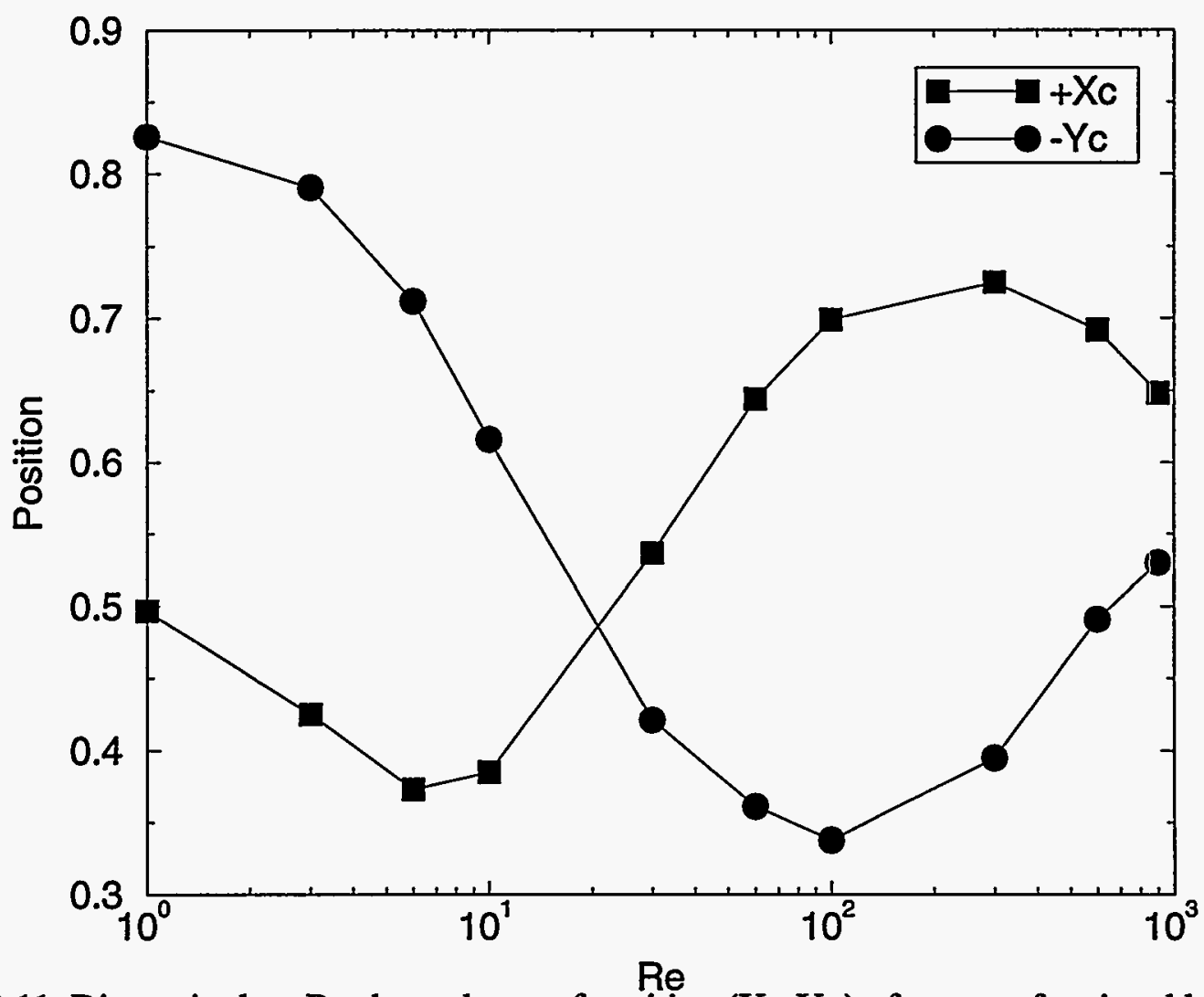

Figure 2.11. Dimensionless Re-dependence of position $\left(\mathrm{X}_{\mathrm{C}}, \mathrm{Y}_{\mathrm{C}}\right)$ of center of main eddy in the symmetry plane $\mathrm{z}=0$. 

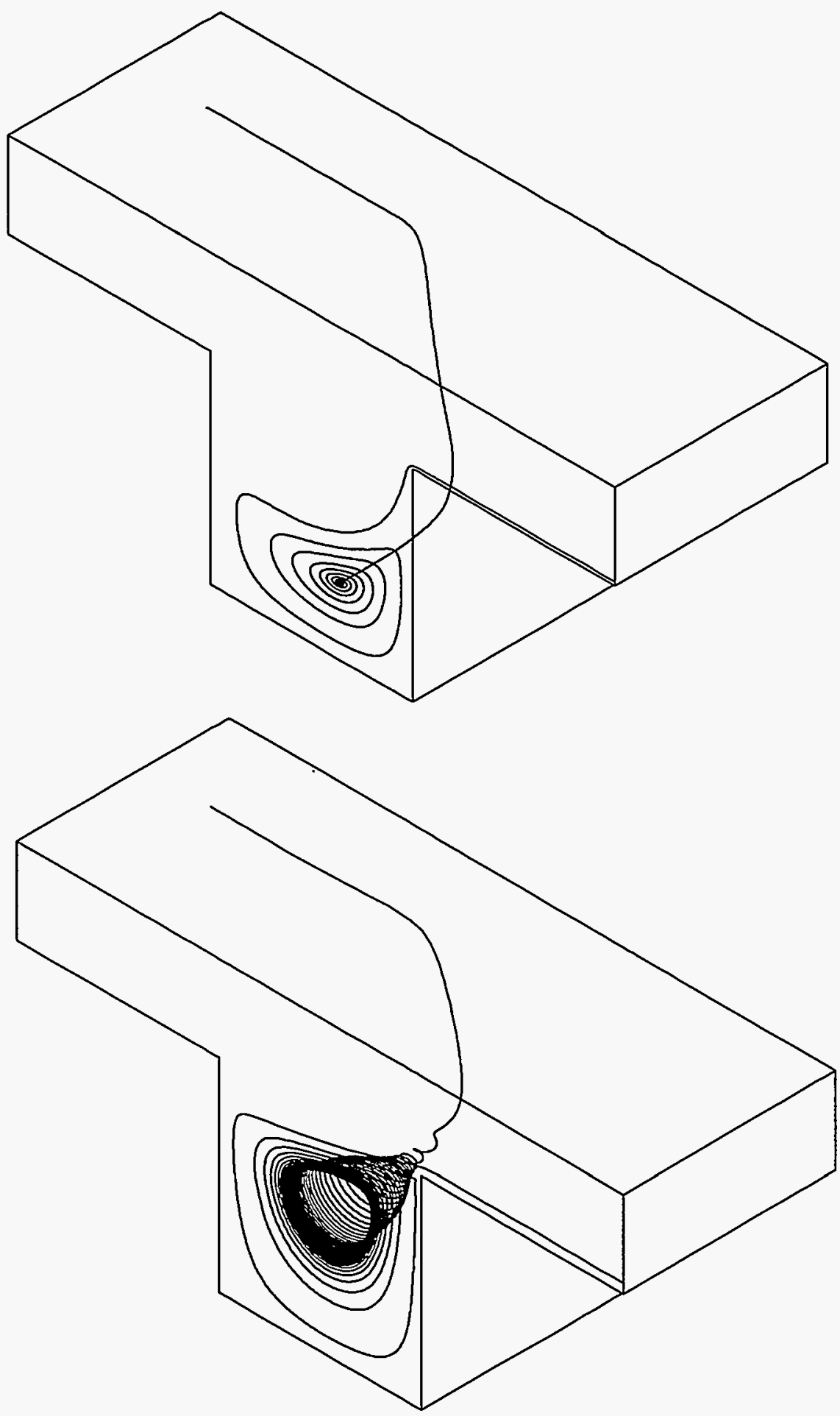

Figure 2.12. Streamlines: above, $\operatorname{Re}=3$ (no isolated region); below, $\operatorname{Re}=30$ (isolated region). 

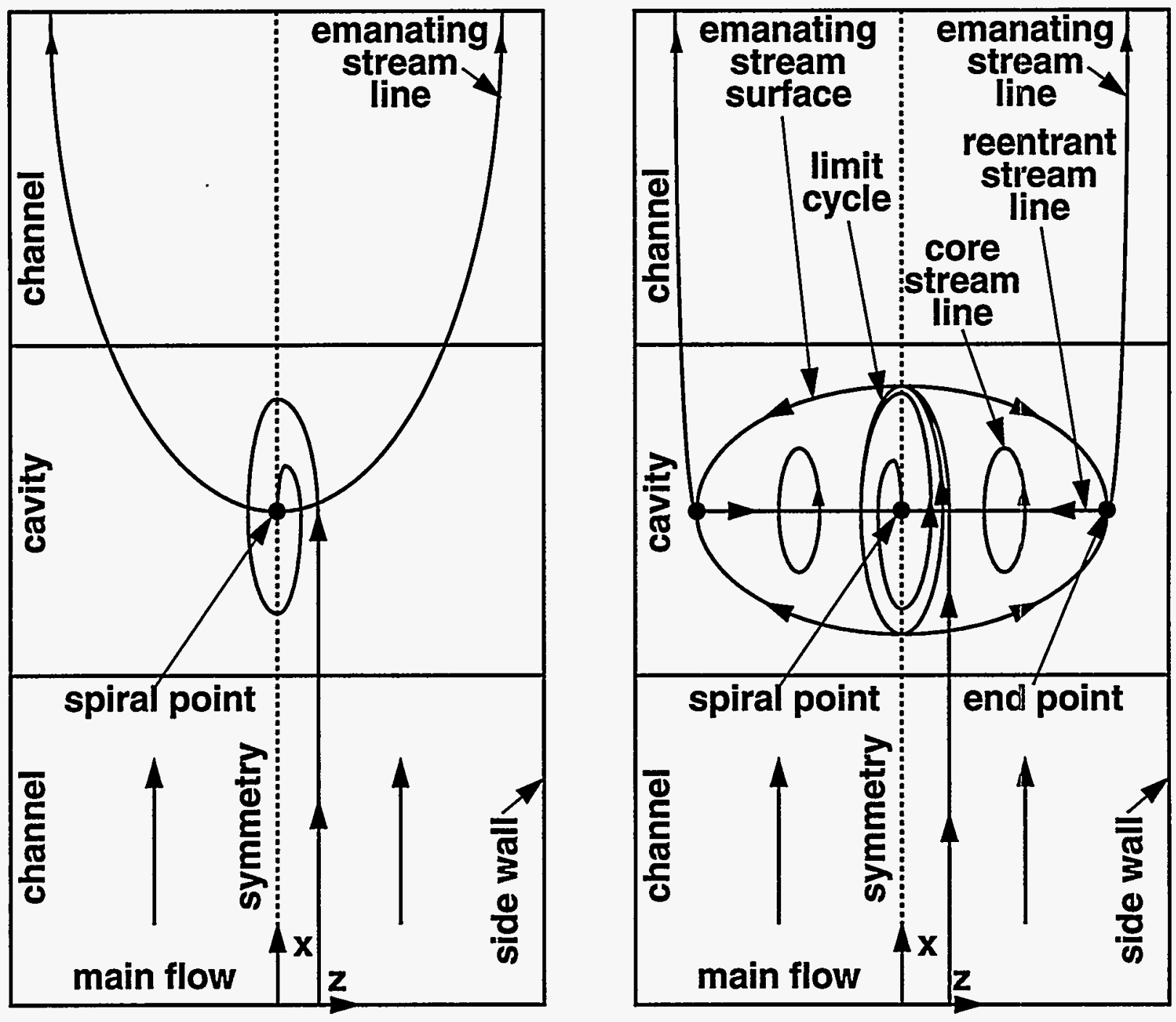

Figure 2.13. Topologies: left, low Re; right, high Re. Transition occurs at $\operatorname{Re}=27$. 


\subsection{Comparison of Results}

Examination of Figures 2.8, 2.9, and 2.10 show qualitative agreement between the LDV, PIV, and computed velocity fields, respectively, for $\operatorname{Re}=600$. Similar qualitative agreement was seen for $\operatorname{Re}=100,300$, and $900(\operatorname{Re}=2758$ was not computed). However, there are quantitative differences in terms of vortex center location and velocity vector magnitude. The reason for this discrepancy is not clear.

Quantitative comparison of the LDV and PIV data is made by examining the differences between the velocity vectors measured by the two techniques. This is done by subtracting the $\mathrm{u}$ - and $\mathrm{v}$ components of the PIV-measured velocity vectors from those measured by LDV, with the ideal difference being zero, of course. Although the channel flow above the cavity is qualitatively similar for LDV and PIV data (cf. Figs. 2.8 and 2.9), there were fairly large quantitative differences both in the channel and at the interface between the channel and the cavity. For $\operatorname{Re}=600$, the mean difference in the $u_{x}$ values above the cavity was $65 \%$. Figs. 2.8 and 2.9 show that the PIV-measured velocity was significantly lower. These differences were caused by the PIV auto-cleaning routine and the Hardy multiquadratic interpolation scheme. The auto-cleaning routine eliminates vectors based on particle pairs with a low correlation coefficient, which typically occur near walls, where there are few vectors (as mentioned above, few vectors were measured by PIV in the channel). The Hardy interpolation then uses a no-slip boundary condition at the walls which, when interpolated through the sparse near-wall velocity vectors, yields a smoothed velocity map containing lower than measured velocity vectors near the walls. Within the cavity, the agreement is better, although there is still a $10 \%$ difference in the average $u_{x}$ values measured by the two techniques. The largest discrepancies are near the walls and near the cavity/channel interface. At the other Reynolds numbers, much better agreement was found between the LDV and PIV mean u-velocity values above the cavity, with differences ranging from 2.9 to $4.2 \%$. Inside the cavity, however, the mean u-velocity difference was as high as $22 \%$ at $\operatorname{Re}=900$.

The $u_{y}$ comparison in the cavity at $\operatorname{Re}=600$ indicates generally better agreement. The mean $u_{y}$ in the cavity differs by $30 \%$ between the LDV and PIV data. The mean $u_{y}$ above the cavity is again $65 \%$ different. Most of the discrepancy in the cavity is caused by a large difference along the upper edge of the downstream cavity wall. For the other Reynolds numbers, the agreement is better, ranging from 7.8 to $24 \%$ difference in the cavity, and from 3 to $5 \%$ above the cavity. It is not clear why the $\operatorname{Re}=600$ case has the largest differences.

The difference between the LDV and PIV data is random, not systematic, except in the channel above the cavity, where the PIV velocities were always lower for the reasons discussed above. One probable reason for the random differences between the LDV and PIV data was that they were not run simultaneously since the same laser was used for both techniques. Even if they were started at the same time, the two techniques provide different data in that the LDV data is time-averaged over the run time (typically hours for 458 points in these runs), while the PIV is quasi-instantaneous (full data set of two frames in 1/30 s). The lack of true simultaneity is important because the LDV data recorded at the beginning and end of each run showed that, even in a time-averaged sense, there 
was some time variation in the flow. The main recirculating vortex center typically moved approximately $3 \mathrm{~mm}$ (about $9 \%$ of cavity) during the run. This can be seen by comparing LDV and PIV velocity maps (cf. Figs. 2.8 and 2.9), watching the PIV vortex center locations through several PIV sequences, or examining the LDV-measured turbulence intensity. The turbulence intensity (r.m.s. velocity fluctuations normalized by the local mean velocity) in the channel is typically one to two orders of magnitude lower than in the cavity, with the cavity peak occurring over a several millimeter region near the location of the vortex center. The source of this "unsteadiness" is unclear but is thought to be a problem in experimental conditions (e.g., room vibrations) rather than a true flow unsteadiness at these low Reynolds numbers. One possible source of the vortex movement is slight fluctuations in the head tank level (much less than 1\%). Since the vortex is moving, it is difficult to compare the near-instantaneous PIV-measured velocity field with the time-averaged LDV data (averaged both over data acquisition time and between the two LDV runs). In fact, even the PIV-determined vortex centers moved on the order of $1 \mathrm{~mm}$ from one sequence to the next, even though these data were recorded only a few seconds to minutes apart. Since the upstream LDV-measured velocity profiles were essentially constant, the source of this vortex movement is not clear. This vortex movement is considered to be the major source of disagreement between the LDV and PIV data and the computational simulations. Figure 2.14 shows LDV and PIV measured $u_{x}$ profiles through the vortex center for two of the five cases. The vortex center $x$ location was not the same between the LDV and PIV cases, but comparing the data in this fashion eliminates the effect of vortex movement. Figure 2.14 shows good agreement within the cavity for both cases, and even in the channel above the cavity for $\operatorname{Re}=100$. The comparison for the other three Reynolds numbers showed similar good agreement within the cavity, with fairly good agreement in the channel.

The computational results show a very interesting three-dimensional structure, with the nature of the recirculating region changing at a Reynolds number of approximately 27 . Unfortunately, the experiments were not well suited to examining such behavior, both because flow stability was difficult to achieve at these low Reynolds number and because the LDV and PIV experiments were run on $2 \mathrm{D}$ planes on the flow centerplane.

\subsubsection{Summary and Conclusions}

The shear-driven flow in a cavity was examined using two experimental techniques and computations. The flow in the cavity was driven by a fully developed laminar channel flow passing over the top of the cavity. The experimental techniques applied were laser Doppler velocimetry (LDV) and a video-based particle-tracking particle image velocimetry (PIV). The computational simulations were performed using the commercial finite element computational fluid dynamics (CFD) code FIDAP. The cavity Reynolds number ranged from 100 to 900 in the experiments, and from 0 to 1000 in the simulations.

Comparison of the experimental data indicates that the agreement between the LDV and PIV techniques is good through most of the flow but is poor near walls, especially in the channel above the cavity. This is partly due to the PIV auto-cleaning routine and the interpolation technique used 


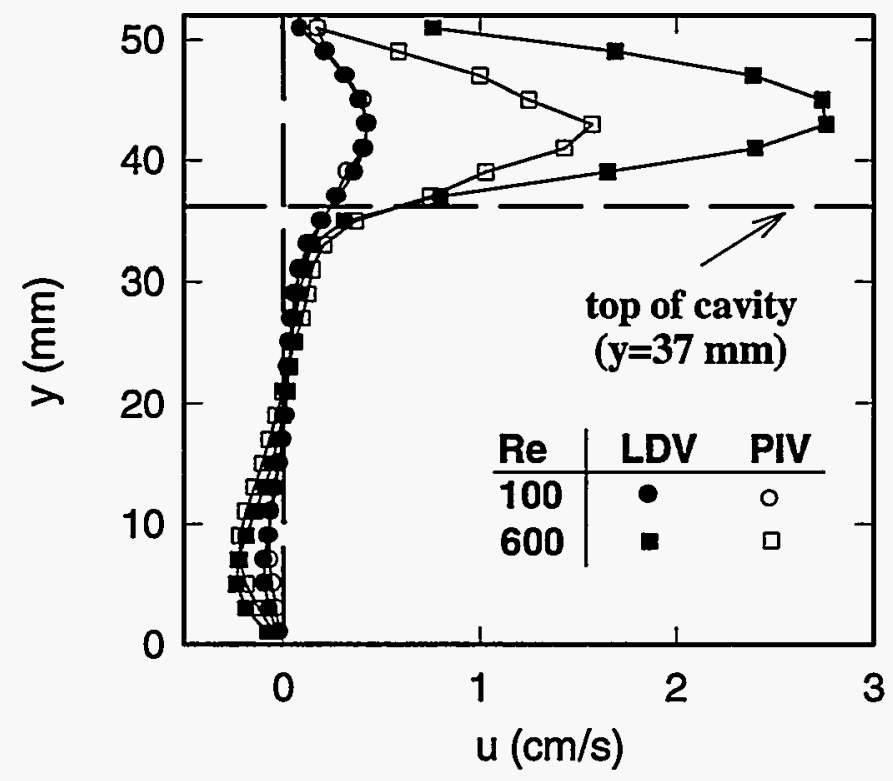

Figure 2.14. Comparison of LDV and PIV velocity profiles through the center of primary recirculation region in cavity, for $\operatorname{Re}=100$ and $\operatorname{Re}=600$.

to map the random PIV data onto a regular grid for comparison with LDV, partly due to optimization of the data acquisition for the lower speed cavity flow, and partly due to unexpected flow unsteadiness (vortex wandering). As expected, the cavity/channel flow combination was a demanding one in terms of the velocity measurement range of the PIV technique.

The computational simulations show an interesting three-dimensional structure in the recirculating region. The computed centerplane velocity map is in fair qualitative agreement with the experimental data. However, there are quantitative differences in terms of vortex center and velocity magnitude. The reasons for these discrepancies are still under investigation.

\subsubsection{D Thermal Convection Experiments}

The natural convection of a fluid in an enclosure is examined. This study is motivated by a possible magma-energy extraction system discussed by Chu et al. (1990), in which a well drilled through the Earth's crust penetrates the magma, and fluid circulation in the well keeps the rock adjacent to the penetration below the melting point (see Figure 2.15). Thus, the magma-energy extractor can be approximately described as a cold cylinder penetrating vertically downward into a hot fluid 


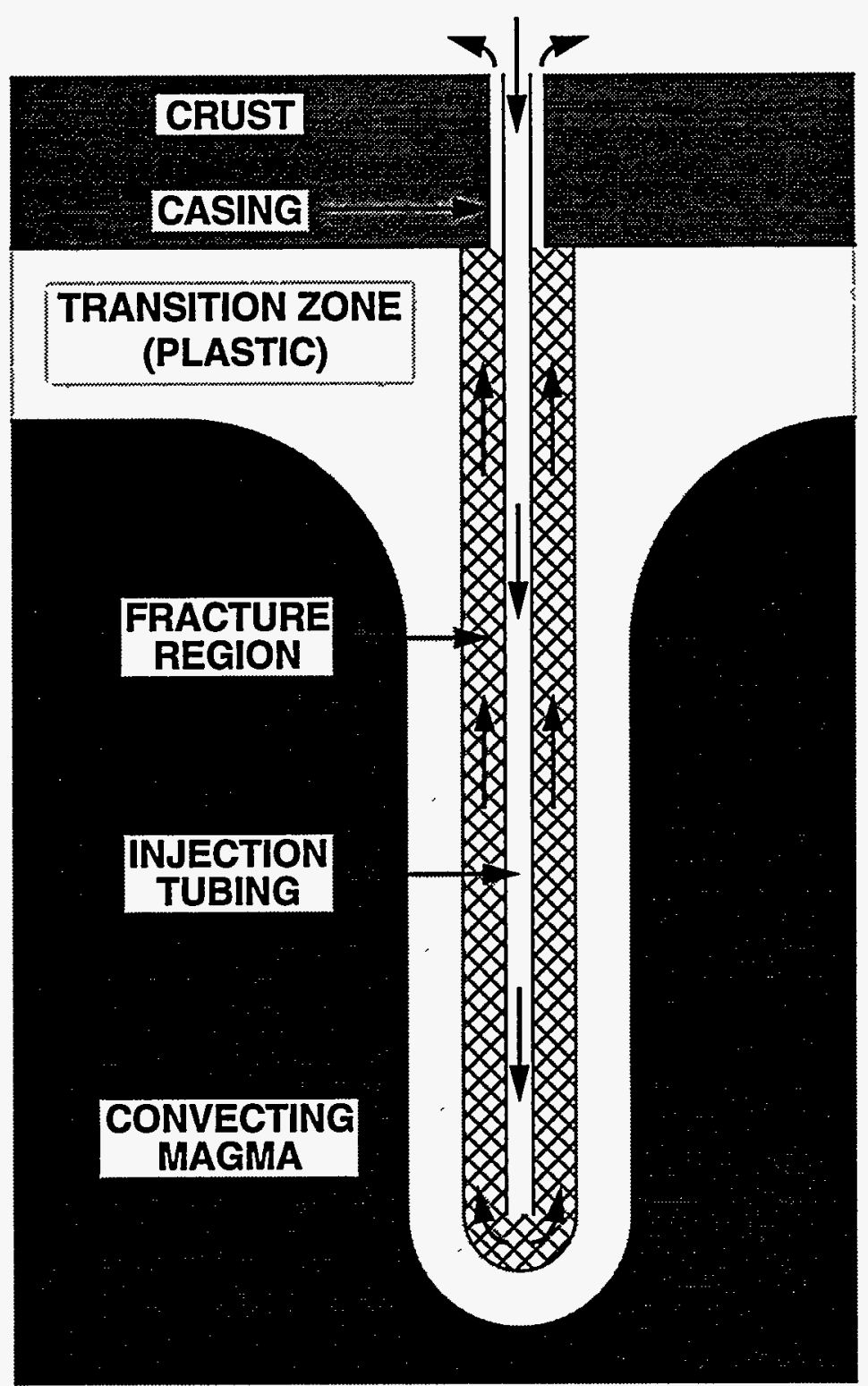

Figure 2.15. Conceptual representation of a single well of a magma-energy extraction system in operation (cf. Chu et al., 1990).

undergoing natural convection. An additional issue is that the viscosity of magma decreases strongly with increasing temperature (cf. Chu and Hickox, 1990; Hickox and Chu, 1991).

A schematic diagram of the experiment is shown in Figure 2.16. Buoyancy-driven convection is examined in a cube, where each side of the cube measures $56 \mathrm{~cm}$. The bottom of the fluid-filled volume is heated with a flush mounted $14 \mathrm{~cm}$ by $56 \mathrm{~cm}$ heater, centered on the bottom surface. The top of the volume is enclosed by a water-cooled constant-temperature plate. A $28-\mathrm{cm}$ long, $7.6 \mathrm{~cm}$ diameter cylinder, attached to the constant temperature plate, also water-cooled, protrudes into the 
fluid. The vertical sides of the cube are insulated. The working fluid is $42 / 43$ corn syrup. Because of the large viscosity variation associated with temperature, this fluid has been used in magma convection experiments (Chu and Hickox, 1990; Hickox and Chu, 1991). A three-dimensional convection flow is developed in the cubic volume as a result of the interaction between the cool downward-flowing boundary layer around the cylinder and the plume driven by the heated strip centered on the bottom surface. Because of the high viscosity of the working fluid, the flow remains laminar despite the large dimensions of the experiment, making it seem like an ideal test bed for three-dimensional PIV (see, however, Ch. 3). Glass balloons are used as tracer particles. As received, the size of the balloon ranges from 20 to $100 \mu \mathrm{m}$. The particles are sieved such that all particles are larger than $70 \mu \mathrm{m}$. Approximately $2 \mathrm{mg}\left(\approx 0.003 \mathrm{~cm}^{3}\right)$ of particles are added to each 20 liter syrup container, resulting in a particle density of approximately 1 particle/ $\mathrm{cm}^{3}$. The particles stay in suspension indefinitely.

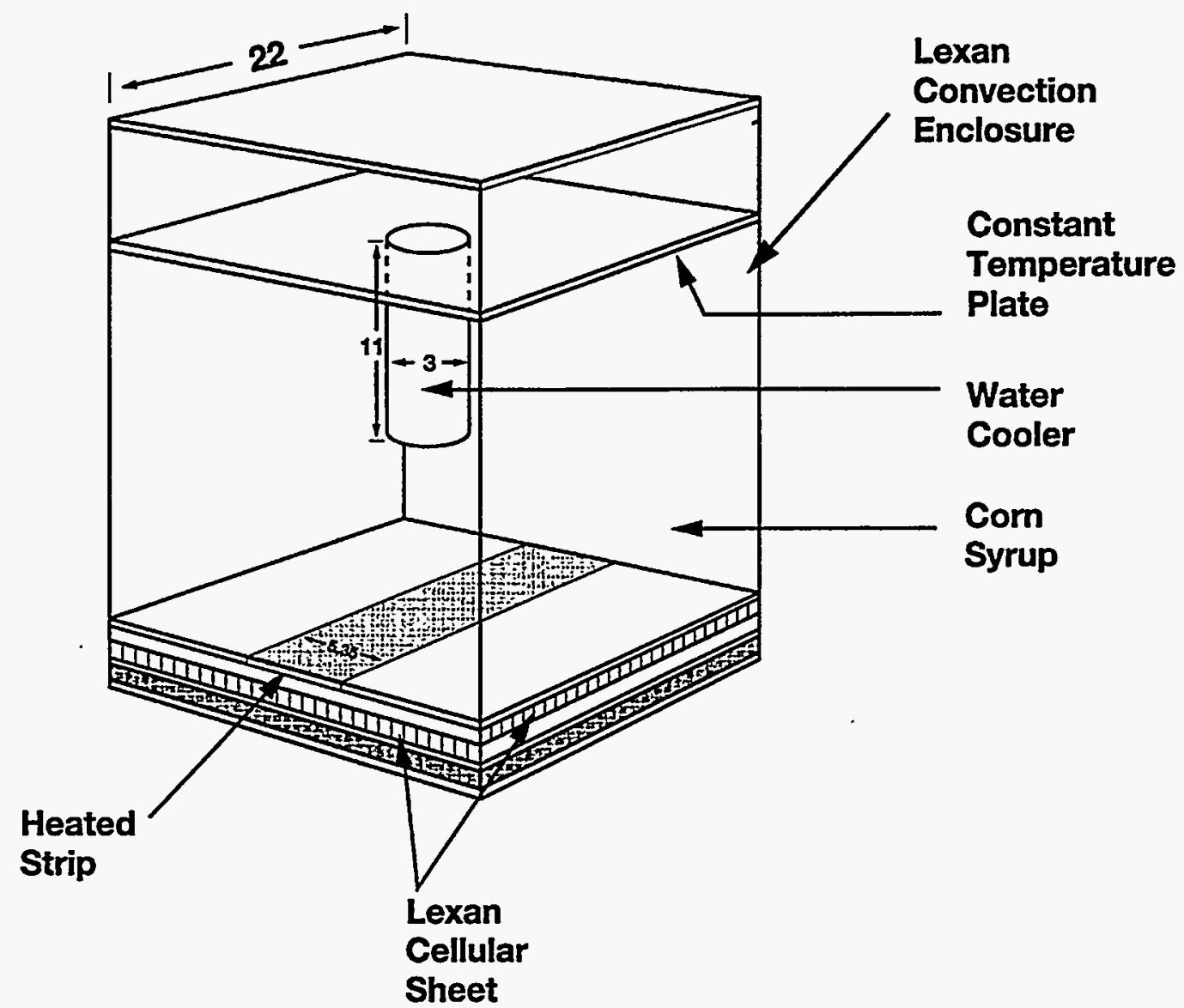

Figure 2.16. Experimental setup for thermal convection experiments. Dimensions are in inches.

The convection enclosure is an open top box with a square platform measuring $56 \mathrm{~cm}$ on the side and $60-\mathrm{cm}$-high and is constructed from 13-mm-thick Lexan (polycarbonate) sheets. The bottom of the box is a three-layer structure. The top layer is made of a sheet of 13-mm-thick Lexan with a 
14-cm-wide center recess machined into the sheet to accept the heated strip. This top layer is glued to two 10-mm-thick Lexan glazing sheets. The glazing sheets are extruded sheets with longitudinal cells, providing structural strength and effective insulation. The heated strip consists of a 3-mmthick copper plate with a thin, flexible, etched foil heater glued on the underside. The resistance of the foil heater is nominally $8 \Omega$. The temperature of the heated strip is monitored by 12 thermocouples embedded in the copper plate. In addition, there are 7 thermocouples in the unheated portion of the bottom plate. The side wall temperature is similarly monitored with 3 thermocouples. The thermocouples are made of $0.25 \mathrm{~mm}$ copper-constantan wires mounted flush with the surface.

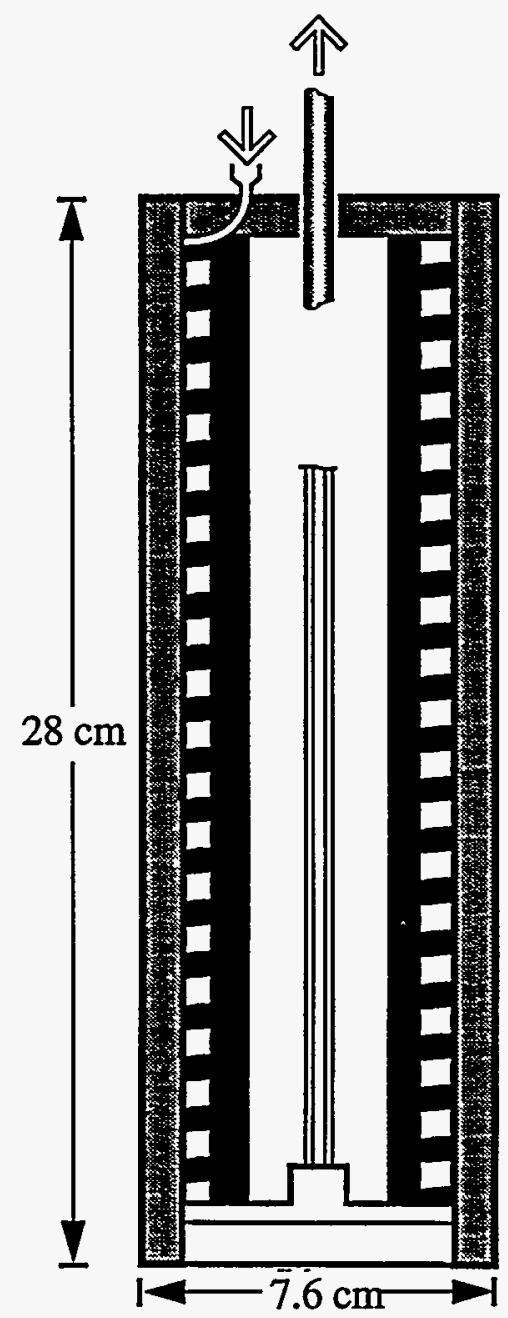

Figure 2.17. Schematic diagram of cooling cylinder inserted into convection tank.

The constant temperature plate bounding the fluid volume from above and the cylinder protruding into the fluid volume are both made of brass. The constant temperature plate is $25.4 \mathrm{~mm}$ thick. The temperature of the plate is maintained by the circulating cooling water, from a temperaturecontrolled bath (Neslab model HX-75), in twenty $13 \mathrm{~mm}$ by $19 \mathrm{~mm}$ parallel channels machined in 
the back of the plate. The back of the plate is sealed by a 6-mm-thick brass plate. The cooled cylinder is a three-piece construction. The side of the cylinder is made of two pieces, one sheathed over the other (see Figure 2.17). The inner piece is essentially a $25 \mathrm{~cm}$ long brass screw with 13$\mathrm{mm}$ by $13-\mathrm{mm}$ threads on a $25.4-\mathrm{mm}$ pitch. An outer piece, of 7.6-cm-diameter by $25.4-\mathrm{cm}$-long with $13 \mathrm{~mm}$ thick wall, slides over the inner piece to form a spiral cooling channel. The mating surfaces are slightly tapered to achieve a watertight construction. The spiral channel is connected to the bottom bulkhead consisting of concentric water channels. The plate and cylinder temperatures are monitored using Type E (Chromel-Constantan) thermocouples. During the experiment, the plate temperature was found to be uniform within $0.05^{\circ} \mathrm{C}$ for plate temperatures ranging from 15 to $50^{\circ} \mathrm{C}$ and within $0.1^{\circ} \mathrm{C}$ for plate temperatures below $6^{\circ} \mathrm{C}$. The electric power to the heater is determined to an accuracy of $0.2 \%$. The net rate of heat transfer to the surrounding is no more than $8 \%$ of the total electric input at steady state. The maximum total error in the determination of the Rayleigh number is $10.2 \%$. For the two-dimensional experiments the constant-temperature plate and cylinder were kept at $20^{\circ} \mathrm{C}$, and the heater was kept at $58^{\circ} \mathrm{C}$.

Flow Chamber

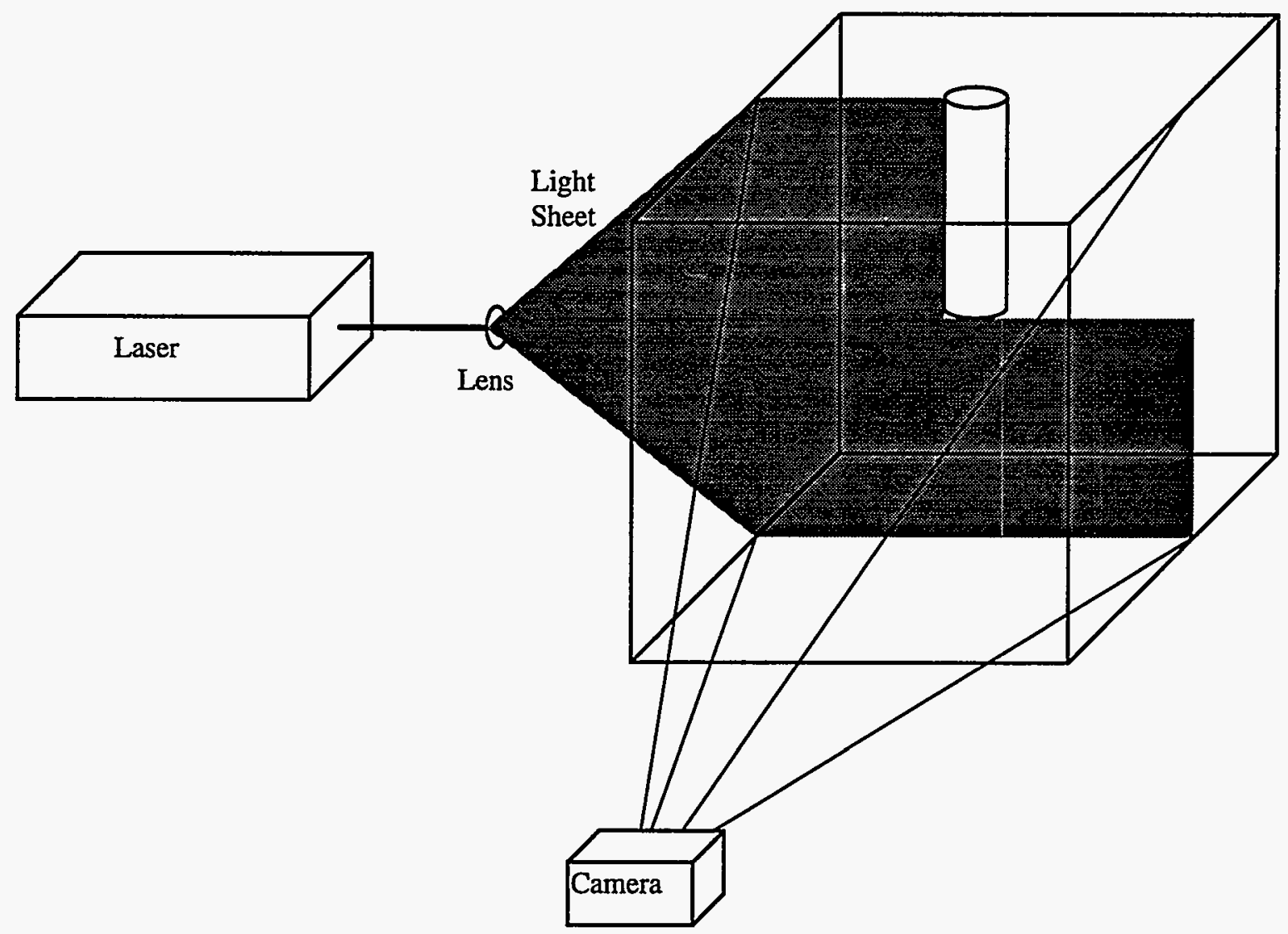

Figure 2.18. 2D PIV setup for thermal convection experiments.

Figure 2.18 shows the basic two-dimensional PIV set-up for this experiment. A light sheet was used to illuminate the particles in a plane for two-dimensional PIV. The light sheet was created by 
expanding collimated laser light through a cylindrical lens. A Spectra-Physics model 165 Argon ion laser was used to produce the light. Pictures of the illuminated particles were captured using a $35 \mathrm{~mm}$ film camera. These pictures were digitized using a Nikon Coolscan on a 486-based computer.

\subsubsection{Results}

The two-dimensional PIV routines worked fairly well for this experiment. Figures 2.19 shows flow-visualization photographs for the center planes intersecting and bisecting the heater strip. Figures 2.20 are PIV results for equivalent planes.

Figure 2.21 shows finite element CFD simulation of selected streamlines in the symmetry planes. Details of the computational techniques are given in Chapter 3. Figures 2.22 shows streamlines calculated from the PIV velocity fields given in Figures 2.20. Qualitative agreement of the streamlines between experiment and computation can be seen.

Unfortunately, the computational models were unable to converge at the high temperatures used in the experiment, so an exact comparison was impossible. However, the flow computed at lower temperatures is very similar to the flow shown by the PIV results at higher temperature. 

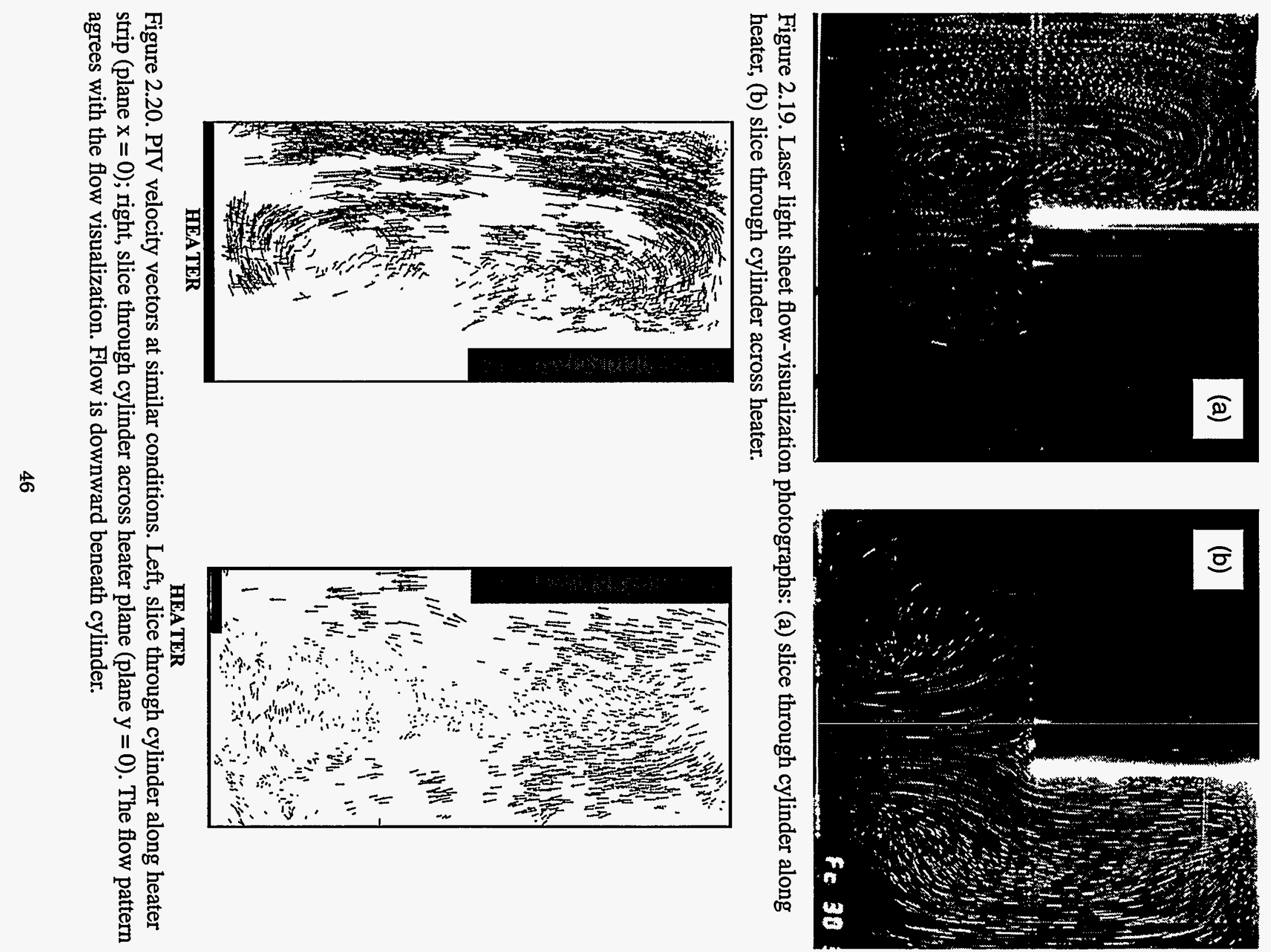


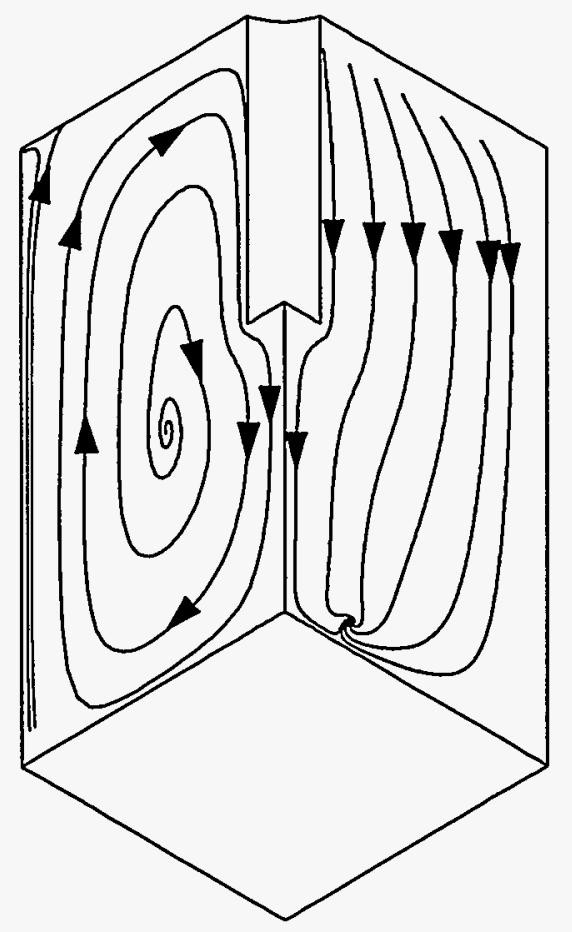

Figure 2.21. Finite element CFD simulation of selected streamlines in the symmetry planes.
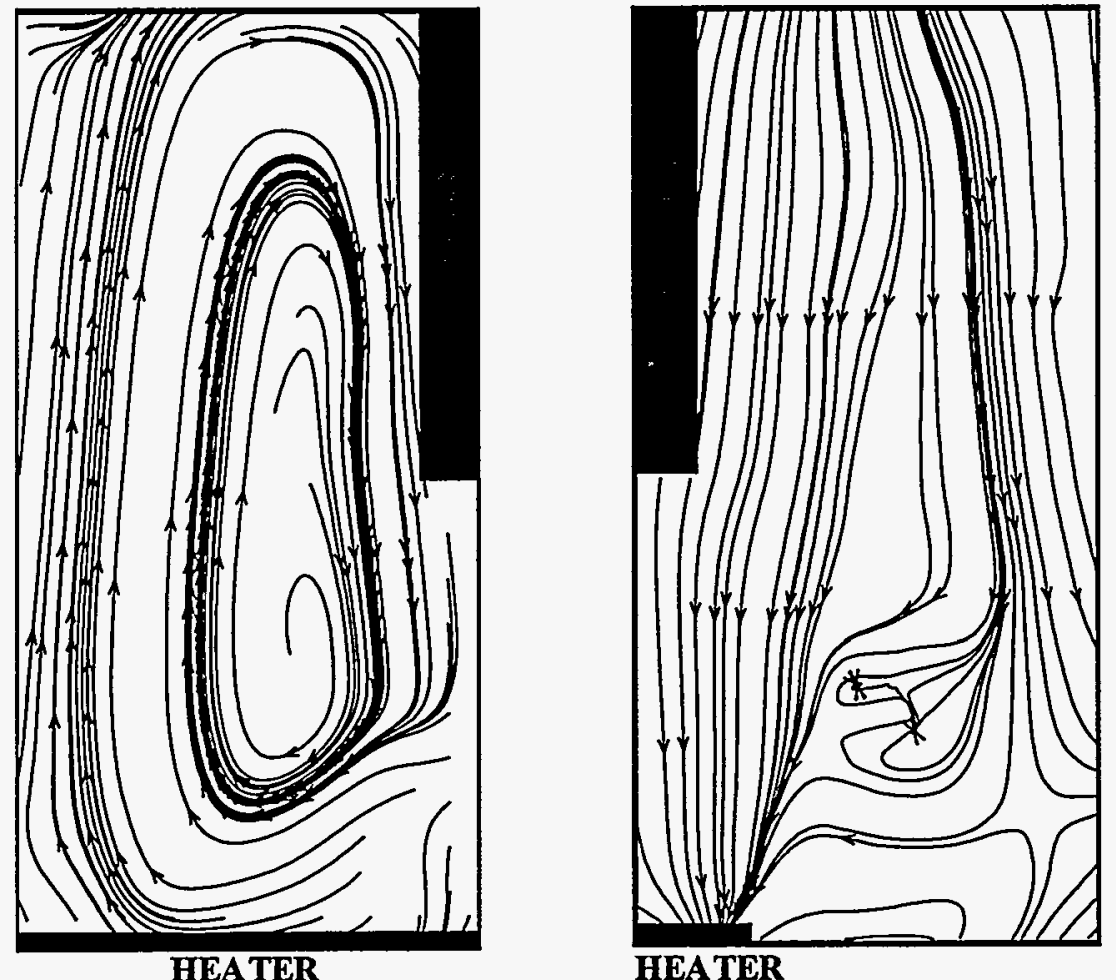

Figure 2.22. Streamlines calculated from PIV velocity fields given in Figure 2.20. Qualitative agreement with the computational simulation in Figure 2.21 can be seen. 
This page left intentionally blank 


\section{THREE-DIMENSIONAL PARTICLE IMAGE VELOCIMETRY}

\subsection{Introduction}

The measurement of 3D velocity vectors in 3D flows requires measurement of all three velocity components $\left(\mathrm{u}_{\mathrm{x}}, \mathrm{u}_{\mathrm{y}}\right.$, and $\mathrm{u}_{\mathrm{z}}$ in the $\mathrm{x}, \mathrm{y}$, and $\mathrm{z}$ directions, respectively). Traditional 3D velocity measurement techniques include LDV and hot wire anemometry. Both of these techniques require complicated experimental configurations and expensive data acquisition and processing electronics, and make measurements at only a specific point in the flow field. To measure the full flow field requires traversing the point measurement region, effectively limiting applicability to steady flows.

Standard two-dimensional PIV provides a detailed planar velocity map, well suited to 2D flow fields, but cannot measure the out-of-plane velocity component of a 3D flow. In fact, errors arise in 2D PIV when out-of-plane motion becomes appreciable. Obviously, particles entering or leaving the illuminated plane during the image data acquisition period will weaken the correlation and lead to the possibility of false vector identification.

A techniques for 3D PIV will be investigated here, based on volume illumination of the flow field, recording particle motion with multiple cameras.

\subsection{Three-Dimensional PIV}

The method of 3D PIV chosen here is stereo views taken of the flow using multiple cameras (see Figure 3.1). This technique has been applied by other researchers, but according to Grant (1994), the use of stereo views "presents problems in image matching that have a timeless appeal and provide potential for future work." In addition, Grant states that "accurate calibration of the relationship between object and images is still the subject of discussion." The results of this investigation support these statements.

\subsubsection{Camera Calibration and Particle Combination Theory}

Before tracking the particles through three dimensions, it is necessary to combine the separate camera image frames into a three-dimensional space frame. Since it is not obvious which particle is which in the separate image frames, this step can introduce error into the analysis of the data. Therefore, three cameras are normally used to minimize the error in locating the seeds. Linearized equations relate the location of a particle in a two-dimensional image axis to its three-dimensional world position (Gonzalez and Wintz, 1987). In order to use these equations, however, the precise location and orientation of the camera must be determined. These can be found by measuring the exact position and rotation of the camera and adjusting them for refraction, or they can be 


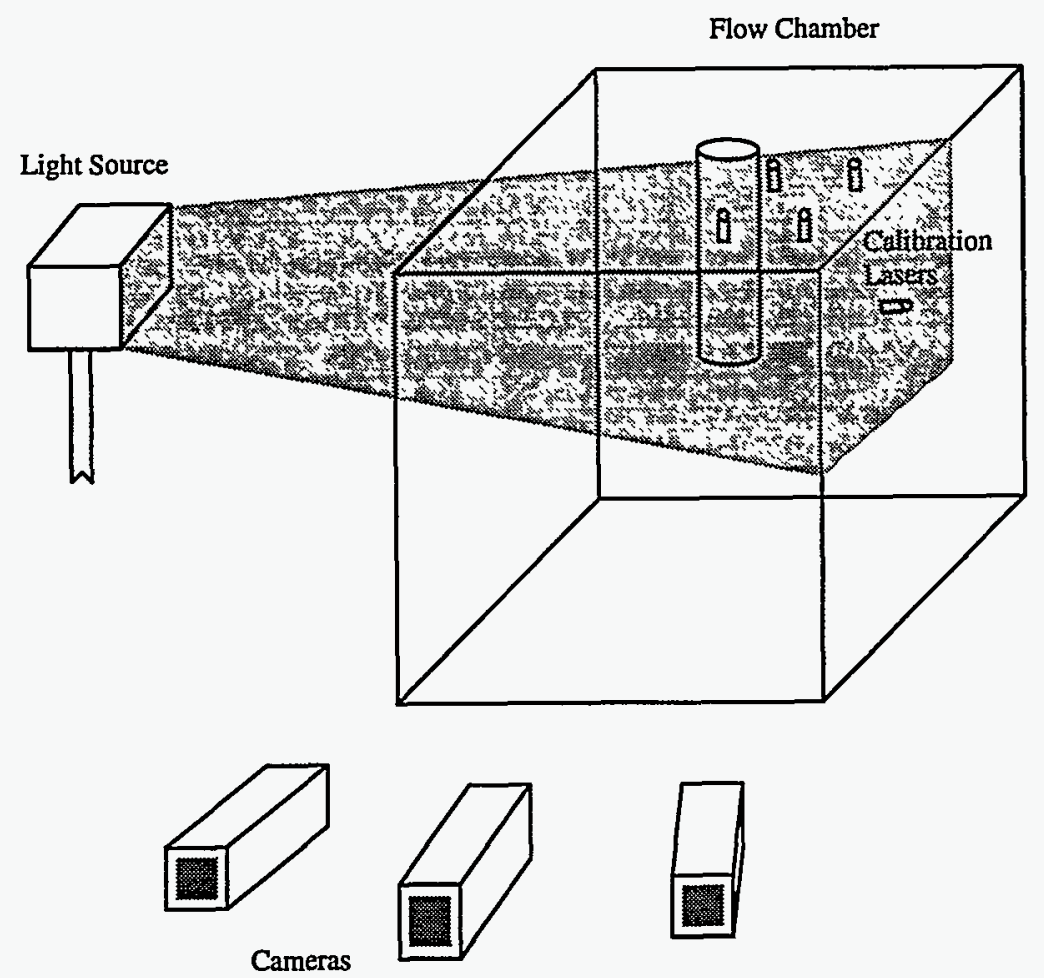

Figure 3.1 Three-Dimensional PIV set-up.

calculated by recording an image of known world positions. The method for calculating the variables from known world and camera positions is detailed here.

The equations relating a position in the world to a position in the camera are nonlinear. This is because of the perspective change through the focal point. However, by using a linear transformation, the process of relating the camera to the world is greatly simplified. Equation 1 displays the coordinates of a world point, $\mathrm{W}$. This point is transformed into a homogenous world 
point, $\mathrm{W}_{\mathrm{h}}$, (Eq. 2), by the introduction of a new dimension $\mathrm{k}_{\mathrm{w}}$. To return to a normal world point, the first three indices of the homogenous world vector are simply divided by the fourth index.

$$
\begin{gathered}
W=\left[\begin{array}{l}
\mathrm{X}_{\mathrm{w}} \\
Y_{\mathrm{w}} \\
Z_{\mathrm{w}}
\end{array}\right] \\
W_{\mathrm{h}}=\left[\begin{array}{c}
\mathrm{k}_{\mathrm{w}} \mathrm{X}_{\mathrm{w}} \\
\mathrm{k}_{\mathrm{w}} \mathrm{Y}_{\mathrm{w}} \\
\mathrm{k}_{\mathrm{w}} \mathrm{Z}_{\mathrm{w}} \\
\mathrm{k}_{\mathrm{w}}
\end{array}\right]
\end{gathered}
$$

By using $W_{h}$, the homogenous camera point, $C_{h}$, can be obtained simply by multiplying $W_{h}$ by the perspective transformation matrix, $P$, given by Eq. 3 .

$$
P=\left[\begin{array}{cccc}
1 & 0 & 0 & 0 \\
0 & 1 & 0 & 0 \\
0 & 0 & 1 & 0 \\
0 & 0 & -1 / \lambda & 1
\end{array}\right]
$$

The perspective change is caused by all the light passing through the focal point of the camera before intersecting the camera $\mathrm{XY}$ axes. This focal point is separated from the camera origin by the focal length, $\lambda$, which is perpendicular to camera $X Y$ axes and is measured in camera units. This only works if the world axes and camera axes are perfectly aligned. Since this is too restrictive, especially with multiple cameras, it is necessary to use other transformations to first scale, translate, and rotate the world axes to match the camera axes. This is done with the scaling transformation matrix, $\mathrm{S}$, the rotation transformation matrix, $\mathrm{R}$, and the translation transformation matrix, $T$, shown in Equations 4, 5, and 6. These are then combined with $P$ into the complete transformation matrix, A, as shown in Eq. 7. The scaling factors $S_{x}, S_{y}$, and $S_{z}$ multiply the world axes to obtain the camera axes, and are measured in camera units/world units. The angles $\alpha, \beta$, and 
measure the rotation around the $\mathrm{x}, \mathrm{y}$, and $\mathrm{z}$ axes respectively needed to match up the camera and world axes. The distances $\Delta x, \Delta y$, and $\Delta z$ measure the distance between the world and camera origins in world units.

$$
\begin{gathered}
S=\left[\begin{array}{llll}
S_{x} & 0 & 0 & 0 \\
0 & S_{y} & 0 & 0 \\
0 & 0 & S_{z} & 0 \\
0 & 0 & 0 & 1
\end{array}\right] \\
R=R_{\beta} R_{\alpha} R_{\theta}=\left[\begin{array}{cccc}
\cos \beta & 0 & -\sin \beta & 0 \\
0 & 1 & 0 & 0 \\
\sin \beta & 0 & \cos \beta & 0 \\
0 & 0 & 0 & 1
\end{array}\right]\left[\begin{array}{cccc}
1 & 0 & 0 & 0 \\
0 & \cos \alpha & \sin \alpha & 0 \\
0 & -\sin \alpha & \cos \alpha & 0 \\
0 & 0 & 0 & 1
\end{array}\right]\left[\begin{array}{cccc}
\cos \theta & \sin \theta & 0 & 0 \\
-\sin \theta & \cos \theta & 0 & 0 \\
0 & 0 & 1 & 0 \\
0 & 0 & 0 & 1
\end{array}\right] \\
T=\left[\begin{array}{cccc}
1 & 0 & 0 & \Delta x \\
0 & 1 & 0 & \Delta y \\
0 & 0 & 1 & \Delta z \\
0 & 0 & 0 & 1
\end{array}\right] \\
A=P S R T
\end{gathered}
$$

Using $A$, it is possible to transform any world point into a camera point for a camera located anywhere. $C_{h}$ is obtained simply by multiplying $W_{h}$ by $A$, as shown in Eq. 8 . The camera point, $C$, is then obtained by dividing the first three indices of $C_{h}$ by the fourth index, as shown in Eq. 9.

$$
\begin{gathered}
\mathrm{C}_{\mathrm{h}}=\left[\begin{array}{l}
\mathrm{k}_{\mathrm{c}} \mathrm{X}_{\mathrm{c}} \\
\mathrm{k}_{\mathrm{c}} \mathrm{Y}_{\mathrm{c}} \\
\mathrm{k}_{\mathrm{c}} \mathrm{Z}_{\mathrm{c}} \\
\mathrm{k}_{\mathrm{c}}
\end{array}\right]=A W_{\mathrm{h}}=\left[\begin{array}{l}
\mathrm{k}_{\mathrm{w}}\left(\mathrm{a}_{11} \mathrm{X}_{\mathrm{w}}+\mathrm{a}_{12} \mathrm{Y}_{\mathrm{w}}+\mathrm{a}_{13} \mathrm{Z}_{\mathrm{w}}+\mathrm{a}_{14}\right) \\
\mathrm{k}_{\mathrm{w}}\left(\mathrm{a}_{21} \mathrm{X}_{\mathrm{w}}+\mathrm{a}_{22} \mathrm{Y}_{\mathrm{w}}+\mathrm{a}_{23} \mathrm{Z}_{\mathrm{w}}+\mathrm{a}_{24}\right) \\
\mathrm{k}_{\mathrm{w}}\left(\mathrm{a}_{31} \mathrm{X}_{\mathrm{w}}+\mathrm{a}_{32} \mathrm{Y}_{\mathrm{w}}+\mathrm{a}_{33} \mathrm{Z}_{\mathrm{w}}+\mathrm{a}_{34}\right) \\
\mathrm{k}_{\mathrm{w}}\left(\mathrm{a}_{41} \mathrm{X}_{\mathrm{w}}+\mathrm{a}_{42} \mathrm{Y}_{\mathrm{w}}+\mathrm{a}_{43} \mathrm{Z}_{\mathrm{w}}+\mathrm{a}_{44}\right)
\end{array}\right] \\
\mathrm{C}=\left[\begin{array}{l}
\mathrm{X}_{\mathrm{c}} \\
\mathrm{Y}_{\mathrm{c}} \\
\mathrm{Z}_{\mathrm{c}}
\end{array}\right]=\left[\begin{array}{l}
\left(\mathrm{a}_{11} \mathrm{X}_{\mathrm{w}}+\mathrm{a}_{12} \mathrm{Y}_{\mathrm{w}}+\mathrm{a}_{13} \mathrm{Z}_{\mathrm{w}}+\mathrm{a}_{14}\right) /\left(\mathrm{a}_{41} \mathrm{X}_{\mathrm{w}}+\mathrm{a}_{42} \mathrm{Y}_{\mathrm{w}}+\mathrm{a}_{43} \mathrm{Z}_{\mathrm{w}}+\mathrm{a}_{44}\right) \\
\left(\mathrm{a}_{21} \mathrm{X}_{\mathrm{w}}+\mathrm{a}_{22} \mathrm{Y}_{\mathrm{w}}+\mathrm{a}_{23} \mathrm{Z}_{\mathrm{w}}+\mathrm{a}_{24}\right) /\left(\mathrm{a}_{41} \mathrm{X}_{\mathrm{w}}+\mathrm{a}_{42} \mathrm{Y}_{\mathrm{w}}+\mathrm{a}_{43} \mathrm{Z}_{\mathrm{w}}+\mathrm{a}_{44}\right) \\
\left(\mathrm{a}_{31} \mathrm{X}_{\mathrm{w}}+\mathrm{a}_{32} \mathrm{Y}_{\mathrm{w}}+\mathrm{a}_{33} \mathrm{Z}_{\mathrm{w}}+\mathrm{a}_{34}\right) /\left(\mathrm{a}_{41} \mathrm{X}_{\mathrm{w}}+\mathrm{a}_{42} \mathrm{Y}_{\mathrm{w}}+\mathrm{a}_{43} \mathrm{Z}_{\mathrm{w}}+\mathrm{a}_{44}\right)
\end{array}\right]
\end{gathered}
$$


Thus it is possible to transform a known world point into a camera point for a camera with known focal length, scaling, rotation, and location. This is useful for creating synthetic data, but not much else. However, we can rearrange the equations in order to locate the cameras, and then use that information to do the reverse transformation. By rearranging the equations for $X_{c}$ and $Y_{c}$, Equations 10 and 11 can be obtained.

$$
\begin{aligned}
& \left(a_{11} X_{w}+a_{12} Y_{w}+a_{13} Z_{w}+a_{14}\right)-X_{c}\left(a_{41} X_{w}+a_{42} Y_{w}+a_{43} Z_{w}+a_{44}\right)=0 \\
& \left(a_{21} X_{w}+a_{22} Y_{w}+a_{23} Z_{w}+a_{24}\right)-Y_{c}\left(a_{41} X_{w}+a_{42} Y_{w}+a_{43} Z_{w}+a_{44}\right)=0
\end{aligned}
$$

Using these two equations with known camera and world points, it is possible to solve for the coefficients of the transformation matrix, A. With six known world points and their corresponding camera points, the twelve coefficients used can be solved for. However, since the equations are homogeneous, it is necessary to fix one of the coefficients, with the other eleven being proportional to it. Once these coefficients are found, the various variables that make them up can be solved for using non-linear equations. These variables are the focal length, scaling, rotation, and location of the camera.

Once the variables that make up the coefficients of the transformation matrix are found, then all sixteen coefficients can be solved for, and the inverse process can be found. The inverse process is simply $\mathrm{A}^{-1}$, and it can be used for solving for the homogenous world points as shown in Equations 12 and 13.

$$
\begin{gathered}
A^{-1}=T^{-1} R^{-1} S^{-1} P^{-1} \\
W_{h}=A^{-1} C_{h}
\end{gathered}
$$

However, when the inverse process is solved it requires $\mathrm{Z}_{c}$, which cannot be measured using one camera. So, instead of using the direct inverse process, a slightly different process is used. The location of a homogenous camera point is found on the world axes using Eq. 14. Similarly, the location of the focal point can be found in world coordinates as shown in Eq. 15. Neither of these equations uses the perspective transformation, since the actual position of these points is desired. 
The non-homogeneous position can be solved for simply by dividing by the fourth index, as before. Thus the actual position of the camera point and the focal point is obtained on the world axes.

$$
\begin{aligned}
& W_{c h}=\left[\begin{array}{c}
k_{w} X_{w c} \\
k_{w} Y_{w c} \\
k_{w} Z_{w c} \\
k_{w}
\end{array}\right]=T^{-1} R^{-1} S^{-1}\left[\begin{array}{c}
k_{c} X_{c} \\
k_{c} Y_{c} \\
0 \\
k_{c}
\end{array}\right] \\
& W_{f h}=\left[\begin{array}{c}
k_{w} X_{w f} \\
k_{w} Y_{w f} \\
k_{w} Z_{w f} \\
k_{w}
\end{array}\right]=T^{-1} R^{-1} S^{-1}\left[\begin{array}{c}
0 \\
0 \\
k_{c} \lambda \\
k_{c}
\end{array}\right]
\end{aligned}
$$

Using these points, a line can be drawn on the world axes, as shown in Eq. (16). The world point that corresponds to the camera point used to make this line must be somewhere along this line. A similar line can be drawn from a corresponding point in a second camera. Where these two lines intersect the original world point is located. This is demonstrated in two dimensions by Fig. 3.2.

$$
\mathrm{L}_{\mathrm{h}}=\left[\begin{array}{l}
\mathrm{X}_{\mathrm{L}} \\
\mathrm{Y}_{\mathrm{L}} \\
\mathrm{Z}_{\mathrm{L}}
\end{array}\right]=\left[\begin{array}{l}
\mathrm{X}_{\mathrm{wf}} \\
\mathrm{Y}_{\mathrm{wf}} \\
\mathrm{Z}_{\mathrm{wf}}
\end{array}\right]+\mathrm{j}\left[\begin{array}{l}
\mathrm{X}_{\mathrm{wc}} \\
\mathrm{Y}_{\mathrm{wc}} \\
\mathrm{Z}_{\mathrm{wc}}
\end{array}\right]
$$

The process is more complicated in three dimensions. In three dimensions, the lines should still theoretically cross, but with the introduction of error they no longer cross. So instead of finding the intersection of the two lines, differentiation is used to find where the lines are closest to each other. The world point is then located centered between the two lines. Another problem arises from the fact that the correspondence of points in one camera to points in the other camera is unknown. Thus, every point in the second camera must be used to draw a line to find the best match for each point in the first camera. The point in the second camera whose line comes closest to the line created by the point in the first camera is assumed to be the corresponding point. With only two cameras, it is impossible to discriminate wrong matches from correct matches, so a third camera is used to check. If all three cameras agree on the point, then it is used. If not, then the point is dropped. By this method, any point produced by the process should be approximately in the correct position. 


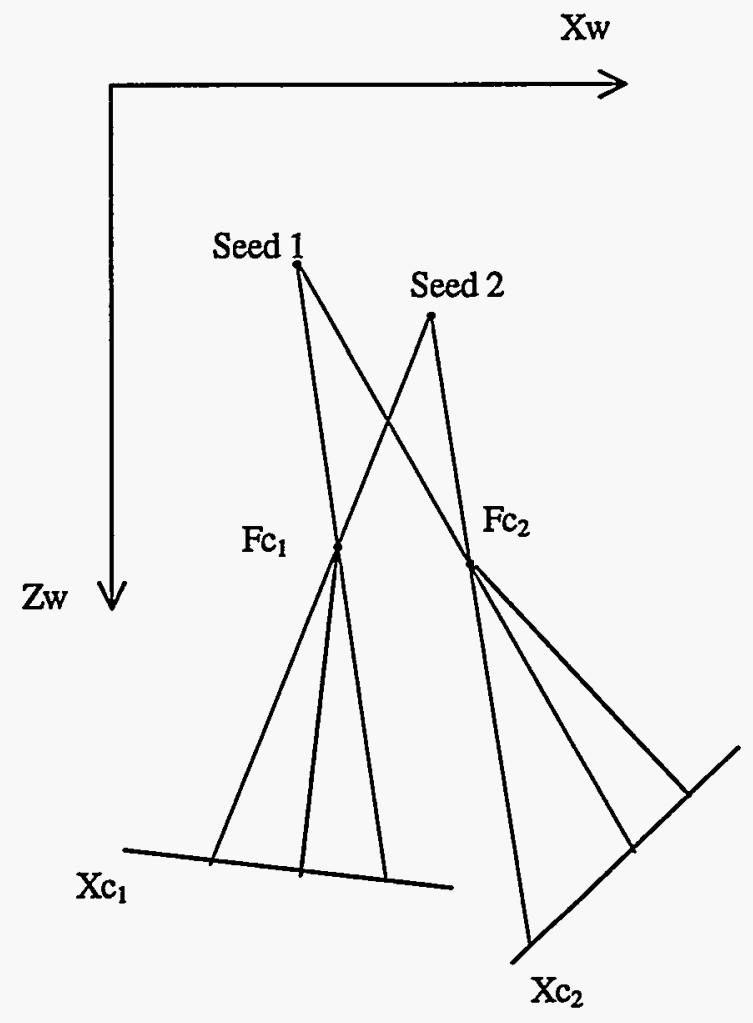

Figure 3.2 Simple model of particle determination.

\subsubsection{Theory for Tracking Programs}

Just as in two-dimensional PIV (see Ch. 2), two methods have been developed to obtain the displacement of multiple particles between flow images. They are the cross-correlation tracking (CCT) algorithm and the multi-frame particle tracking (MFT) algorithm. These two methods are used in the PST3D and TP3D programs, respectively (see Appendices for details).

The 3D cross-correlation tracking (CCT) algorithm is identical to the 2D CCT algorithm except that comparison volumes, rather than areas, are used. The particle velocity is found by determining the correspondence between particles in the comparison volumes of the two frames. Every particle belongs to a characteristic group that has a specific 3D local distribution pattern in the first space frame and multiple candidate patterns in the second frame. Each second frame candidate's comparison volume is shifted over the first frame comparison volume such that the original particle's center is positioned over the candidate particle's center. The particle in the first frame corresponds to the particle in the second image that maintains the most similar pattern within the comparison volume, providing that the local pattern of the distributed particles changes little within the time that the original images were taken. This method is particularly useful when only two sequential frames are available, and the MFT method cannot be performed. 
The CCT algorithm correlates between two frames of data. A search volume is formed in the second frame, centered on the location of the original particle in the first frame. The size of this volume is determined by estimating the maximum possible movement for particles between frames. Each Frame 2 particle in this volume is a candidate for being the original particle as moved by the flow. A comparison volume is then created around each candidate, encompassing at least ten other particles in Frame 2. A comparison volume of the same size is created in Frame 1, centered on the original Frame 1 particle. The two volumes are then compared by shifting the center of the candidate particle over the center of the original particle. This comparison is represented by the correlation coefficient, $\mathrm{C}_{\mathrm{ij}}$, which measures the overlap of the particles in the two volumes. $A C_{i j}$ value of 1 indicates a perfect correspondence between two volumes; the closer the value is to 1 , the more likely that it is the correct particle match. $\mathrm{AC}_{\mathrm{ij}}$ value of 1 is only possible if each spot is the same size in both comparison volumes, and each spot is centered in the same point when shifted to overlap the two volumes. The correlation coefficient is calculated just as in the $2 \mathrm{D}$ algorithm.

The 3D MFT algorithm is identical to the $2 \mathrm{D}$ version, except that search volumes are used in place of search areas. The sequential space frames obtained by particle determination contain the information necessary for the three-dimensional tracking scheme. The MFT routine tracks particles through four frames directly, and then it combines the four frame tracks to indirectly track through all the frames available. Several possible tracks exist for each particle in the first interrogation volume. The search volume in the second frame for a particle starting in the first frame is a sphere of radius, $\mathrm{R} 1$, determined by a rough estimate of the maximum velocity of the flow field. The search volumes in the third and fourth frames have their radius, R2, determined by a rough estimate of the maximum acceleration of the flow field. The center of each search volume in frame 3 is found by projecting out along the straight line, $\mathrm{L}_{1-2}$, of a possible track for each particle found in the search volume of frame 2. For each particle found in the search volume of frame 3 , the center of a search volume in frame 4 is determined by using the length of the track from frame 2 to $3, L_{2-3}$, and the angles created by the track from frame 1 to $3, \theta_{1-3}$ and $\Phi_{1-3}$. This results in several different possible tracks for each particle in frame one, the best of which is used as the actual track for that particle, as determined by the lowest sigma value. If different tracks originating in the same frame share a particle, then the one with the highest sigma value is disposed of as incorrect.

\subsection{D Validation}

\subsubsection{Synthetic Data}

To verify the ability of these two tracking algorithms to track particle movement in three dimensions, two types of tests were conducted. The first test was with synthetic data, and the second with experimental data. To produce the three-dimensional synthetic data, a standard flow was required to create sets of particles at known positions and move them from frame to frame. Simulated inviscid flow around a sphere and simulated flow to a drain were used for this purpose. 
Exact analytical solutions were used to move the simulated tracer particles. The tracking method was then performed using these frames of data to produce particle tracks. Comparisons were made between the tracks produced by the algorithms and the known particle tracks.

By comparing the tracks created by the tracking algorithms to the original synthetic tracks, the tracking algorithms can be evaluated (Henderson, 1994). Each algorithm was evaluated with two factors, yield and reliability. The yield is the number of tracks found divided by the number of tracks produced, while the reliability is the number of correct tracks divided by the number of tracks found. By multiplying the two factors together, the number of correct tracks per generated track is obtained. For the synthetic flow around a sphere, the CCT code has a higher yield but a lower reliability at high velocities than the MFT code. Based on synthetic drain flow, the CCT generally has a higher yield but at the cost of lower reliability. Analysis was also performed based on the number of tracks, but it did not show a change in the performance of the codes until more than $\mathbf{5 0 0 0}$ tracks per frame were analyzed.

The second validation was performed by examining particle motion on the surface of a spinning disk. For both two-dimensional (flat) and three-dimensional (tilted) motions, the PIV algorithms were shown to work well.

\subsubsection{Three-Dimensional Convection Experiment Set-Up}

The description of the magma simulant flow chamber experiment was included in the previous chapter for the two-dimensional PIV set-up. No changes were made to the chamber except to lower the constant-temperature cooling plate and cylinder to $10^{\circ} \mathrm{C}$ and the heater plate to $30^{\circ} \mathrm{C}$.

Figure 3.1 shows the basic three-dimensional PIV set-up. Whereas a laser light source was used to illuminate the particles in a plane for 2D (planar) PIV, an overhead projector was used as a light source to provide an easily controllable volume of light for 3D PIV. Calibration points within the chamber were measured by capturing images of crossing calibration laser beams. These calibration lasers were $10 \mathrm{~mW}$ diode lasers ( $670 \mathrm{~nm}$ wavelength), whose beams could clearly be seen in the corn syrup. Pictures of the illuminated particles were captured using an array of three CCD cameras (Pulnix TM745). These pictures were directly captured by three EPIX frame grabber boards (4MEG video Model 10) and were saved as binary files for analysis on a 486/66 PC.

\subsubsection{Results and Discussion of Three-Dimensional Experiments}

The data obtained in the 3D experiment consisted of very sparse, unreliable vector fields. This was due to a number of experimental difficulties, including unanticipated strong refractive index gradients, which made absolute calibration impossible. In addition, diurnally induced temperature drifts and material degradation (carmelization) after prolonged heating contributed to the difficulties in acquiring accurate data. 
Thermal refraction was the biggest cause of problems. It caused errors in calibrating the cameras which then carried over to spot combination. With these calibration errors, the cross-correlation tracking routine could not function properly, as shown by an average $C_{i j}$ value of approximately 0.1 for the 3D experiments. By comparison, the 2D thermal convection experiments had average $C_{i j}$ values $>0.3$, and the $2 \mathrm{D}$ channel flow average $C_{i j}$ values were even higher.

\subsection{D PIV Validation Computations}

The computational fluid dynamics code FIDAP (Fluid Dynamics International, 1993) was used to simulate the three-dimensional natural-convection experiment discussed above. Figure 3.5 shows a schematic diagram of the experiment. The geometry under examination is a cubical box ( $2 \mathrm{~L}=56 \mathrm{~cm}$ side), within which a closed cylinder ( $7.6 \mathrm{~cm}$ diameter, $28 \mathrm{~cm}$ height) has been endmounted to the center of the top side. The top side of the box and cylinder are water-cooled, and a flush-mounted heater strip ( $56 \mathrm{~cm}$ length, $14 \mathrm{~cm}$ width) is centered on the bottom side of the box. All the other boundaries are insulated. The interior of this apparatus is completely filled with $42 /$ 43 corn syrup, which has been used by other investigators (e.g., Chu and Hickox, 1990) as a magma simulant because of its highly temperature-dependent viscosity. The conditions examined are cold and hot temperatures of $T_{C}=8.8^{\circ} \mathrm{C}$ and $T_{H}=31.7^{\circ} \mathrm{C}$, which correspond to a cold-to-hot viscosity ratio of 37, and cold Prandtl and Rayleigh numbers (based on the box half width) of $\mathrm{Pr}=6.7 \times 10^{6}$ and $R a=2.2 \times 10^{4}$, respectively, where $R a=\rho^{2} \operatorname{cg} \beta\left(T_{H}-T_{C}\right) L^{3} / \mu k$. The temperaturedependent material properties of $42 / 43$ corn syrup are given below, with the viscosity $\mu$ in Poise, the thermal conductivity $k$ in W/m.K, the specific heat $c$ in J/g.K, the density $\rho$ in $\mathrm{g} / \mathrm{cm}^{3}$, and the coefficient of thermal expansion $\beta$ in $K^{-1}$, where the temperature $T$ is in ${ }^{\circ} \mathrm{C}$.

$$
\begin{gathered}
\mu=a_{0} \exp \left[a_{1} \exp \left(-T / a_{2}\right)\right], a_{0}=0.2412, a_{1}=12.5867, a_{2}=55.7805 \\
k=b_{0}+b_{1} T, b_{0}=0.3724, b_{1}=3.034 \times 10^{-4} \\
c=c_{0}+c_{1} T+c_{2} T^{2}, c_{0}=2.2005, c_{1}=3.9532 \times 10^{-3}, c_{2}=-6.7883 \times 10^{-6} \\
\rho=\rho_{0}\left[1-\beta\left(T-T_{0}\right)\right], \rho_{0}=1.4255, \beta=4.1218 \times 10^{-4}, T_{0}=10
\end{gathered}
$$

Prior to simulation using FIDAP, this problem is formulated in nondimensional form. The following scalings are employed:

Table 2.2.1 Nondimensionalization of properties

\begin{tabular}{|l|l|l|l|l|}
\hline Quantity & Symbol & At $T_{C}=8.8^{\circ} \mathrm{C}$ & At $T_{H}=31.7^{\circ} \mathrm{C}$ & Scaled Value \\
\hline \hline Box Half Width & $L$ & $0.28 \mathrm{~m}$ & $0.28 \mathrm{~m}$ & 1 \\
\hline Density & $\rho$ & $1430.7 \mathrm{~kg} / \mathrm{m}^{3}$ & $1444.2 \mathrm{~kg} / \mathrm{m}^{3}$ & $1 / P r$ (cold, hot) \\
\hline
\end{tabular}


Table 2.2.1 Nondimensionalization of properties

\begin{tabular}{|l|l|l|l|l|}
\hline Quantity & Symbol & At $T_{C}=8.8^{\circ} \mathrm{C}$ & At $T_{H}=31.7^{\circ} \mathrm{C}$ & Scaled Value \\
\hline \hline Viscosity & $\mu$ & $1124.4 \mathrm{~kg} / \mathrm{m} \cdot \mathrm{s}$ & $30.1 \mathrm{~kg} / \mathrm{m} \cdot \mathrm{s}$ & $1 / R a^{1 / 2}$ (cold) \\
\hline Thermal Expansion Cf. & $\beta$ & $4.1238 \times 10^{-4} / \mathrm{K}$ & $4.1238 \times 10^{-4} / \mathrm{K}$ & $\operatorname{Pr}$ (cold, hot) \\
\hline Specific Heat & $c$ & $2234.8 \mathrm{~J} / \mathrm{kg} \cdot \mathrm{K}$ & $2319.0 \mathrm{~J} / \mathrm{kg} \cdot \mathrm{K}$ & $\operatorname{Pr}$ (cold) \\
\hline Thermal Conductivity & $k$ & $0.3751 \mathrm{~W} / \mathrm{m} \cdot \mathrm{K}$ & $0.3820 \mathrm{~W} / \mathrm{m} \cdot \mathrm{K}$ & $1 / R a^{1 / 2}$ (cold) \\
\hline Gravity & $g$ & $9.81 \mathrm{~m} / \mathrm{s}^{2}$ & $9.81 \mathrm{~m} / \mathrm{s}^{2}$ & 1 \\
\hline
\end{tabular}

Table 2.2.2 Nondimensionalization of variables

\begin{tabular}{|l|l|l|l|}
\hline Quantity & Symbol & Scaled Form & Numerical Value \\
\hline \hline Position & $\mathbf{x}$ & $\mathrm{x} / L$ & $L=0.28 \mathrm{~m}$ \\
\hline Velocity & $\mathbf{u}$ & $\mathbf{u} / U, U=R a^{1 / 2}(k / \rho c L)$ & $U=6.21 \times 10^{-5} \mathrm{~m} / \mathrm{s}$ \\
\hline Pressure & $p$ & $p / P, P=\rho g \beta\left(T_{H}-T_{C}\right) L$ & $P=37.1 \mathrm{~Pa}$ \\
\hline Temperature & $T$ & $\left(T-T_{C}\right) /\left(T_{H}-T_{C}\right)$ & $T_{C}=8.8^{\circ} \mathrm{C}, T_{H}=31.7^{\circ} \mathrm{C}$ \\
\hline
\end{tabular}

Table 2.2.3 Dimensionless parameters

\begin{tabular}{|l|l|l|}
\hline Parameter & Symbol & Value \\
\hline \hline Prandtl Number & $P r=\mu c / k$ & $6.7 \times 10^{6}$ (cold) \\
\hline Rayleigh Number & $R a=\rho^{2} c g \beta\left(T_{H}-T_{C}\right) L^{3} / \mu k$ & $2.2 \times 10^{4}$ (cold) \\
\hline Viscosity Ratio & $\mu_{C} / \mu_{H}$ & 37 \\
\hline Reynolds Number & $R e=\rho U L / \mu=R a^{1 / 2} / P r$ & $2.2 \times 10^{-5}$ (cold) \\
\hline Peclet Number & $P e=\rho c U L / k=R a^{1 / 2}$ & 148 (cold) \\
\hline
\end{tabular}

Several observations can be made based on the above scaling. First, the Reynolds number is very low. Thus, inertial effects such as "jetting" are nonexistent: the fluid motion is determined by a force balance between hydrostatic pressure, buoyancy, and viscous effects. Second, the Peclet number is rather high at cold conditions and is higher still at hot conditions, so the thermal boundary layers are thin. Since the flow is driven solely by buoyancy, inaccurate determination of the boundary layers would produce inaccurate heat transfer results, inaccurate buoyant forces, and inaccurate velocity values, even in the interior of the flow, so good resolution of the boundary layers is essential to obtaining an accurate flow solution. This combination of thin boundary layers and three-dimensionality makes this a computationally intense problem. 
The computational fluid dynamics code FIDAP is used to determine the velocity and temperature fields for the natural-convection flow problem outlined above and shown in Figure 3.5. Due to the symmetries in the problem, the computational domain consists of one-fourth of the physical domain. The flow is assumed to be steady, and the penalty method with a $10^{-5}$ penalty parameter is used to enforce incompressibility. The solution is computed on two different meshes (see Figure 3.6), where the more refined mesh has 1.5 times the nodal density of the more coarse mesh in all three coordinate directions. Comparisons of the results on these meshes indicates that a reasonable degree of grid independence is achieved (see Figure 3.7).

Figures 3.8-3.14 show contour plots of the field variables, and Figure 3.15 shows selected streamlines. Velocity components and temperature are seen to have thin boundary layers, as expected. The maximum speed is approximately 2.9 (corresponding to $0.2 \mathrm{~mm} / \mathrm{s}$ ), which indicates that the scaling used is physically sensible. The dominant contribution to the pressure is hydrostatic. The temperature is relatively uniform throughout the interior of the fluid (outside of the plume and the boundary layers), as would be expected for this high Peclet number. The streamlines in Figure 3.15 show a marked resemblance to flow-visualization pictures, shown in Figure 3.16. However, the fluid beneath the cylinder is moving downward toward the heater strip, contrary to initial expectations based on the two-dimensional simulations (without a cylinder) of Chu and Hickox (1990) and Hickox and Chu (1991) (see Torczynski et al. (1995)) After approaching the middle of the heater strip, the fluid subsequently moves out toward the sides of the box along the heater strip and up along the wall at the wall-heater junction (see also Figures 2.19 through 2.22 for $2 \mathrm{D}$ results). 

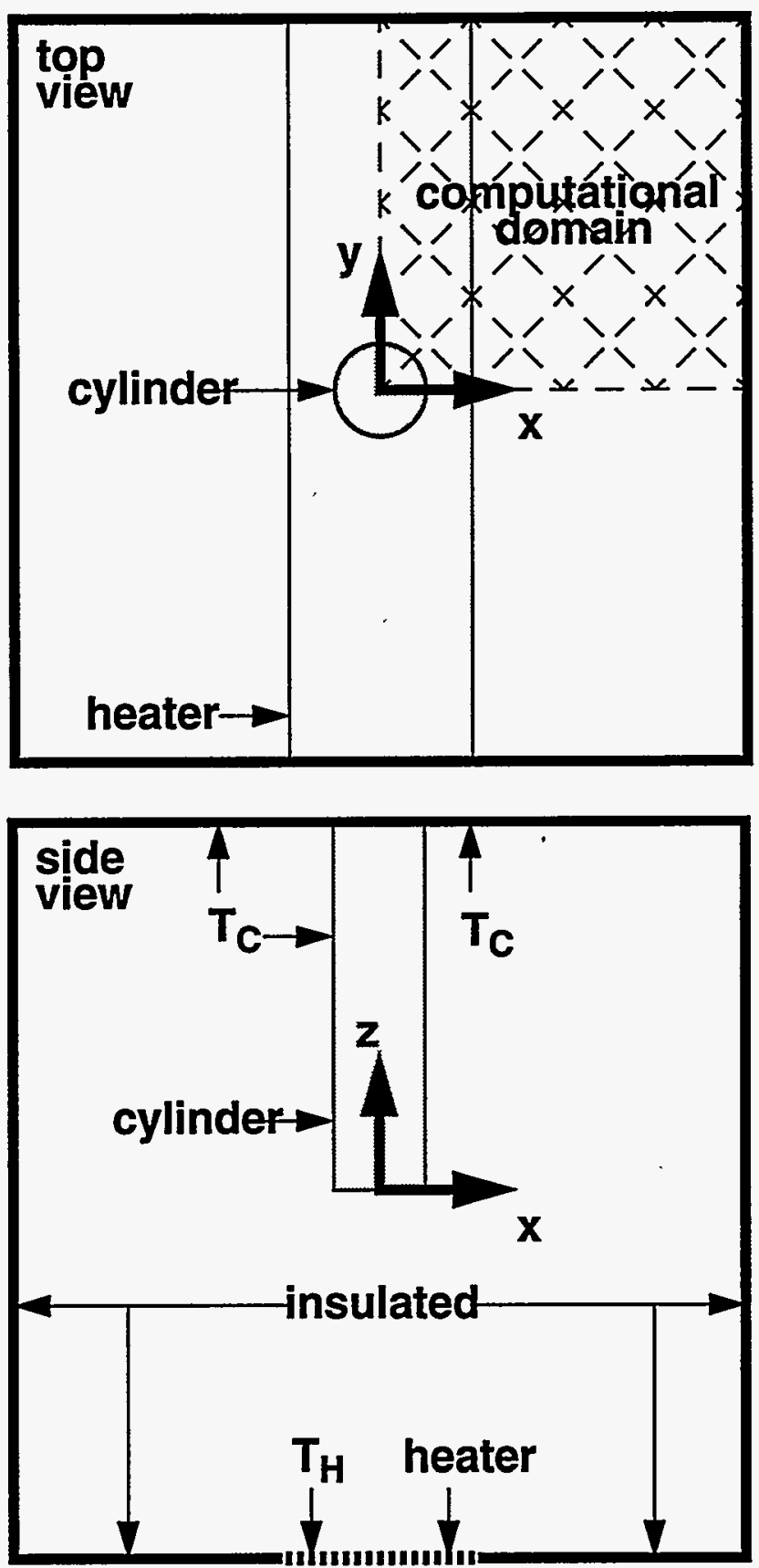

Figure 3.3 Schematic diagrams showing different views of the geometry. 

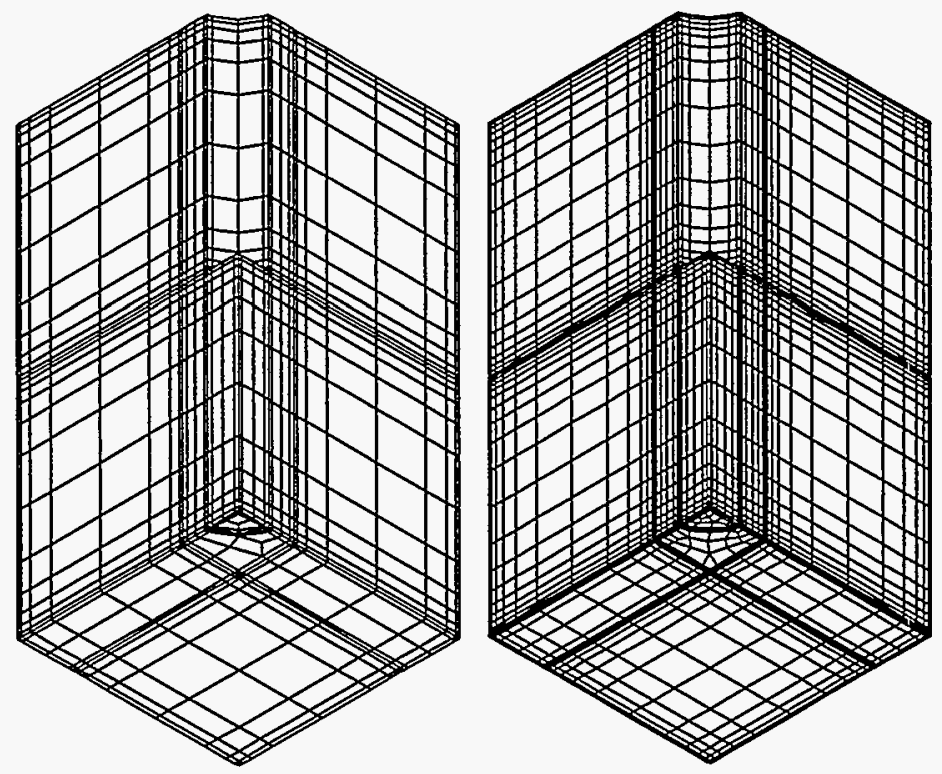

Figure 3.4 Left, coarse mesh. Right, refined mesh. Tri-linear brick elements.

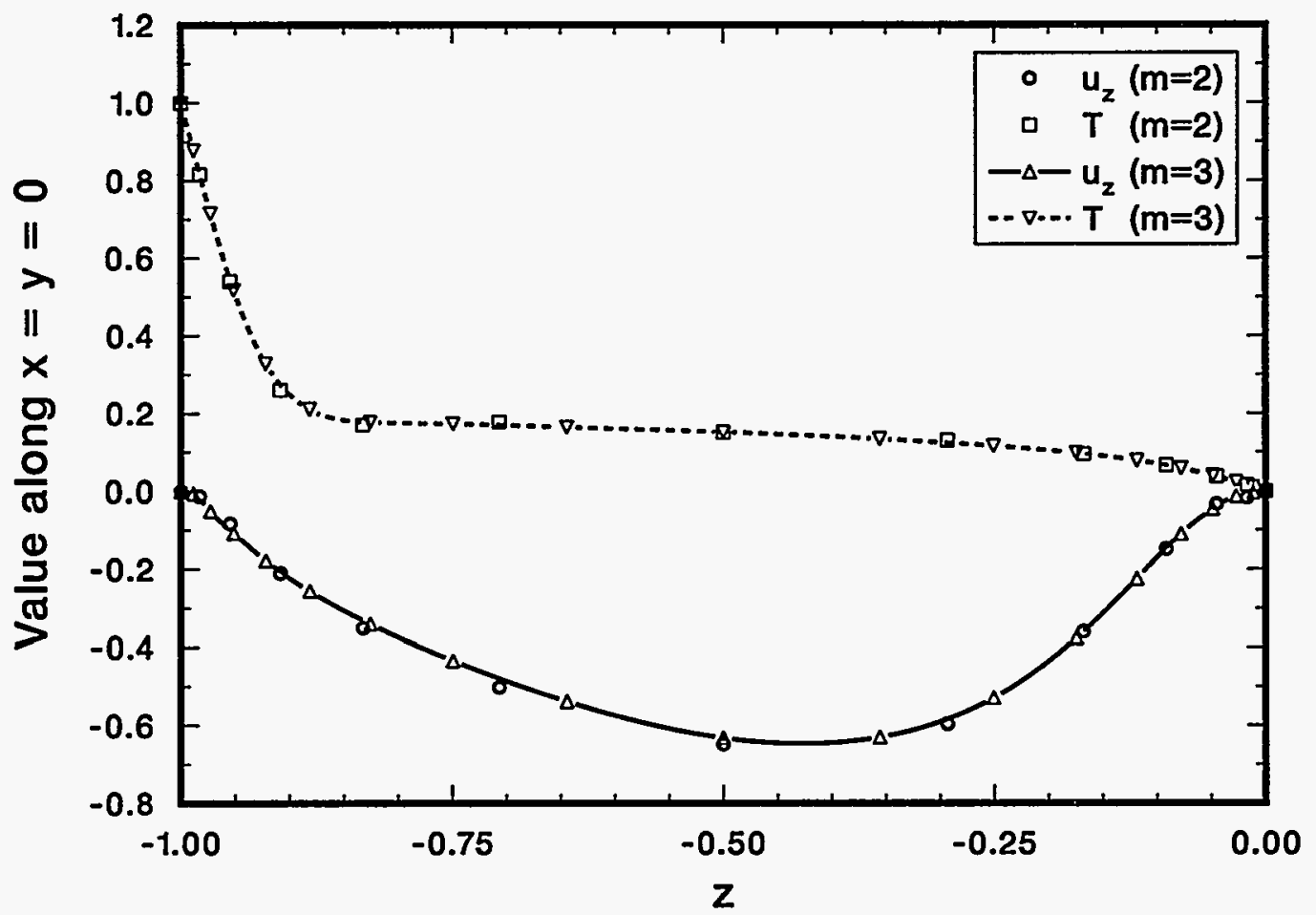

Figure 3.5 Effect of mesh on $\mathrm{u}_{\mathrm{z}}$ and $\mathrm{T}$ along $x=y=0$ (below the cylinder on its axis). 


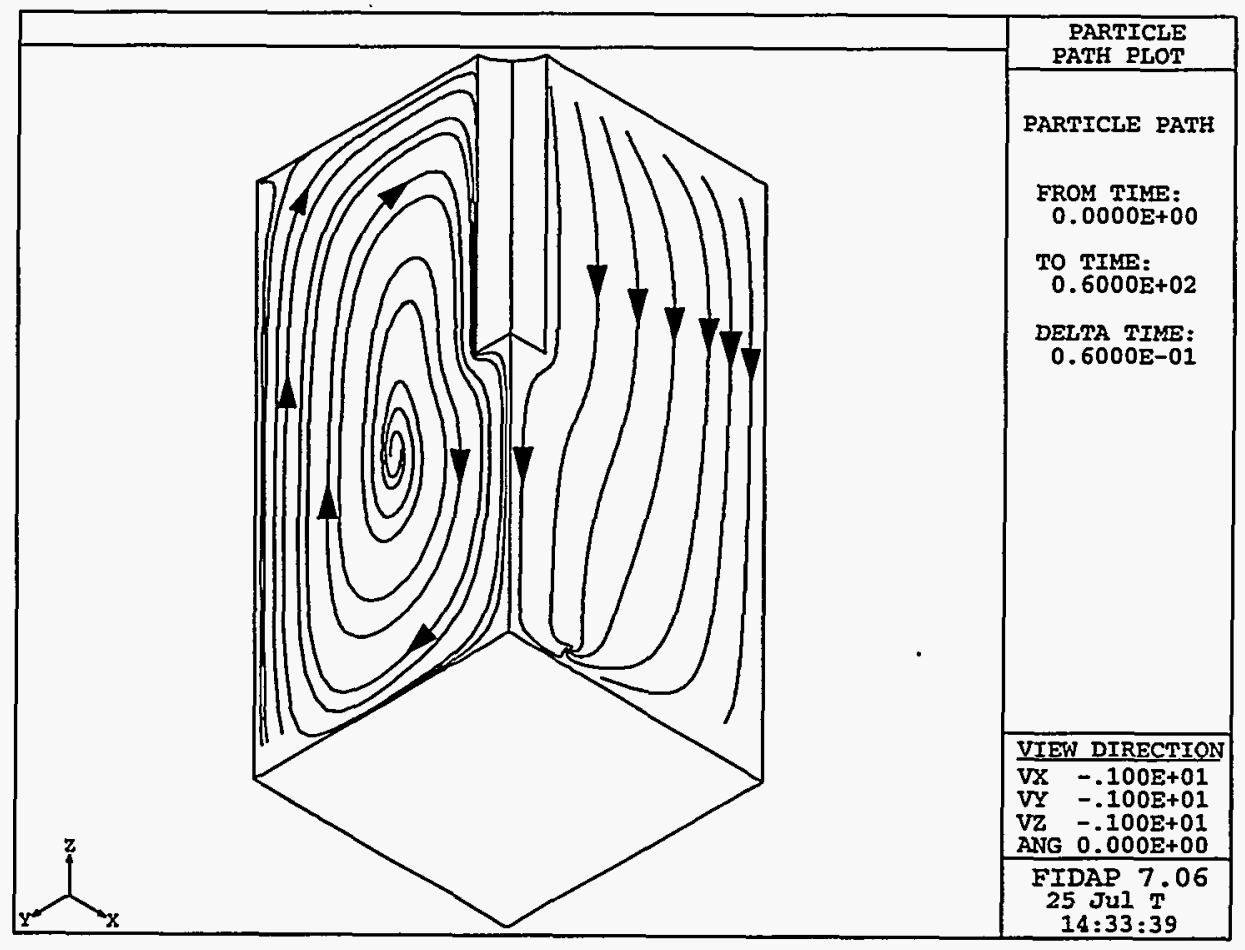

Figure 3.6 Selected streamlines on symmetry planes.

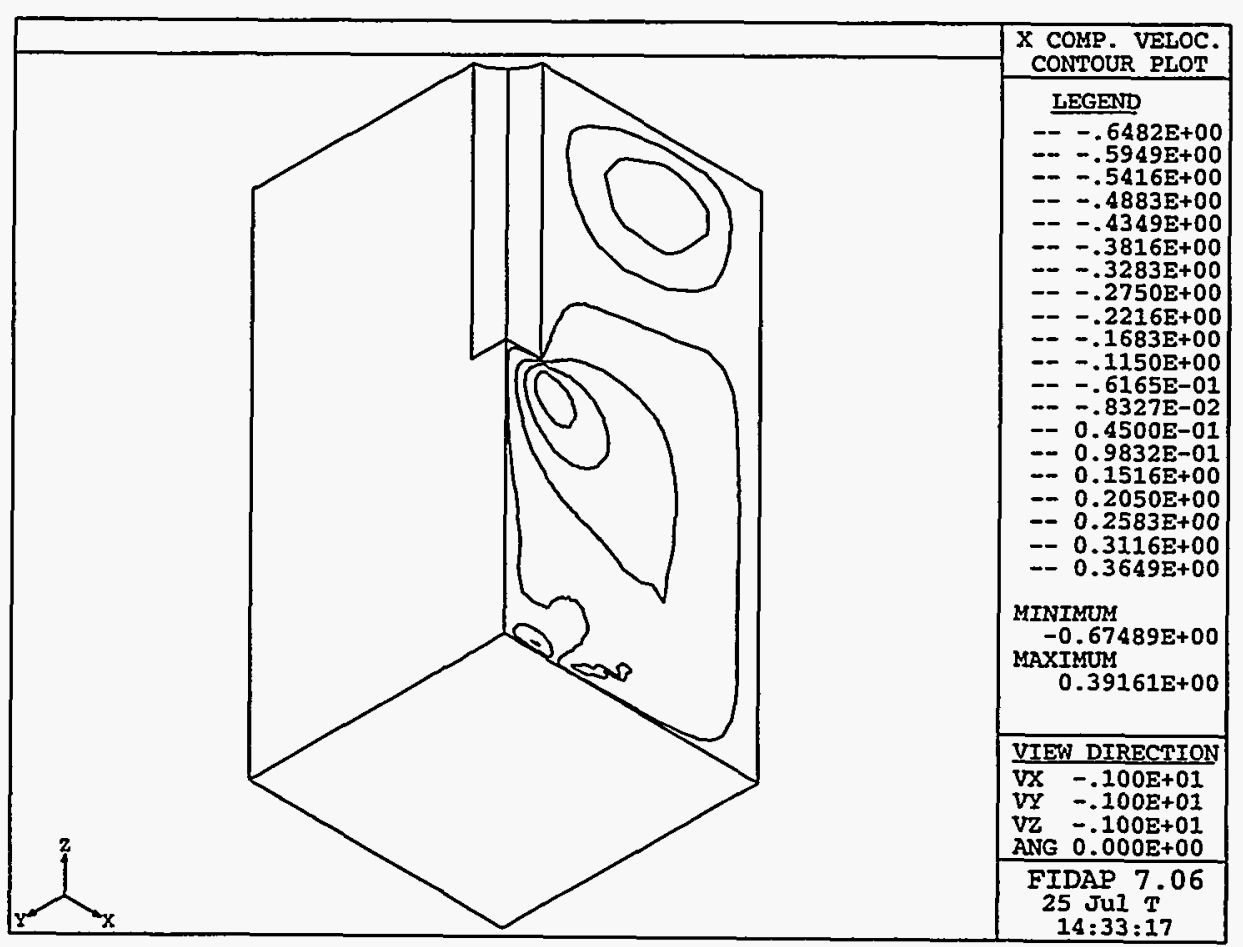

Figure 3.7 Contour plot of $\mathrm{u}_{\mathrm{x}}$. 


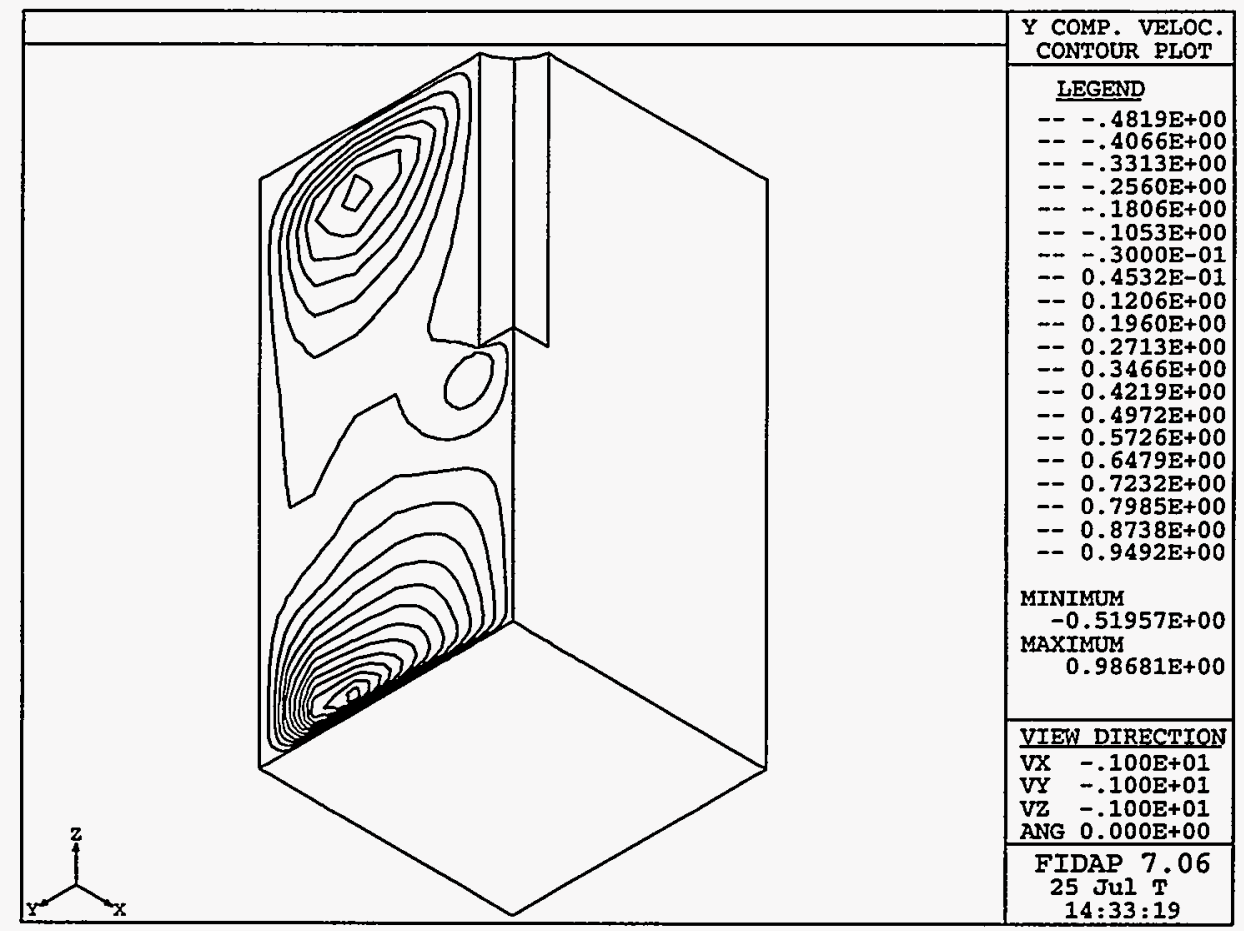

Figure 3.8 Contour plot of $u_{y}$.

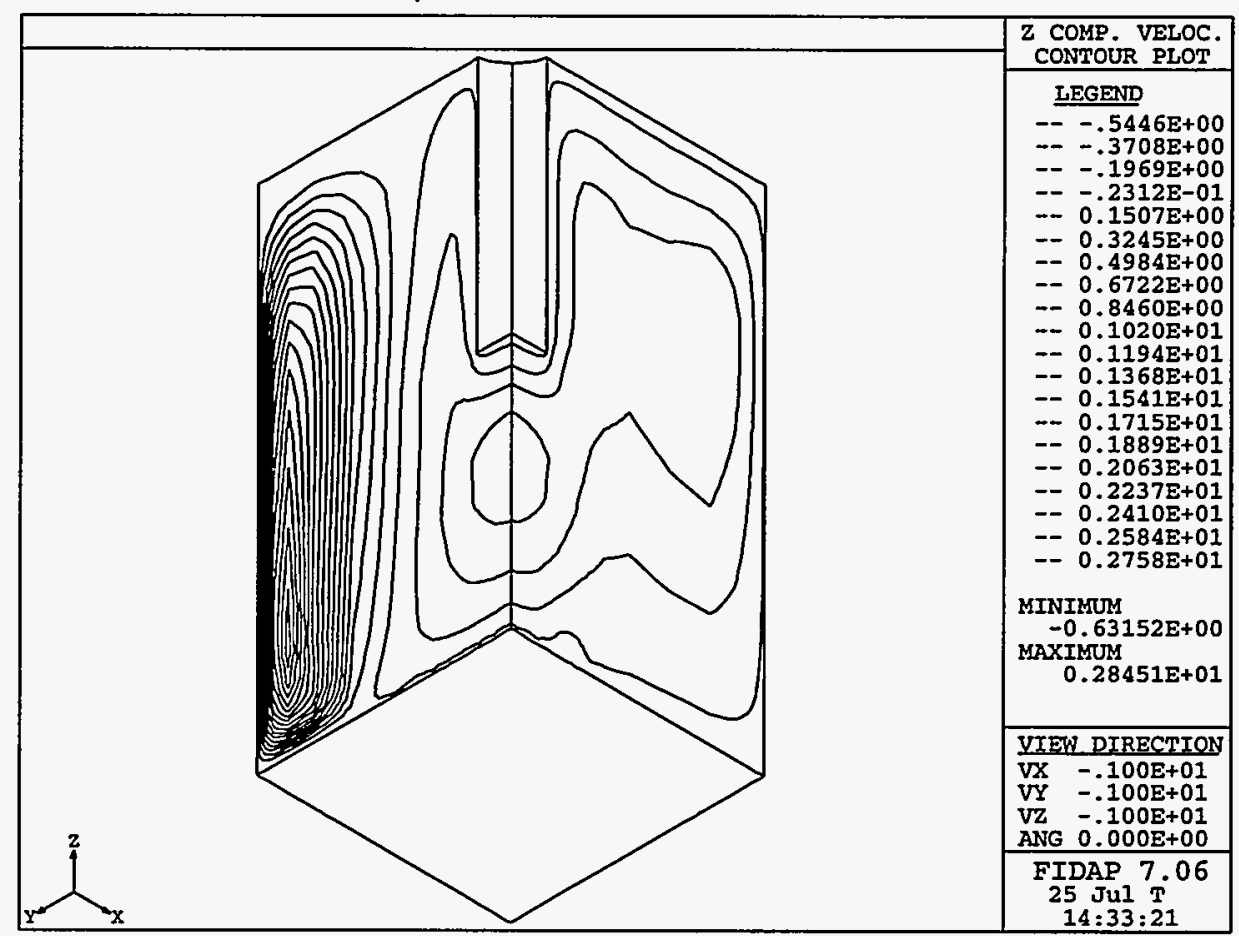

Figure 3.9 Contour plot of $\mathrm{u}_{\mathrm{z}}$. 


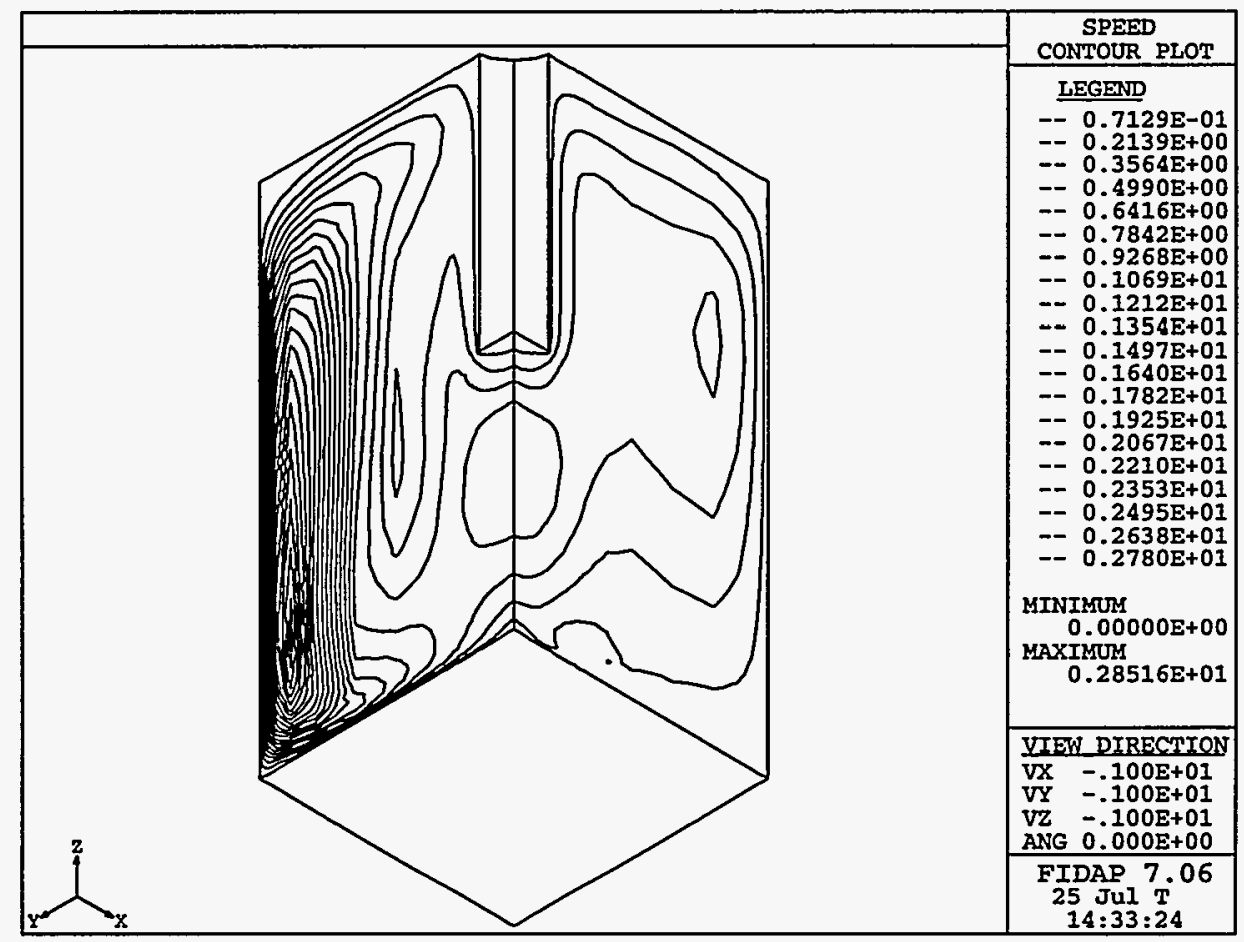

Figure 3.10 Contour plot of speed v.

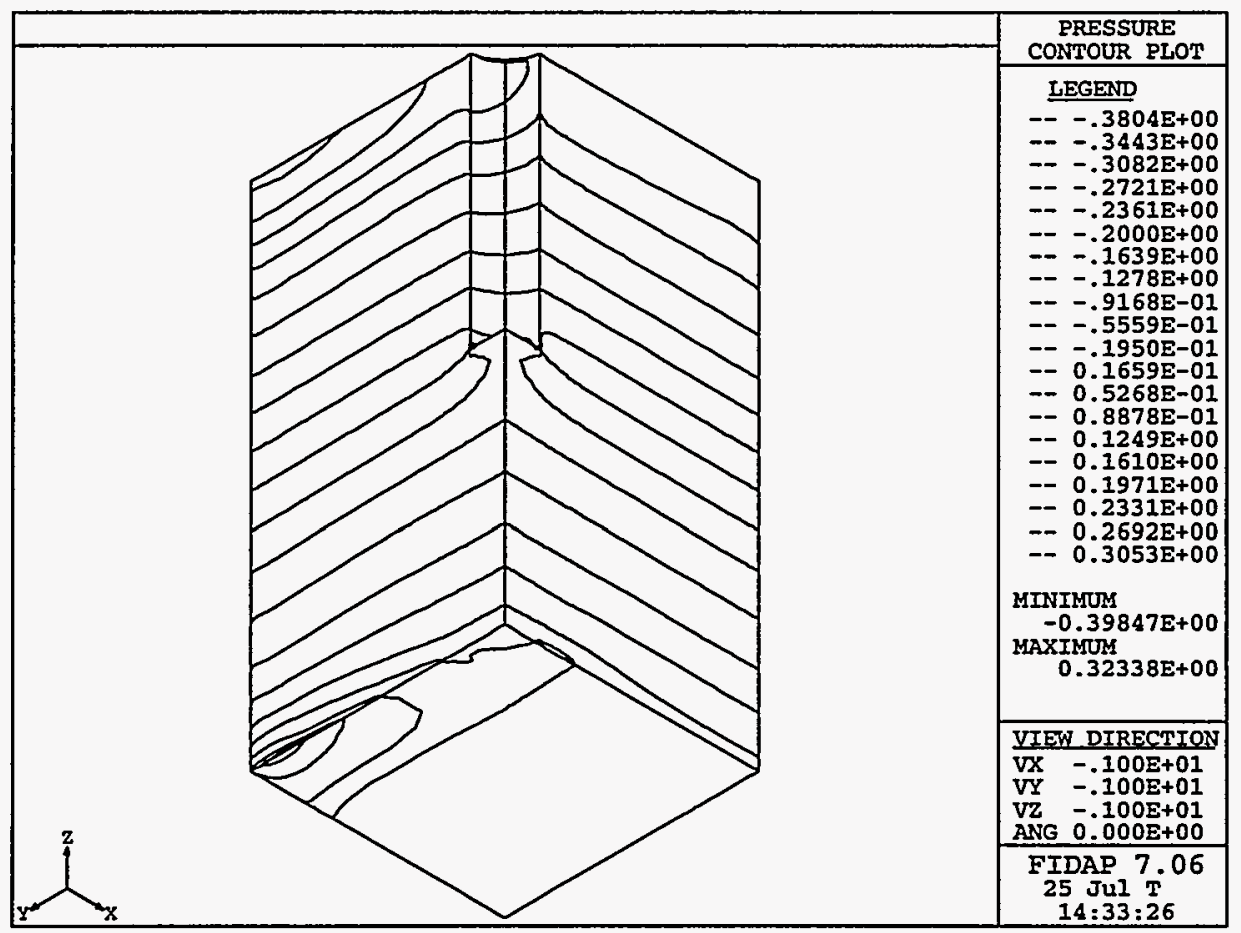

Figure 3.11 Contour plot of pressure p. 


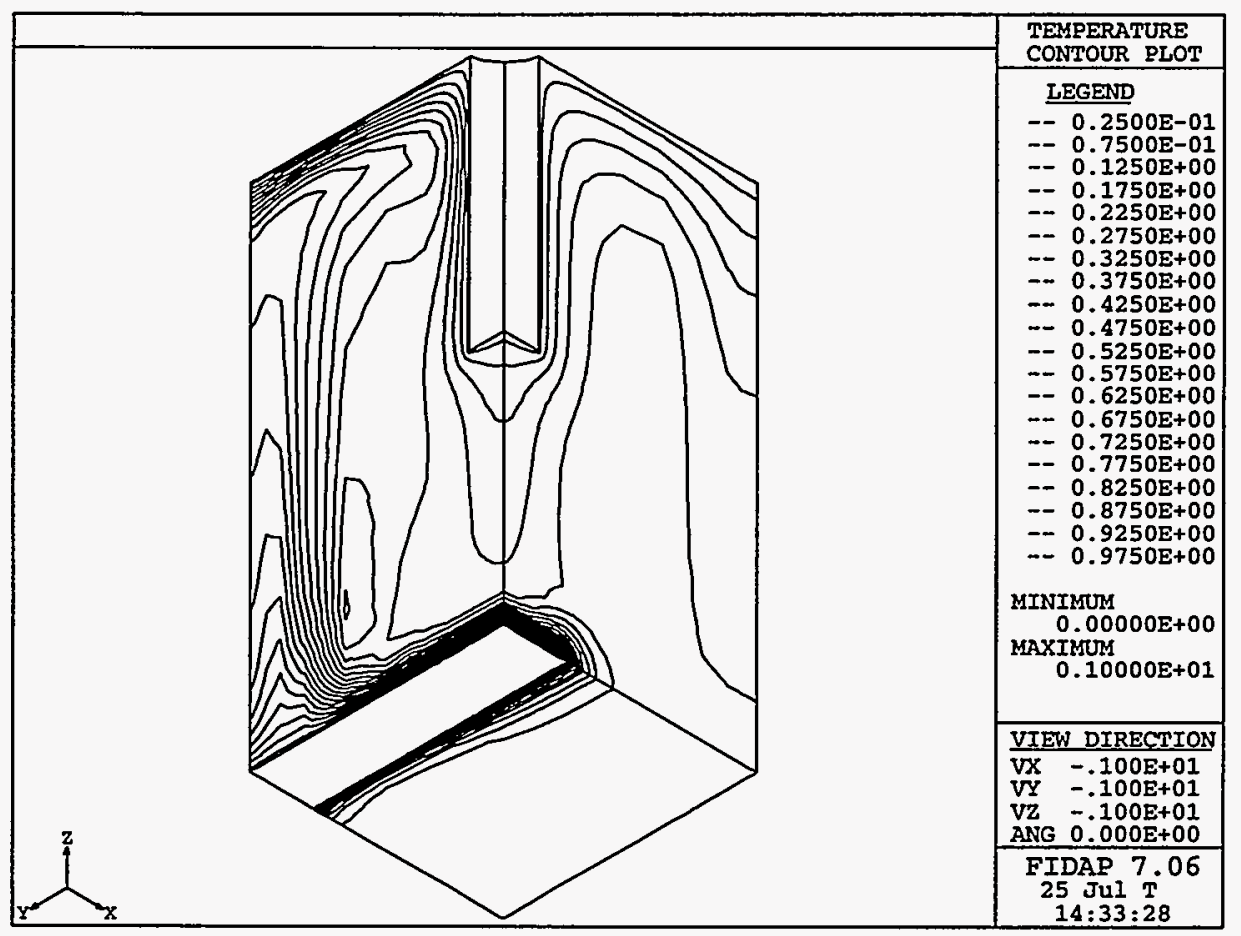

Figure 3.12 Contour plot of temperature T.

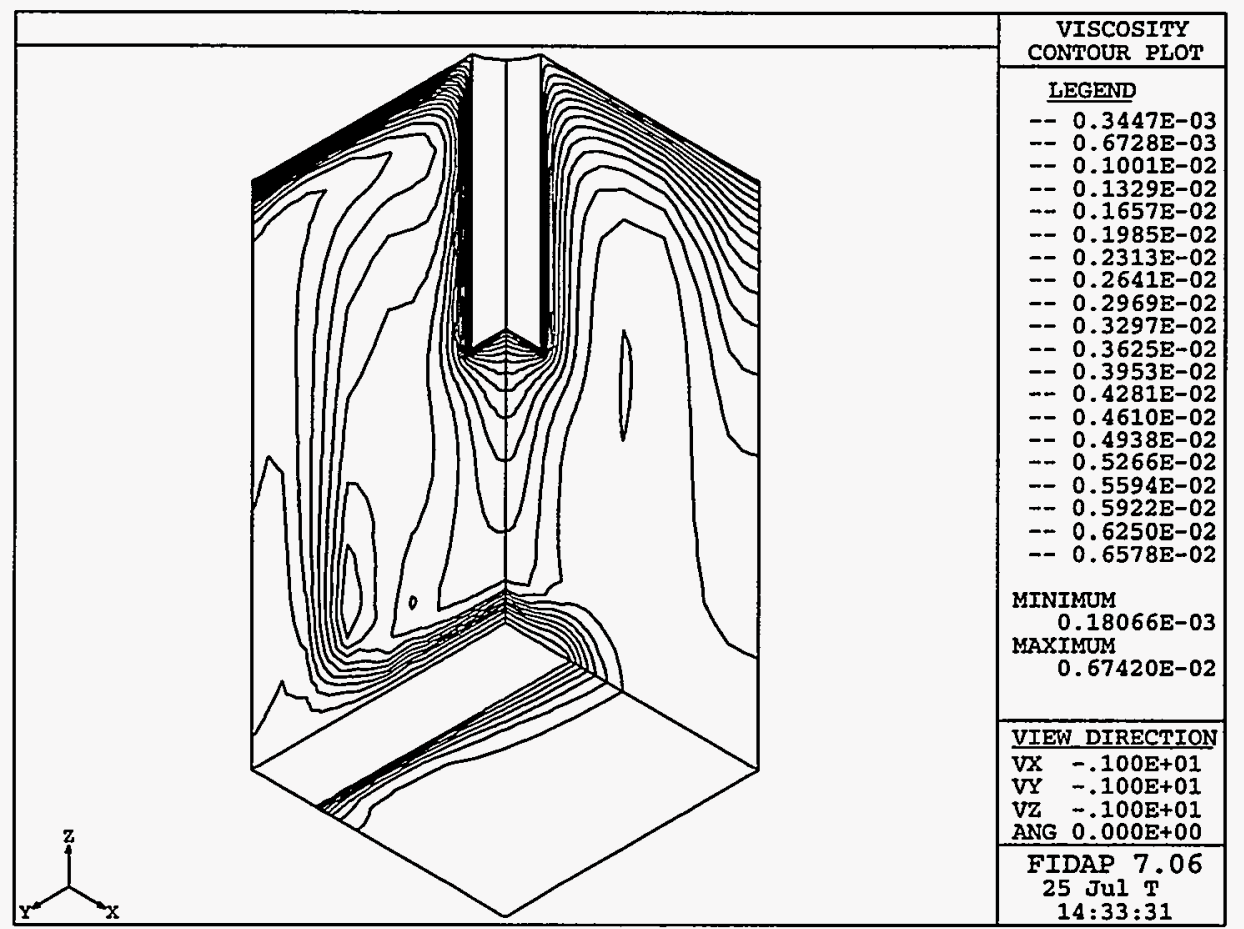

Figure 3.13 Contour plot of viscosity $\mu$. 

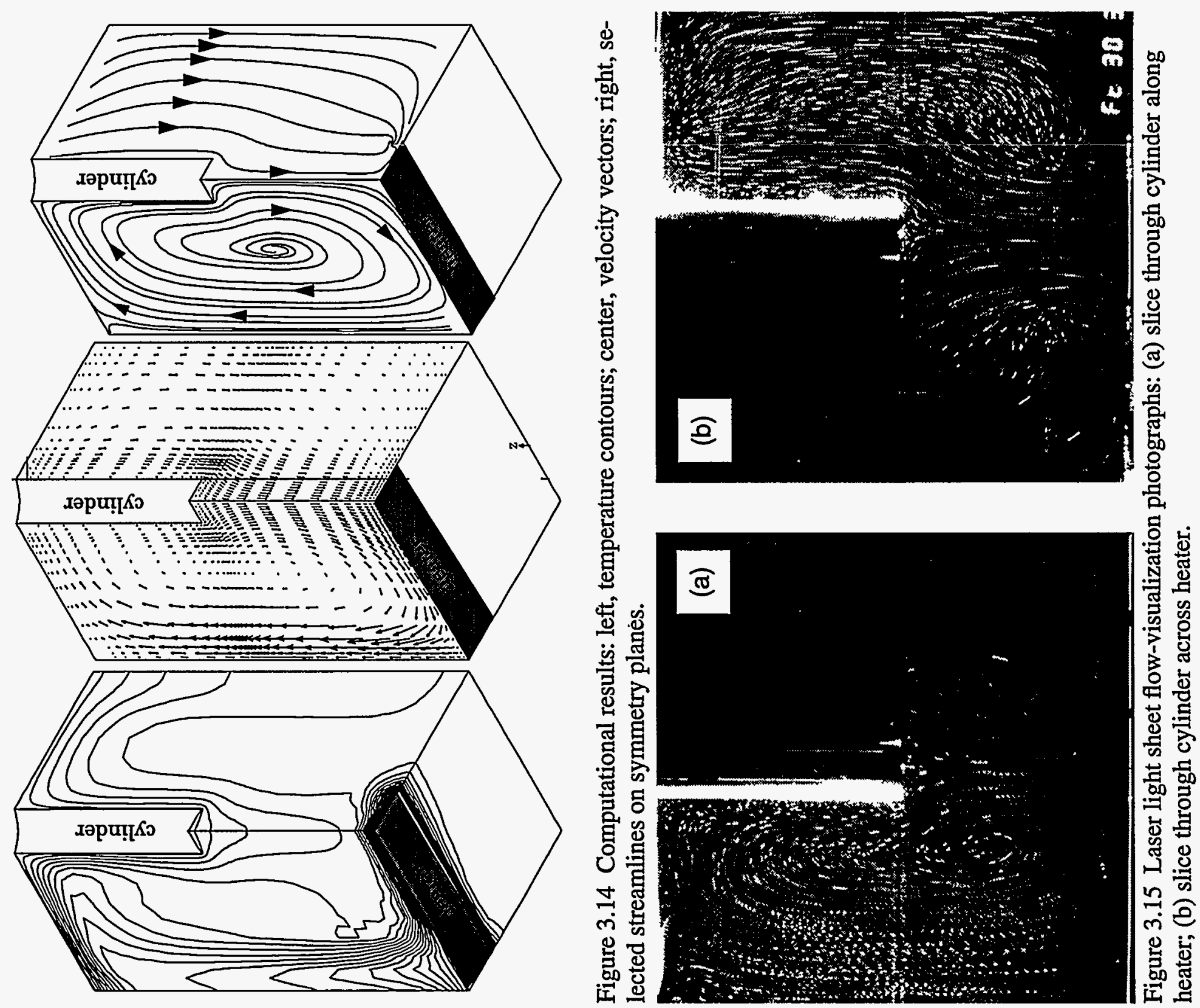
This page left intentionally blank 


\section{PHOTOREFRACTIVE OPTICS}

\subsection{Introduction}

When laser light passes through a turbulent flow, it is distorted by the density (refractive index) perturbations that are present. Although this phenomenon adversely affects the propagation of laser beams over long distances through turbulent media such as the atmosphere, it can be used to study turbulence since the perturbations of the laser beam contain information about the turbulence. Most laboratory experiments have small path lengths through the turbulent flow, so the perturbations are often quite small. This makes their detection and analysis difficult in the presence of the large, unperturbed portion of the laser beam.

Over the past few years several authors have investigated the application of nonlinear photorefractive materials (e.g., Pepper et al., 1990) to turbulence measurements, by separating the perturbations from the remainder of the beam. Robey (1990) and Robey et al. (1990) demonstrated application of the photorefractive effect by using barium titanate $\left(\mathrm{BaTiO}_{3}\right)$ as an optical filter in a turbulent channel flow experiment.

We had previously investigated the use of $\mathrm{BaTiO}_{3}$ as a high-pass temporal filter for measurement of dynamic flow fields (O'Hern et al., 1991). Barium titanate is a photorefractive crystal which can be used to generate a standing-wave Bragg grating, i.e., a self-referenced real-time hologram of an incident coherent optical wavefront (Albrecht et al., 1990). The grating has the property that it scatters, or "fans" most of the incident radiation away from the optical axis. Because the grating formation has a time dependence, dynamic components of the incident wavefront will not be scattered out of the beam, while static components will be. In this way, the crystal acts as a temporal high-pass filter. In a turbulence experiment, the steady unperturbed laser beam is fanned away, so only the perturbed signal (containing the turbulence information) is transmitted and recorded for analysis. These effects have been previously demonstrated (Robey, 1990, Robey et al., 1990, O'Hern et al., 1991, and Papamoschou et al., 1993). The goal of the present work was to better quantify, calibrate, and demonstrate the technique.

This work has highlighted the capability of $\mathrm{BaTiO}_{3}$ as a significant visualizer of turbulent vs. steady-state flow. However, we have identified two potentially significant limitations in that (1) it visualizes refractive index gradients, not absolute phase change, as desired and as reported in at least one reference, and (2) it appears to visualize refractive index gradients differently depending upon their orientation relative to the crystal fanning orientation.

We demonstrated that the $\mathrm{BaTiO}_{3}$ photorefractive effect visualizes wavefront gradients, but not absolute phase variations in an optical field. It had been desired that the effect could be used as a single-beam self-referencing interferometer, in which the visualized flow would represent changes in optical phase, not wavefront slopes. Indeed a paper by Siahmakoun and Harrer (1993) claims that absolute dynamic phase shifts are detected by the effect. We have produced an experiment 
which we believe refutes the published claim and demonstrates that changes in wavefront slopes, and not absolute dynamic phase shifts, are visualized.

We also performed a number of experiments to determine the effects of optical configuration (fanning orientation and edge orientation) on the image filtered by the $\mathrm{BaTiO}_{3}$. Sensitivity of the photorefractive technique to orientation of the fanning direction of the crystal was demonstrated. The implication is that $\mathrm{BaTiO}_{3}$ is sensitive to optical disturbances in one preferred gradient direction only, but does not visualize disturbances isotropically. Isotropic visualization is needed for unbiased measurement of turbulent flow fields. Furthermore, we raise concerns about whether a one-to-one correspondence can be established between a flow field and its visualized image due to mixing of the wavefront within $\mathrm{a} \mathrm{BaTiO}_{3}$ crystal.

\subsection{Experimental Investigations}

In this program we have attempted to demonstrate the use of a photorefractive crystal as a visualizer of optically refractive turbulent flow. We had also expected to draw quantitative data from the visualization, but this objective was not met for the reasons detailed below. There are two basic modes of operation using this technique. The first involves direct measurement of the turbulent power spectrum, while the second is a photorefractive schlieren. In both techniques, the $\mathrm{BaTiO}_{3}$ crystal scatters out (fans away) all wavefront components which are static to within some time scale, allowing only the fluctuating turbulent flow component to transmit through the remainder of the optical system.

We have demonstrated the operation of $\mathrm{BaTiO}_{3}$ using a cubic crystal with the faces cut at 45 degrees to the crystal "C" axis. Prior work (O'Hern et al., 1991) used crystals with the face cut normal to the " $\mathrm{C}$ " axis, which required tilting the crystal at a 45 degree angle relative to the input laser beam in order to observe the photorefractive effect. The tilt generated considerable astigmatism in converging wavefronts. Our current arrangement minimizes this effect.

\subsection{Experimental Results}

\subsubsection{Turbulent Power Spectral Density Measurements}

The power spectral density (PSD) diagnostic depends upon the property that a lens forms the twodimensional Fourier power spectrum of an optical disturbance field at the lens focal plane. In the Fourier plane, one can then view the spatial frequency distribution of the optical disturbances. One of the problems with viewing the Fourier plane is that the nonzero spatial frequency components are usually weak and therefore dwarfed by the intensity of the zero spatial frequency (DC) component. By including the $\mathrm{BaTiO}_{3}$ crystal into the beam path, the $\mathrm{DC}$ component of the beam is removed by fanning away from the optical axis, and the higher spatial frequency components can be better observed (Robey, 1990, Robey et al., 1990). 
The experimental arrangement for the PSD setup is shown in Fig. 4.1. A Spectra-Physics 165 argon-ion laser operating at $514.5 \mathrm{~nm}$ was used as the optical source. The polarization of the laser was oriented in a vertical plane. The laser beam was expanded to $50 \mathrm{~mm}$ in diameter and directed through a flow cell where refractive disturbances were introduced. The optical configuration was modeled on ZEMAX, an optical design program, to establish that the design was capable of high quality optical imaging. The beam was directed into a $500 \mathrm{~mm}$ focal length doublet which then focused the beam to a point image. This plane constituted the "Fourier" plane, i.e., where the optical Fourier transform of the flow disturbance is formed. The $\mathrm{BaTiO}_{3}$ crystal was positioned at this location with an orientation such that the resulting fanning was in the vertical direction. The transmitted light was expanded into a $125 \mathrm{~mm}$ focal length triplet relay lens which then directed the beam to a 1/2" Pulnix model TM745 charge-coupled device (CCD) based video camera. The camera was positioned at the image Fourier plane. Between the relay lens and the CCD camera neutral density filters could be positioned to attenuate the beam as needed. The video camera was coupled to an Imaging Technology VFG 100 frame grabber installed in a 386-based PC. The frame grabber permitted real-time observation of the CCD image, or up to four video frames to be captured in succession and stored in memory.

We demonstrated power spectrum measurements using a cold jet directed across the test section region. Fig. 4.2 illustrates the result. For the static case (regions of uniform density), the beam becomes nearly invisible, while in the dynamic case (regions of large density gradient), the power spectral density distribution becomes visible. In instances where we removed the photorefractive crystal from the optical train, the image of the power spectral density is swamped by the DC component of the signal. A static stray reflection is evident in all photos.

We attempted to calibrate the system for quantitative measurement of the PSD but quickly ran into difficulties. The PSD is actually a complicated relationship between the magnitude and spatial scale length of the phase disturbances (Papamoschou et al., 1993). Measuring the spatial scale of the disturbance would be straightforward since the width of the PSD is proportional to the maximum spatial frequency of the turbulent scale length. However, the Fourier transform is not a one-to-one relationship in phase because the phase term is a complex exponential. These PSD experiments emphasized that the major challenge remains quantification and calibration of the PSD image such that turbulence information can be extracted in order to evaluate the structures existing in the flowfield.

\subsubsection{Temporal Schlieren Visualization}

The temporal schlieren method, like the classical schlieren technique, visualizes spatial variations in the flow as an intensity variation in the schlieren image. In a typical implementation, a lens is used to image the flow field, which is illuminated by a collimated coherent laser wavefront, onto an image plane. At the focal (Fourier) plane of the lens, which lies between the lens and the image plane, the classical schlieren method uses a knife edge or slit to block optical gradients of specific values (Merzkirch, 1987). Spatial gradients in the flow field then appear as specific regions of light and dark, regardless of temporal history. A high quality wavefront must be transmitted through the 
system with no temporal disturbance in order to present a nominal dark field view of the flow field. With the $\mathrm{BaTiO}_{3}$ method, the crystal replaces the knife edge. Instead of blocking out gradients of a specific slope, the crystal scatters out all wavefront components which are static to within some time scale. Then, at the image plane, only the fluctuating turbulent flow components are viewed, even if large static optical disturbances are present. This yields, in effect, a dynamic, real-time holographic schlieren system.

The setup is shown in Fig. 4.3. The only difference between this configuration and the PSD configuration is that the CCD camera is positioned at a point where the phase disturbance plane is imaged. The visualized turbulent flow is shown in Fig. 4.4. In this example flowing $\mathrm{CO}_{2}$ gas from a nozzle is visualized. The illustration shows how the visualized image flow can be combined with image processing to subtract out the background which was not completely extinguished by the crystal. Not shown is the image produced with no crystal present. In this case, the turbulent flow could not be visualized at all. Additional examples of photorefractive schlieren images are shown in O'Hern et al., 1991.

We demonstrate that the crystal is sensitive to dynamic effects only, and not static refractive effects in Fig. 4.5. In this instance, a phase object consisting of a heated soldering iron is placed in the field of view. When the soldering iron is allowed to generate a laminar heat flow in the surrounding air as in (A), the phase disturbance is invisible, but when the air is disturbed, as by introducing a slight cross current (B), the vertical stream because visible. View (C) is an image of image (A) subtracted from image (B) using image processing.

\subsubsection{Orientation Effects on Flow Visualization}

A number of experiments were conducted to determine the effects of fanning orientation and edge orientation on the image produced by the $\mathrm{BaTiO}_{3}$ technique. The sensitivity of the photorefractive technique to orientation of the fanning direction of the crystal was initially noticed when a compressed-gas jet was directed horizontally then vertically with the same optical setup (Fig. 4.6). While the horizontal jet imaged strongly through the crystal, vertical venting of the jet produced very little dynamic response within the crystal. This directionality effectively introduces a bias into the image of any particular flow because the crystal will preferentially image gradients in a direction perpendicular to the fanning direction of the steady part of the optical signal. The strength of the directional sensitivity was quantified by comparing gray scale histograms for the images of the jet oriented horizontal and vertical, yielding significantly different distributions (Fig. 4.6C).

We repeated the demonstration with a well-controlled helium jet flow field. Figure 4.7 illustrates the effect. A low-pressure ( 5 psi) jet flow was directed across the test section perpendicular and parallel to the fanning direction. The perpendicular flow was visualized at a significantly brighter intensity than the parallel flow. Since the gradients in a flow are significantly greater in the direction perpendicular to the flow direction, we concluded that the photorefractive visualization is most sensitive to gradients in the fanning direction. The conclusion is that, like classical schlieren techniques, the photorefractive technique is sensitive to gradients in one orientation only. 


\subsubsection{Sensitivity to Wavefront vs. Slope Disturbances}

We have demonstrated that the $\mathrm{BaTiO}_{3}$ photorefractive effect visualizes wavefront gradients, but not absolute phase variations in an optical field. It had been desired that the effect could be used as a single beam self-referencing interferometer, in which the visualized flow would represent changes in optical phase, not wavefront slopes. Siahmakoun and Harrer (1993) claim that absolute dynamic phase shifts are detected by the photorefractive effect. We believe the following demonstration refutes the published claim by showing that changes in wavefront slopes, and not absolute dynamic phase shifts, are visualized. We contend that Siahmakoun and Harrer observed an effect due to changes in the polarization state of light due to birefringence, and not due to absolute phase change. In our experiment, we arranged two mirrors which share the aperture illuminated by the collimated beam (Fig. 4.8). One mirror was attached to a PZT translator driven by a ramped high voltage signal. This drove the mirror axially in "piston" through several waves of optical path change. The other mirror was held stationary. Were the crystal sensitive to absolute dynamic phase changes, one would observe in the image plane a modulated intensity across the aperture of the moving mirror, and no modulation in the image of the static mirror. In fact we observed no significant modulation in either mirror (Fig. 4.9), nor were there any substantial differences in images if one or another beam was blocked either before or after the crystal. However, we could observe some stray moving interference fringes, probably due to scattered light off of various surfaces in the crystal. Figure 4.10 illustrates that the extent to which extraneous temporally modulated fringes were observed was a function of how much overlap existed between the two beams inside the crystal. Beams overlapping within the crystal exhibit considerably more temporally modulated fringes than outside the crystal. This phenomenon alone is an indicator that the $\mathrm{BaTiO}_{3}$ crystal as a flow visualizer is a complex effect and may be difficult to refine as a quantitative diagnostic.

\subsubsection{Effect of Apertures}

Obscurations placed across the object field contributed an extraneous glow at edges of the aperture. The observed glow was strongly dependent upon the orientation of the diffractive edge relative to the fan direction within the crystal and to the power of the incident beam entering the crystal. If a diffracting edge lay parallel to the fan direction, the effect was weak or nonexistent (Fig. 4.11). However, an edge perpendicular to the fan direction appeared to glow strongly, with an intensity dependent upon how much of the aperture was blocked (Fig. 4.12). This is additional evidence that there is an orientation effect associated with the $\mathrm{BaTiO}_{3}$ techniques.

\subsubsection{Temporal Transmission Measurements}

The temporal crystal fanning response is highly dependent upon incident optical power density levels within the crystal. It is also dependent upon the residual grating remaining in the crystal from previously recordings. This effect made it difficult to characterize the crystal temporal bandpass 
response, i.e., the $\mathrm{BaTiO}_{3}$ temporal filter "settings." We did find that by repeatedly erasing the previously recorded Bragg grating with an incandescent, incoherent light source, we could obtain repeatable temporal extinction profiles which were dependent upon the length of the erasure light exposure. Figure 4.13 shows relative crystal extinction vs. time after a poling operation of the specified duration on a crystal which had achieved maximum extinction on a prior exposure. The time dependence of extinction appears to be approximately a negative exponential whose initial transmission is a function of the initial poling time. The peak transmission vs. poling time is replotted in Fig. 4.14, and can be seen to be approximately logarithmic in behavior.

\subsection{Photorefractive PIV}

Traditional PIV data analysis (see Chapter 2) is time-consuming due to the computer time required to perform digital autocorrelations of the image fields. Fast optical autocorrelations are possible using Fourier lenses and photorefractive materials: in this case the computer image processor need only read the location of the autocorrelation peaks stored in the photorefractive crystal rather than compute the digital autocorrelation (Buchhave and Jakobsen, 1990; Marrakchi et al., 1991). Such an increase in analysis speed would make PIV much more useful for flow measurements, especially those with transient conditions and/or a large test matrix.

A photorefractive PIV (PPIV) system had been proposed for investigation as part of this program. However, due to the image degradation limits cited in the previous sections, this portion of the work was not attempted.

\subsection{Summary}

$\mathrm{BaTiO}_{3}$ has been demonstrated as a significant visualizer of turbulent vs. steady-state flow. However, we have identified two potentially significant limitations in that it (1) appears to visualize gradients differently, depending upon the relative orientation of the gradient to the crystal fanning orientation, and (2) visualizes refractive gradients, not absolute phase change, as reported in at least one reference. The desired quantification of photorefractive measurements proved to be very difficult for the reasons discussed above. 


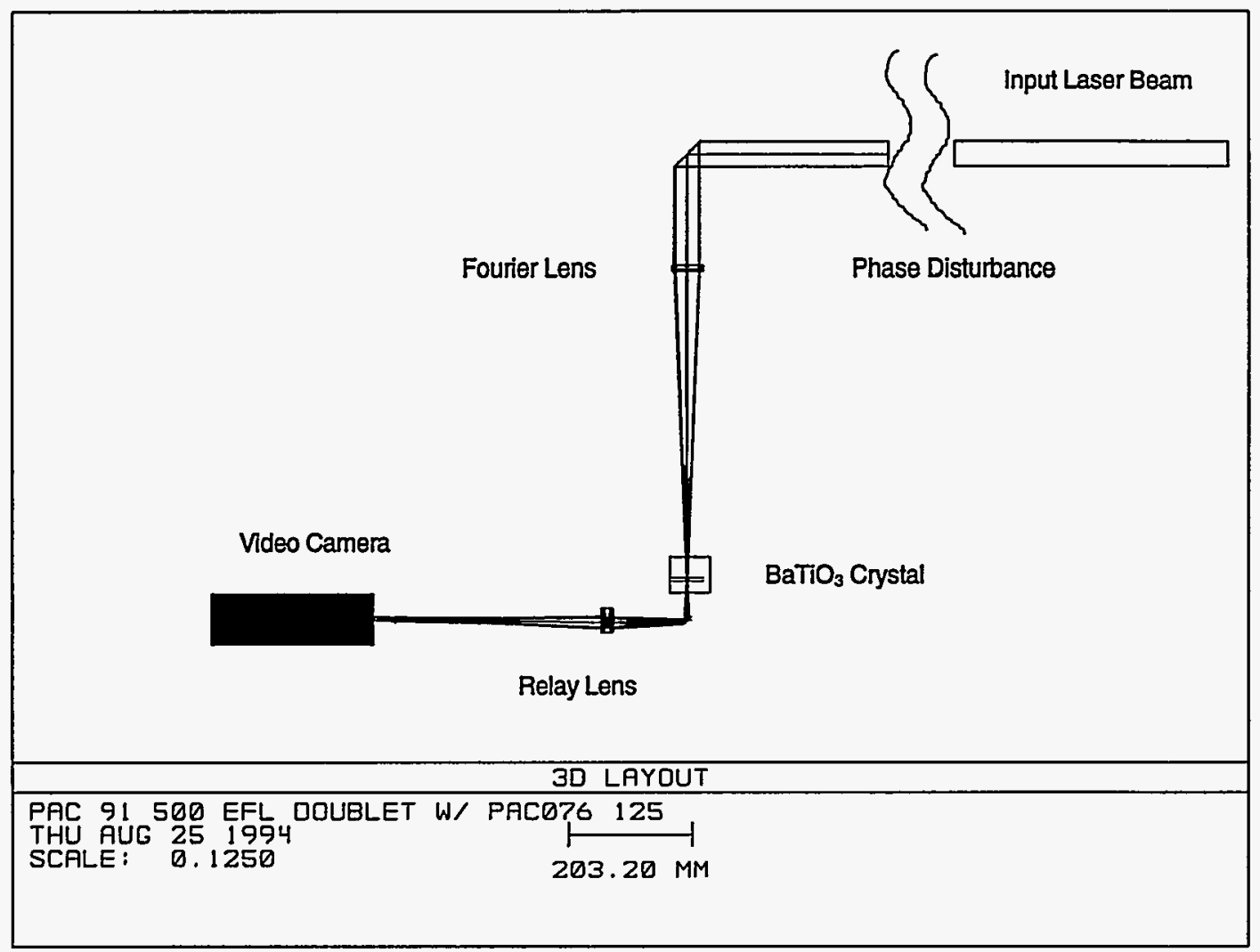

Figure 4.1. Schematic layout of optical system for power spectral density (PSD) measurements. Fourier lens $500 \mathrm{~mm}$ focal length, relay lens $125 \mathrm{~mm}$ focal length. Video camera images Fourier plane of relay lens.
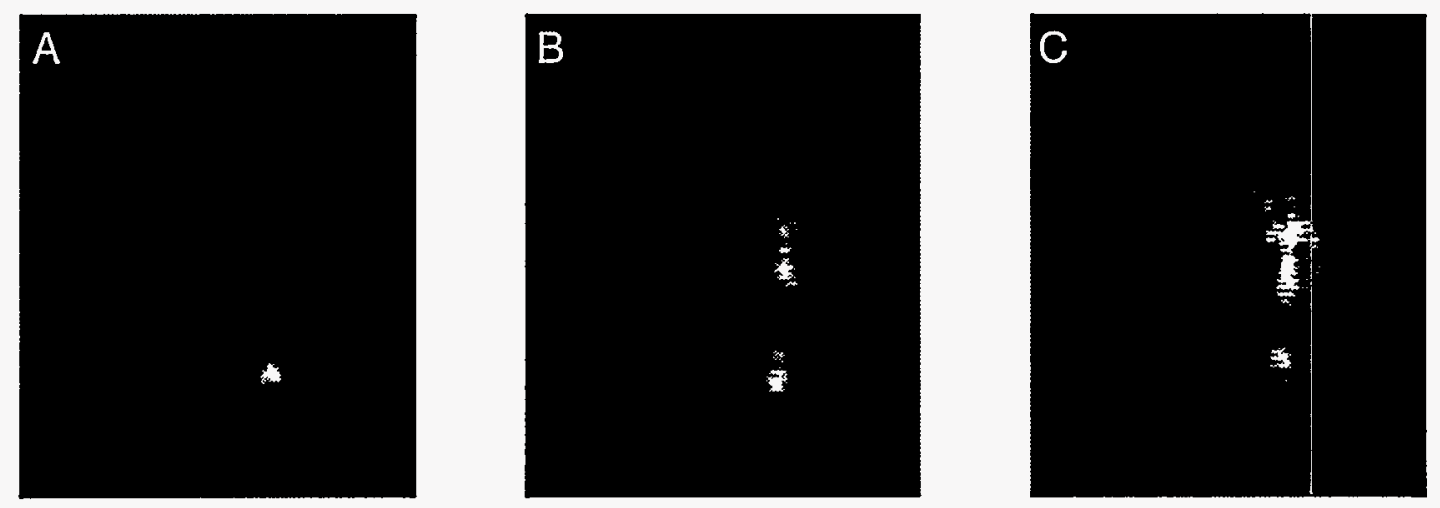

Figure 4.2. Turbulent power spectral density (PSD) measurements. (A) Static background image (no flow). (B) Gas jet: low-flow conditions. (C) Gas jet: high-flow conditions. 


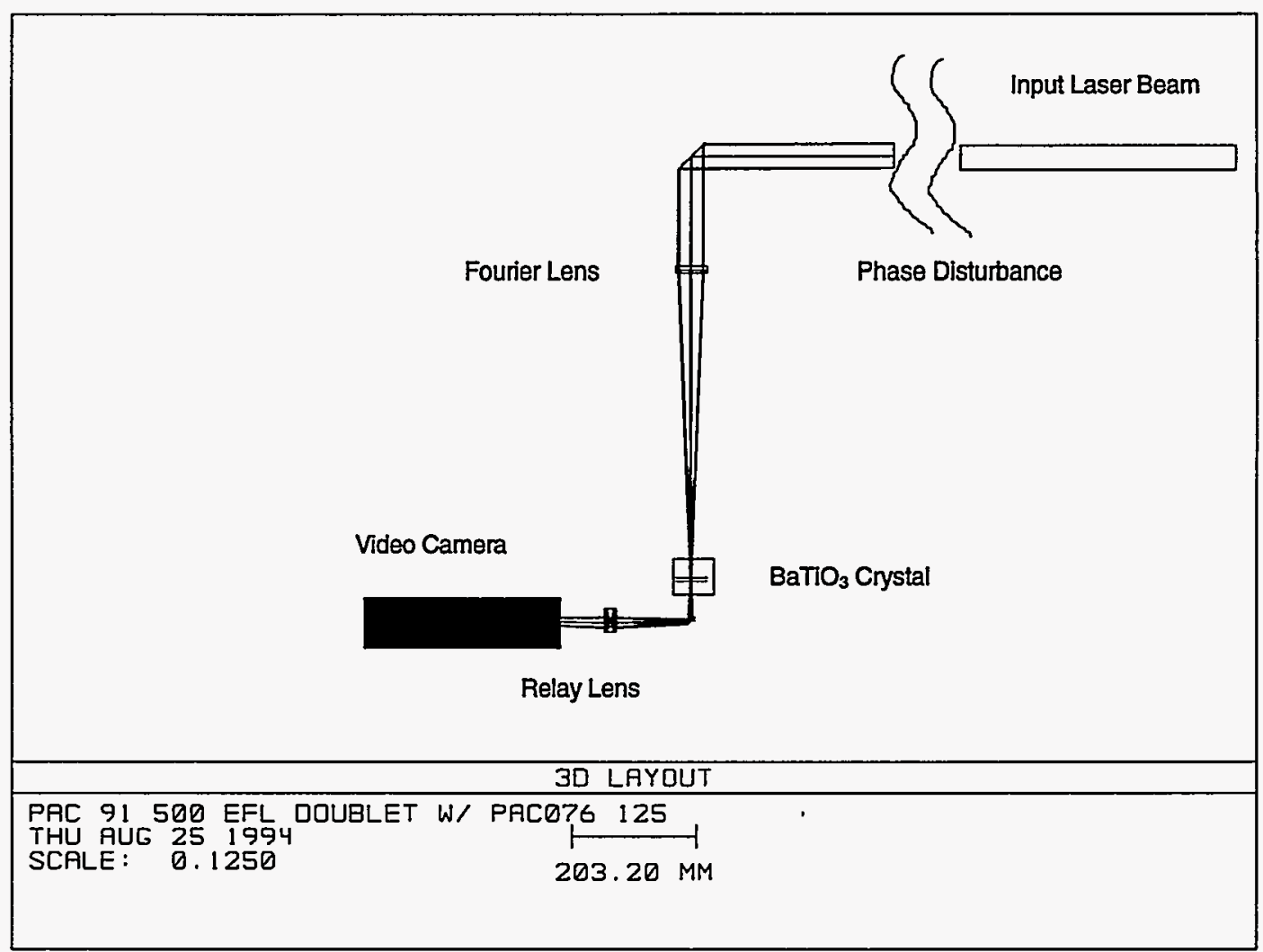

Figure 4.3. Schematic layout for photorefractive schlieren technique. Video camera images the phase disturbance plane.
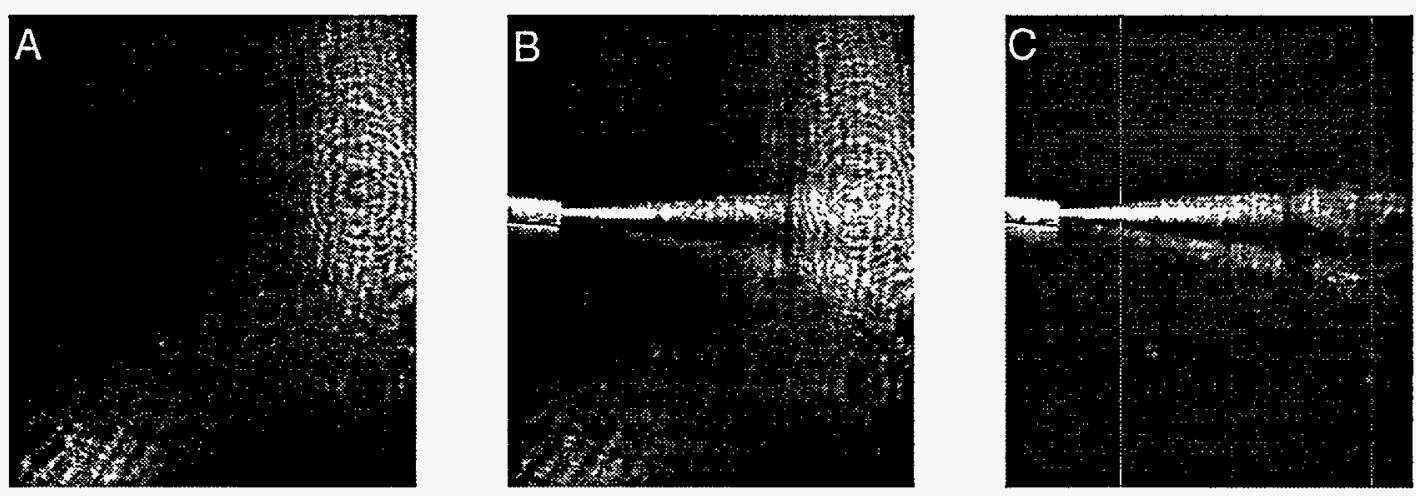

Figure 4.4. Photorefractive schlieren flow visualization with $\mathrm{BaTiO}_{3}$. (A) Background field, no flow. (B) $\mathrm{CO}_{2}$ jet flow field. (C) Jet flow field with background subtracted and image processed. 

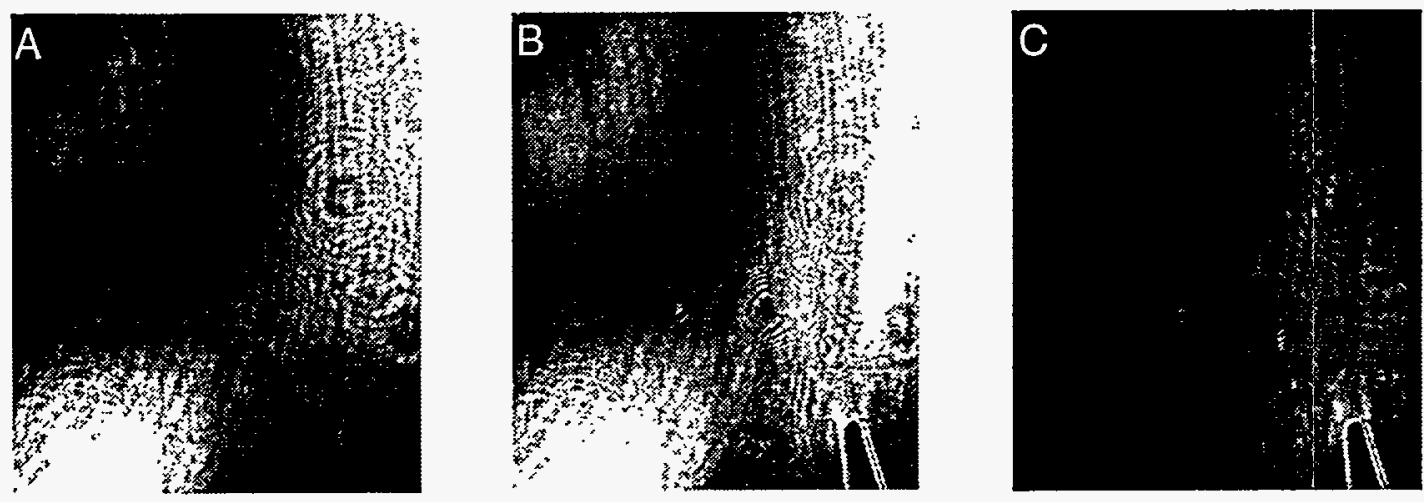

Figure 4.5. Effect of flow perturbation on $\mathrm{BaTiO}_{3}$. Test object is hot soldering iron. (A) Static laminar flow. (B) Perturbed flow field (air currents). (C) Perturbed flow field with static flow subtracted. 


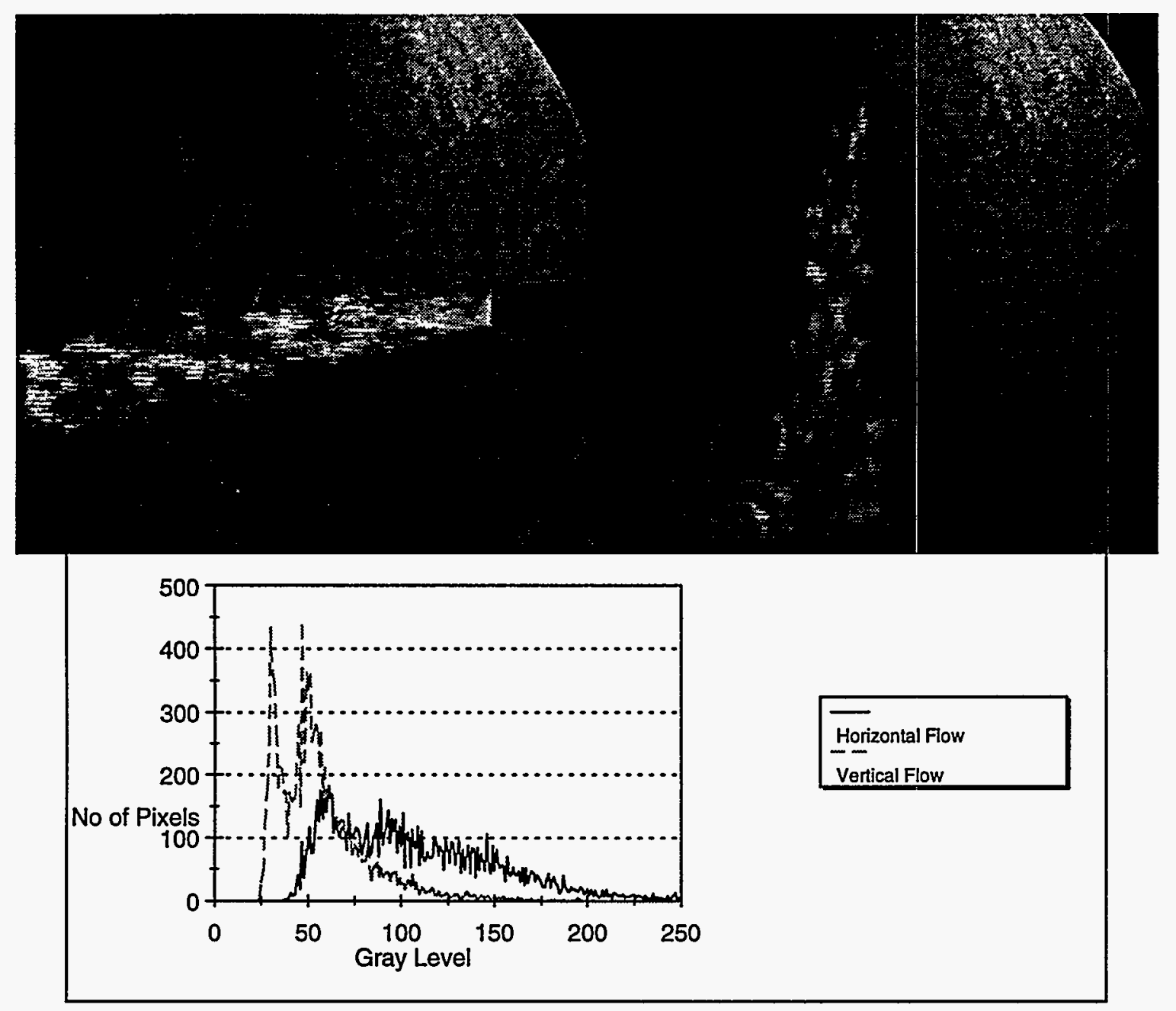

Figure 4.6. Orientation dependence of a turbulent $\mathrm{CO}_{2}$ jet in air. (A) Horizontal flow. (B) Vertical flow. (C) Intensity histograms. 


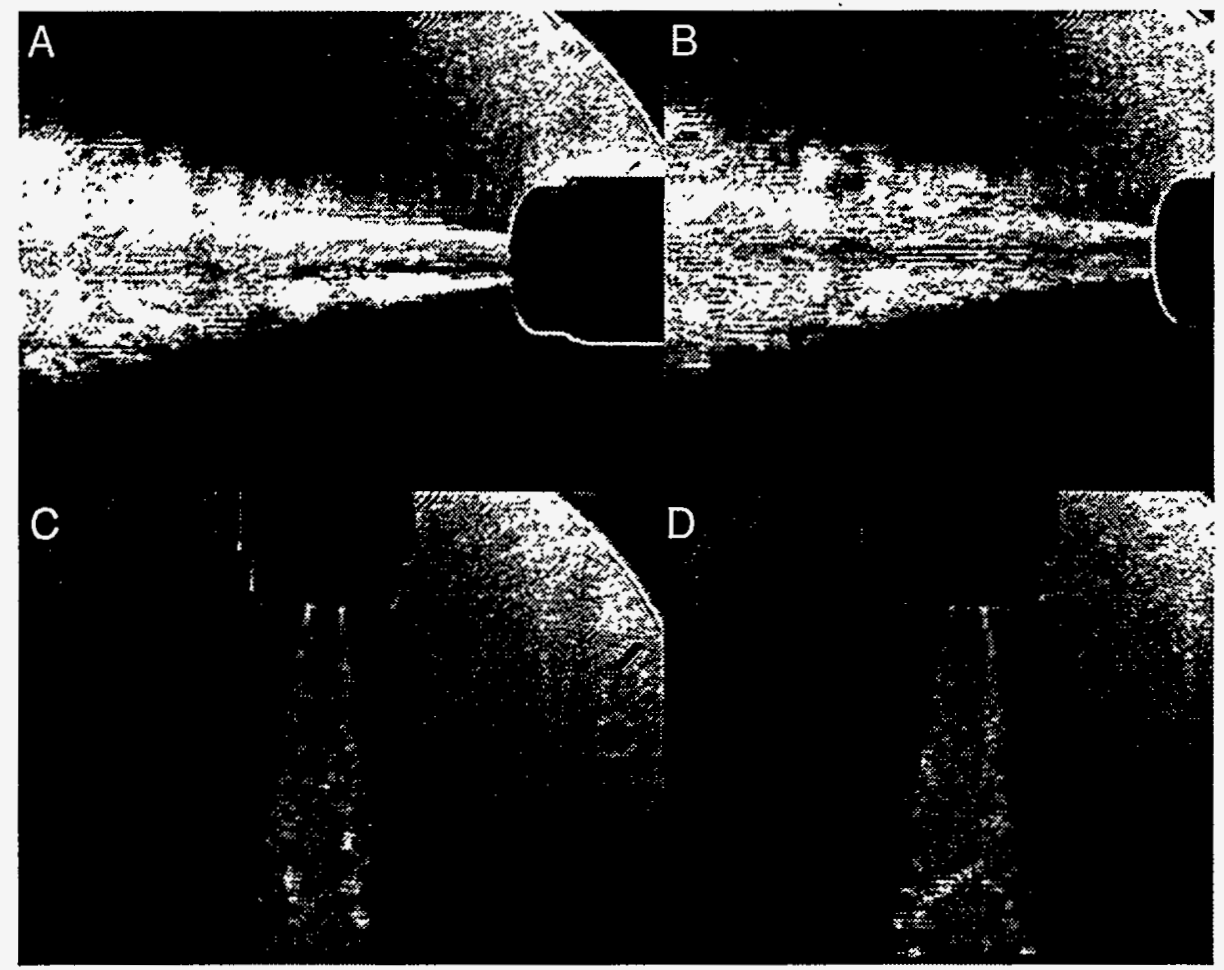

Figure 4.7. Dependence of photorefractive effect on gradient orientation. (A, B) Cross flow. (C, D) With flow. Crystal fanning is in vertical direction.

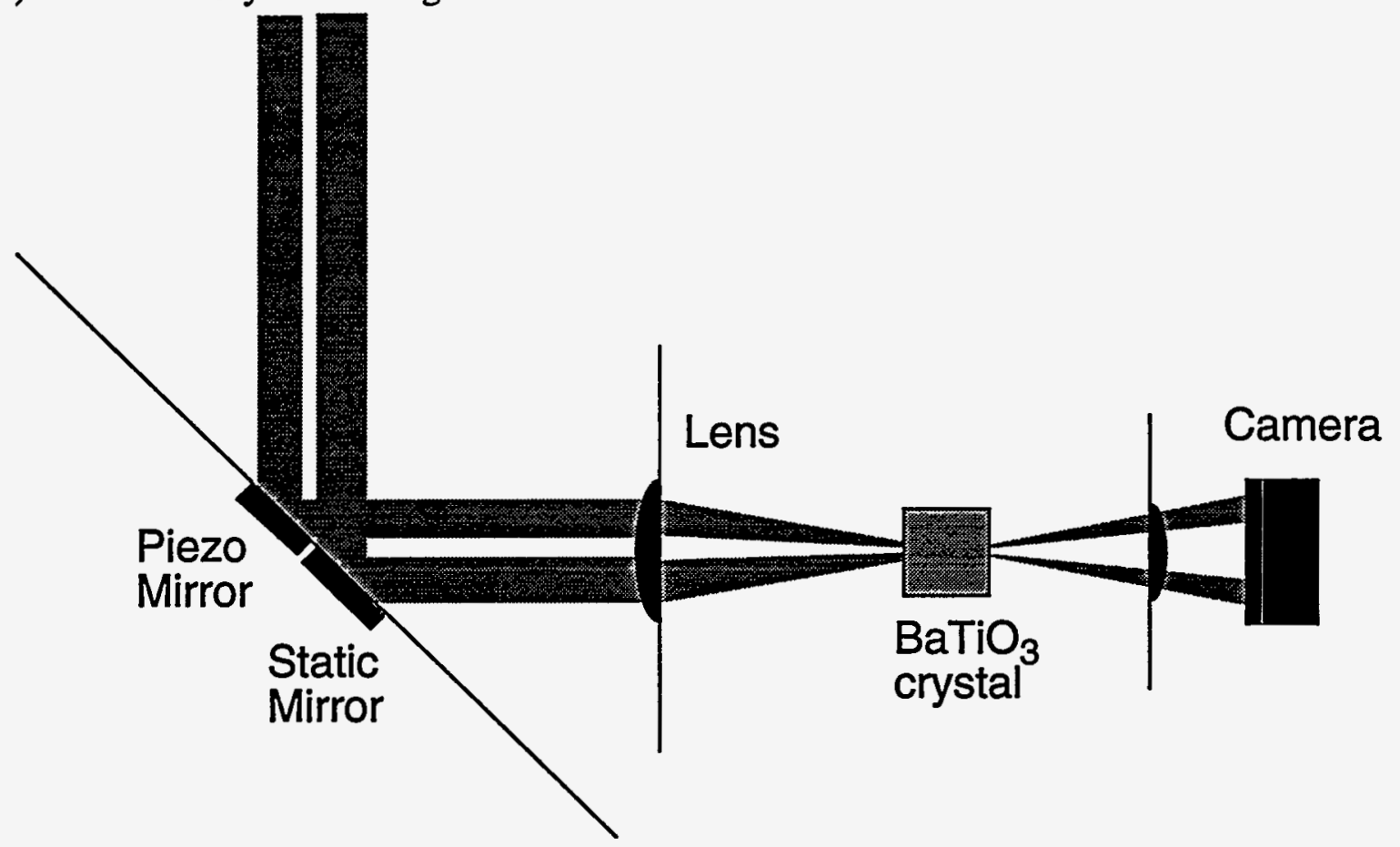

Figure 4.8. Schematic of phase sensitivity test setup. 


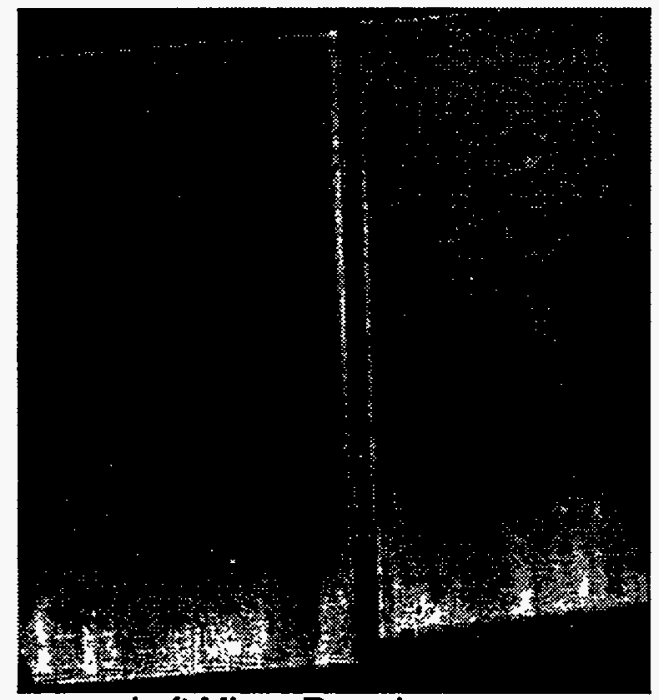

Left Mirror Ramping

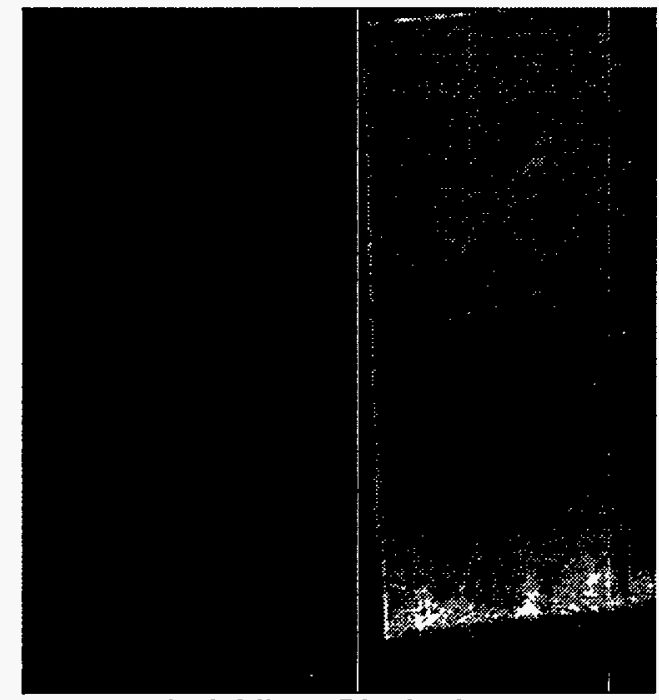

Left Mirror Blocked

Figure 4.9. Phase shift test.

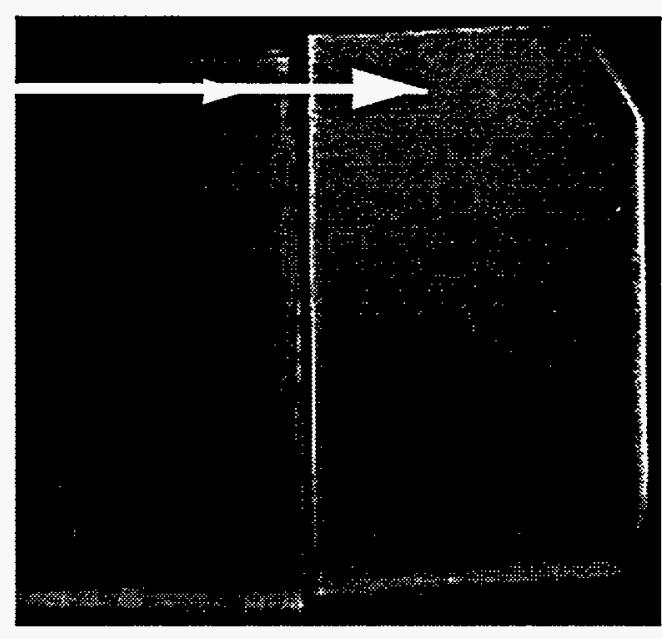

Beam Overlap Outside Crystal

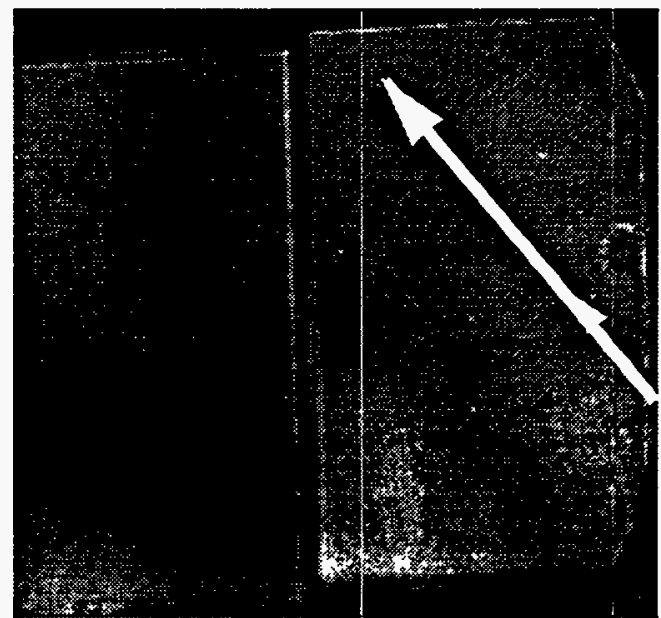

Overlap Within Crystal

Figure 4.10. Effect of cross-coupling beams within crystal. The arrows point to regions of temporally modulated fringes. 


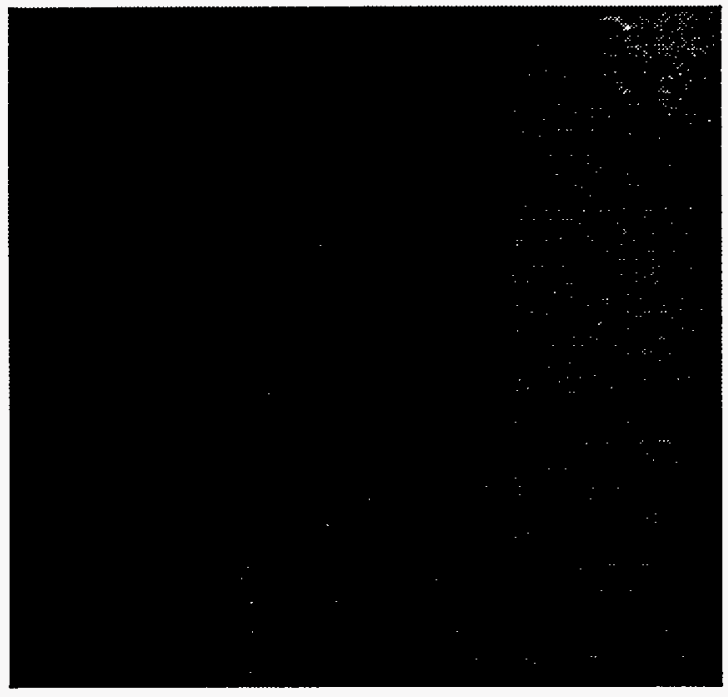

Vertical Strip Across Aperture

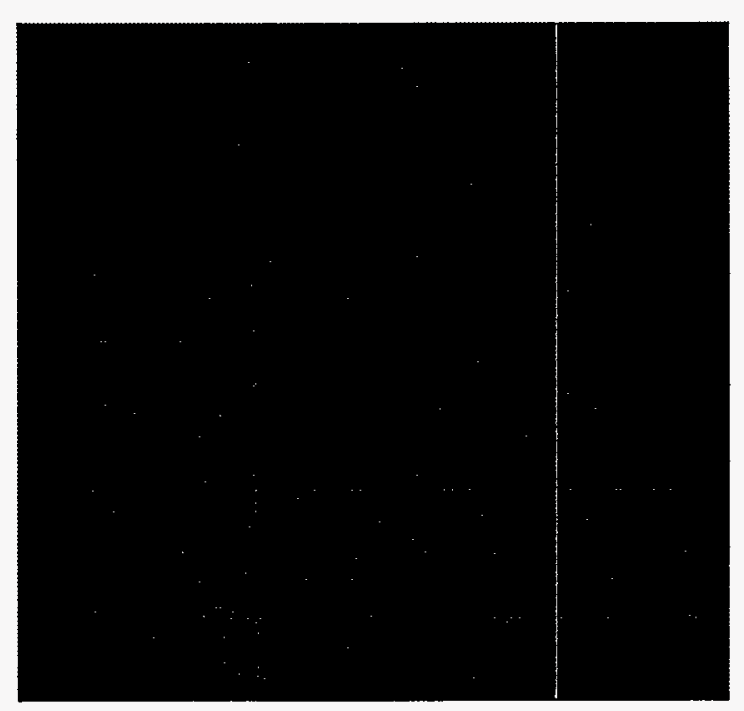

Right 2/3 Aperture Blocked

Figure 4.11. Effect of a vertical aperture on wavefront extinction: fanning direction vertical.

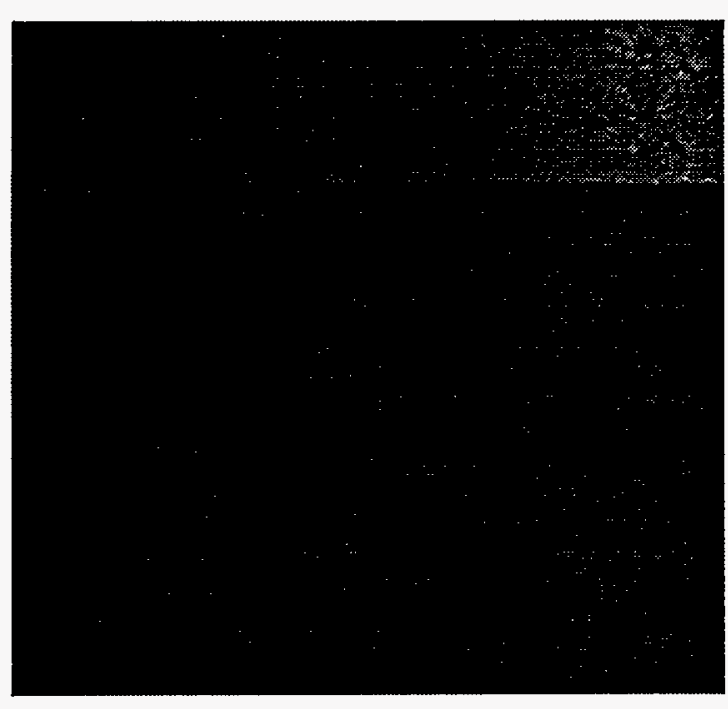

Narrow Strip Across Window

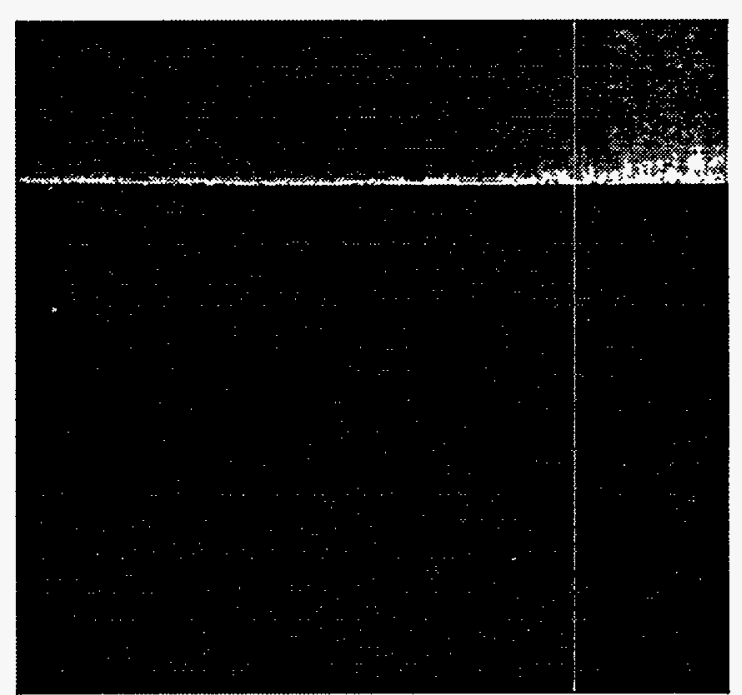

Lower $2 / 3$ blocked

Figure 4.12. Effect of a horizontal aperture on wavefront extinction: fanning direction vertical. 


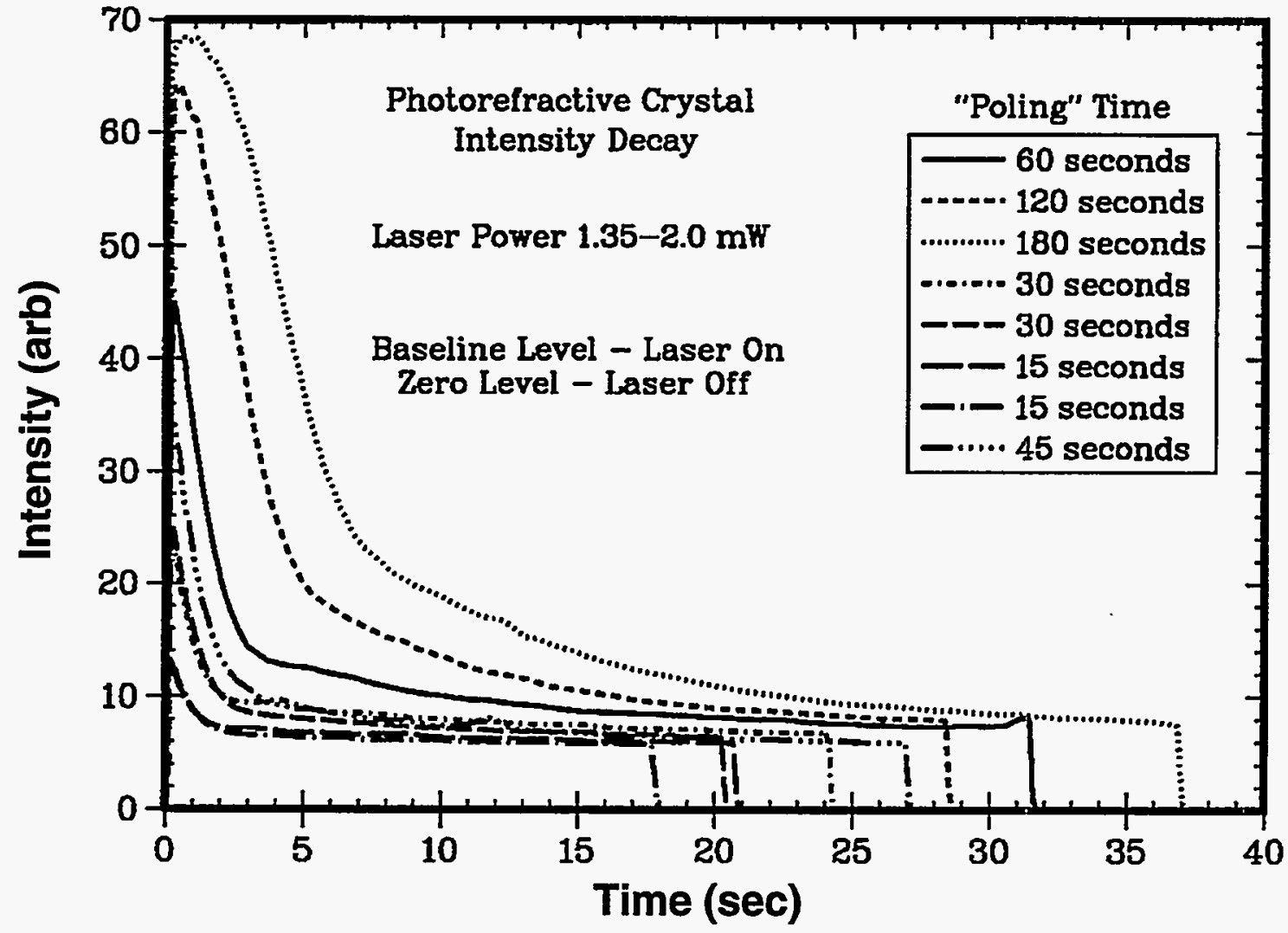

Figure 4.13. Crystal extinction vs. time for a variety of initial poling (erasure exposure) times. Incident laser power constant. 


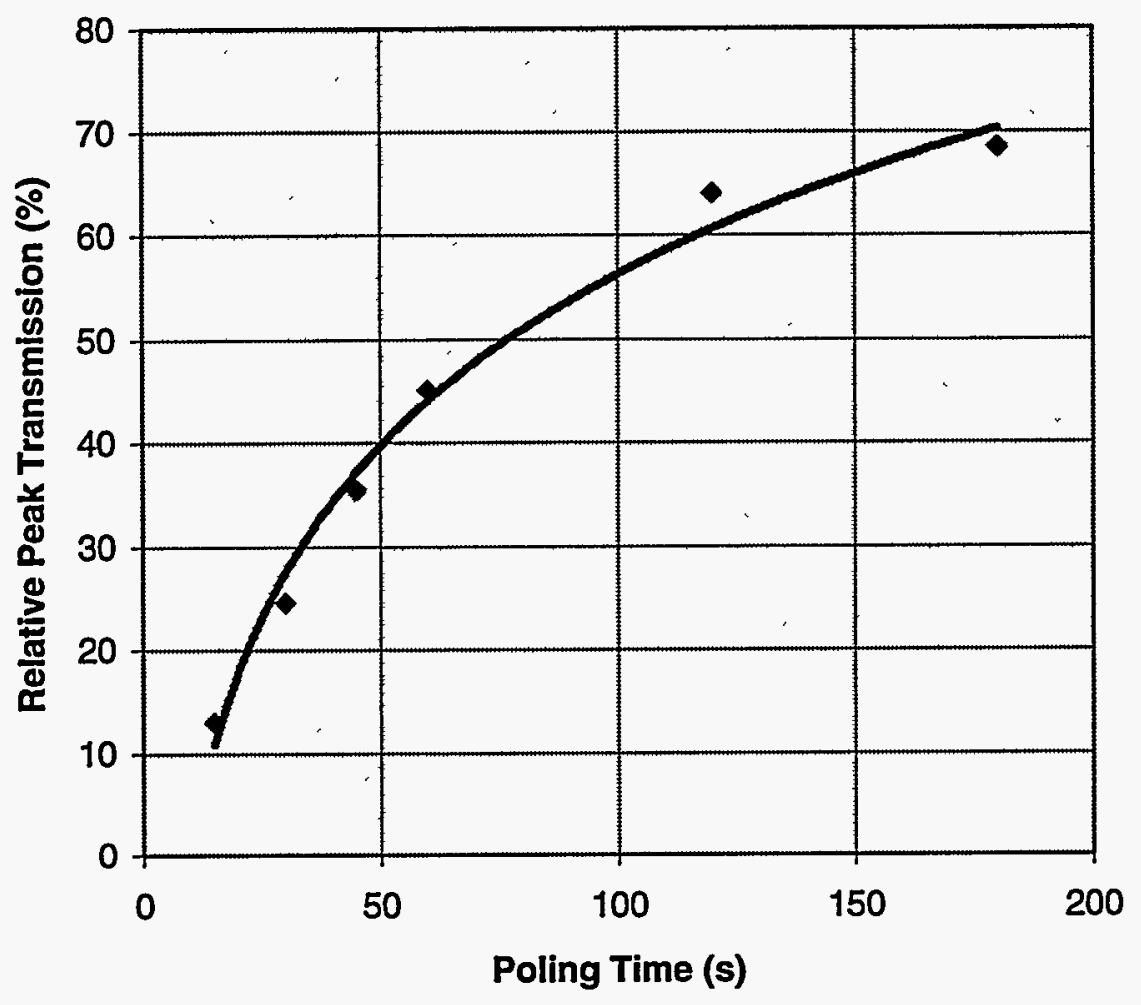

Figure 4.14. Crystal peak transmission vs. poling time. 


\section{CONCLUSIONS}

The goal of this work was to develop optical diagnostic techniques to provide Sandia with advanced flow-measurement capabilities. The first technique is Particle Image Velocimetry (PIV), in which a flow seeded with small particles is photographed repeatedly at short time intervals. Image-processing techniques are applied to find particle velocities from particle positional changes in successive frames, which permits determination of the instantaneous flow field and associated turbulence quantities. Standard PIV uses a light sheet to illuminate a plane of the flow for 2-D measurements, while either volume illumination and multiple cameras, or multiple-exposure holography, are used for 3-D PIV. The second technique uses nonlinear photorefractive optical crystals to measure turbulence quantities more directly. A laser beam passed through the flow acquires a small perturbation signal related to the turbulence, which is superimposed on a large steady informationless signal, rendering interpretation difficult. The laser beam is then passed through one or more crystals to remove most of the steady portion, thereby greatly improving the signal-to-noise ratio.

The two-dimensional PIV system has been fairly well proven on two test flow configurations, a cavity flow and a thermal convection experiment. Both the cross-correlation tracking (CCT) and particle tracking algorithms have been tested and proven. Agreement between PIV, LDV, and computational simulations was reasonable.

The three-dimensional PIV algorithms were developed and tested on a flow in a thermal convection enclosure. However, this technique was unsuccessful due to several unresolved difficulties with the 3-D PIV including strong refractive index gradients in the test section.

The nonlinear photorefractive crystal $\mathrm{BaTiO}_{3}$ has been demonstrated as a significant visualizer of turbulent vs. steady-state flow. However, we have identified two potentially significant limitations in that it (1) appears to visualize gradients differently, depending upon the relative orientation of the gradient to the crystal fanning orientation, and (2) visualizes refractive gradients, not absolute phase change, as previously reported in the literature. The desired quantification of photorefractive measurements proved to be very difficult for the reasons discussed above. 


\section{Appendix A- PIV Software Operating Manuals}

\section{A.1.Instructions for Using PIV Software for 2D Analysis}

1) Obtain the images to be analyzed. The images should be in either a tiff (Tagged Image File Format *.TIF) format or a flat format ( 8 bit data, XY format, no header). The images can be captured with a film camera and then scanned into a computer to be stored in the proper format, but usually a CCD camera will be used with a frame grabber board and appropriate software to capture the images directly on a computer. The time between images must be recorded for conversion of measured pixel displacements to actual velocity values. Different time intervals can be used within a sequence, but it is easier to use a constant time interval.

2) Evaluate and adjust the images. This can be done with 4MIP if the images were captured with an EPIX board, or can be done using HALO Desktop Imager 2.2. Using the 4MIP software to evaluate the images has the advantage of saving the images directly to an 8 bit XY flat format which is needed later, avoiding saving the images in the tiff format. In order to use HALO, the images must be read in and saved as tiffs, and then converted to flat format using FLECONV.EXE. Despite this, the HALO software seems to be easier to use than the EPIX software. Either way, the images are to be evaluated and adjusted in order to minimize background noise and maximize the spots. This can be done by adjusting the contrast, brightness, and gamma values in HALO, or by using some of the several image processing techniques in 4MIP. The area to be analyzed must be found and recorded as $x$-start to $x$-final and $y$-start to $y$-final, in pixels. Any areas to be removed should be recorded in the same fashion, as rectangles that block out the unwanted area.

3) Find the spot locations. This is done using FSPOT.EXE, found in the C:IWORK directory. The FSPOT program reads images in flat formats and finds the locations of the spots in the image. The locations are saved to a *.DAT file to be read by the FROM2TO3 program.

4) Convert the spot data from 2D format to 3D format. This is done by FROM2TO3.EXE, in C:IWORK. All this does is read in the XY positions of the spots and save them as XYZ positions with $\mathrm{z}=0$. This allows the $3 \mathrm{D}$ tracking programs to read the data.

5) Track the particles through the images. This can be done with either PST3D.EXE or TP3D.EXE, both found within the C:IWORK directory. Both programs read multiple *.DAT files (PST3D reads 2 frames, TP3D needs at least 4 and can track through several) and output a single *.DI3 file that contains the vectors found.

6) Convert the vector file into a TECPLOT file. This is done using MAKETEC.EXE in C:IWORK. It reads in a *.DI3 file and writes a *.TEC file, a data file ready to be read into the TECPLOT plotting program. The program MAKETEC can be used to weed out some of the 'bad' vectors, using the quality numbers printed out by the tracking programs. 
7) Display the vectors. This can be done using TECPLOT, found in the TECPLOT directory. TECPLOT can be used to view and print the vectors saved using MAKETEC. In addition, a new zone can be created and TECPLOT will interpolate the vectors onto a regular grid. The interpolated data can be smoothed to remove the effect of bad vectors. Using the time between frames and the distance per pixel, the displacement vectors can be changed into velocity vectors. Streamlines can be obtained from the interpolated data.

\section{A.2. Instructions for Using PIV Software for 3D Analysis}

1) Obtain the images to be analyzed. The images should be in either a tiff (Tagged Image File Format *.TIF) format or a flat format ( 8 bit data, $\mathrm{XY}$ format, no header). It is recommended that three cameras be used to capture the data, but 3D analysis can be done with only two. The images can be captured with a film camera and then scanned into a computer to be stored in the proper format, but usually a CCD camera will be used with a frame grabber board and appropriate software to capture the images directly on a computer. The time between images must be recorded for conversion of pixel displacements to actual velocity values. Different time intervals can be used within a sequence, but it is easier to use a constant time interval.

2) Obtain calibration images. In order to combine the 2D images into 3D space, the locations of the cameras must be found. This is done with calibration images. At least six different points must be found with known world and camera points. These points should be within the substance that contains the particles to be tracked in order to compensate for refraction. The cameras cannot be moved until all the data images and the calibration images are captured, since this would give wrong positions for combining the camera images. For the magma chamber experiment, diode lasers were used to find these points. A beam from a laser on the top of the tank with known XZ position was crossed by an orthogonal beam from the side of the tank with known Y position. The place where the beams crossed was used as a calibration point with known world position. An image was taken with the cameras, from which the camera $\mathrm{X}$ and $\mathrm{Y}$ were found. This was done in 8 different positions (the corners of a cube) to get 8 calibration points ( 6 needed, 2 extra for checking). Another method is to place a dark card with light points on it at known XY positions in the flow. The card is then placed at different $Z$ positions to get the necessary calibration points. Note: The world axes must be orthogonal to each other. Also, cameras cannot be placed parallel to the $Y$ axis, so avoid putting cameras straight up or straight down. (Due to a quirk in the math, there is no solution for this angle. Of course, one can simply define a different axis to be the $Y$ axis instead.)

3) Calibrate the cameras. This is done using CAMSOL.EXE, found in C:IWORK and on SAHP053 HP Workstation in the JAHENDER user directory. The CAMSOL program reads in the positions of the calibration points and outputs the positions of the cameras to be read by the CAMCOMB program. This step takes a while (order of days). 
4) Evaluate the images. This can be done with 4MIP if the images were captured with the EPIX boards, or can be done using HALO Desktop Imager 2.2, which is found in Windows. Using the 4MIP software to evaluate the images has the advantage of saving the images directly to an 8 bit $\mathrm{XY}$ flat format which is needed later, avoiding saving the images as tiffs. In order to use HALO, the images must be read in and saved as tiffs, and then converted to flat format using FILECONV.EXE, found in C:UMAGES on the PIV Computer. Despite this, the HALO software seems to be easier to use than the EPIX software. Either way, the images are to be evaluated and adjusted in order to minimize background noise and maximize the spots. This can be done by adjusting the contrast, brightness, and gamma values in HALO, or by using some of the several image processing techniques in 4MIP. The area to be analyzed must be found and recorded as $\mathrm{x}$-start to $\mathrm{x}$-final and $y$-start to $y$-final, in pixels. Any areas to be removed should be recorded in the same fashion, as rectangles that block out the unwanted area.

5) Find the spot locations. This is done using FSPOT.EXE, found in C:IWORK. The FSPOT program reads images in flat formats and finds the locations of the spots in the image. The locations are saved to $\mathrm{a}$ *.DAT file to be read by the FROM2TO3 program.

6) Combine the 2D spot data into 3D data. This is done by CAMCOMB.EXE, in C:IWORK. This reads in the camera positions from CAMSOL and the spot locations from FSPOT to find the position of the spots in 3D.

7) Track the particles through the images. This can be done with either PST3D.EXE or TP3D.EXE, both found within the C:IWORK directory. Both programs read multiple *.DAT files (PST3D reads 2 frames, TP3D needs at least 4 and can track through several) and output a single *.DI3 file that contains the vectors found.

8) Convert the vector file into a TECPLOT file. This is done using MAKETEC.EXE in C:IWORK. It reads in a *.DI3 file and writes a *.TEC file. The program can be used to weed out some of the 'bad' vectors, using the quality numbers printed out by the tracking programs.

9) Display the vectors. This can be done using TECPLOT, found in the TECPLOT directory. TECPLOT can be used to view and print the vectors saved using MAKETEC. In addition, a new zone can be created and TECPLOT will interpolate the vectors onto a regular grid. The interpolated data can be smoothed to remove the effect of bad vectors. Using the time between frames and the distance per pixel, the displacement vectors can be changed into velocity vectors. Streamlines can be obtained from the interpolated data. 


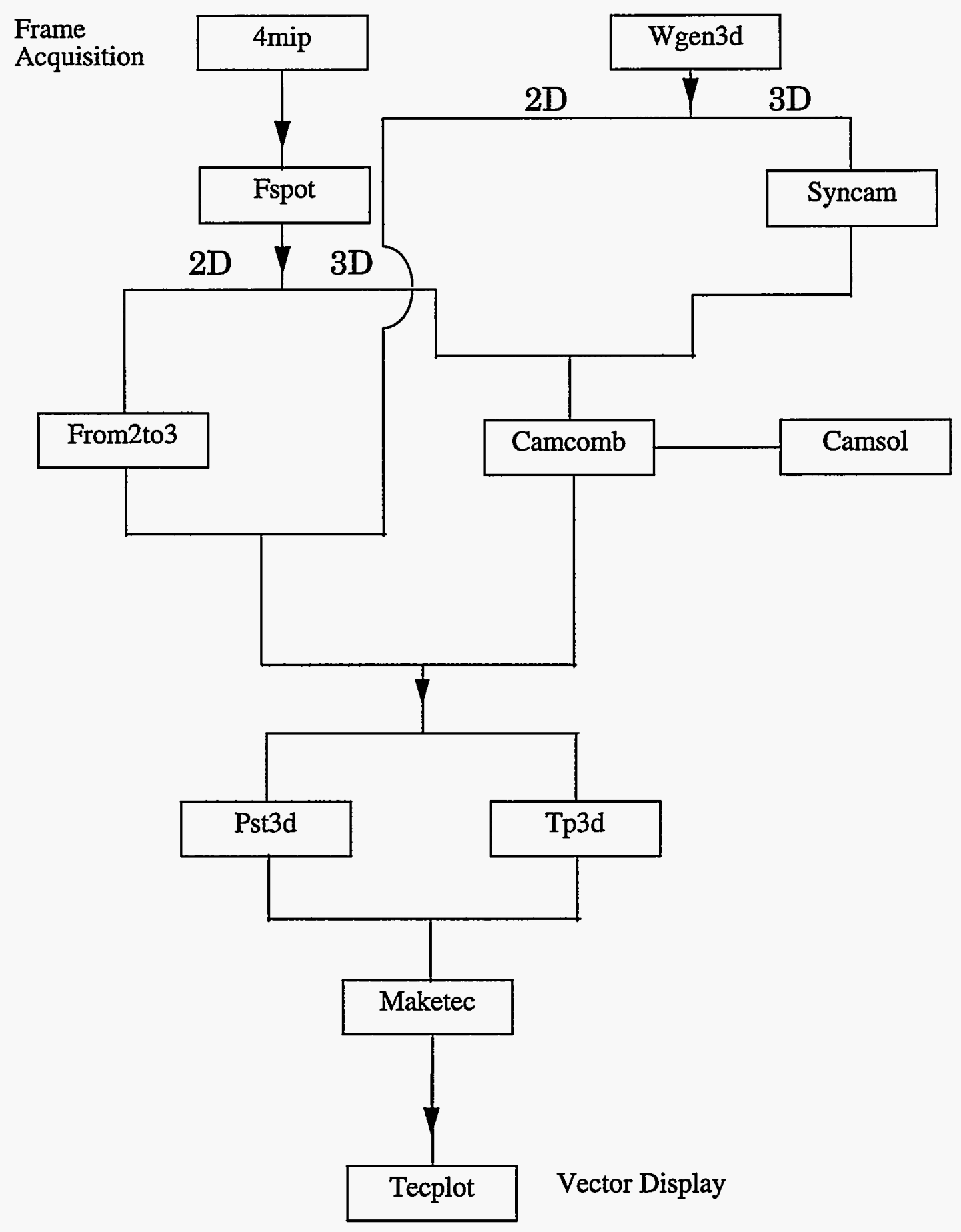

Figure A.1 Basic Outline for PIV software for 2D and 3D analysis 


\section{Appendix 2: 2D and 3D PIV Software}

\section{Detailed Descriptions}

\section{MIP}

4MIP is a commercial image capture/processing software package made to be used with the EPIX frame grabber boards. It has its own manuals, so no details will be given here. In short, 4MIP can be used to capture images from multiple boards, perform image processing on the images, and make measurements of objects in the images.

\section{CAMCOMB(Camera Combination Program)}

CAMCOMB is a program that takes the spots found on images taken simultaneously on two or three cameras and combines them into particle locations in three-dimensional space. In order to do this, it requires the locations of the cameras as outputted by the CAMSOL program. The program requires four or five files to be present in the same directory in order to run correctly. The first is the input deck, named CAMCOMB.INP. The input deck simply contains the names of the other files needed, and is structured as follows:
PARAMFILE
(Camera position and combination parameter file)
INPUTFILE\#1 (*.DAT)
INPUTFILE\#2 (*.DAT)
INPUTFILE\#3 (*.DAT)
(Data points file for first camera)
(Data points file for second camera)
(Data points file for third camera, if 3 cameras)

The parameter file contains the number and positions of the cameras. It also contains information on the area of interest and maximum distance between possible spots. If combined spots are outside the area of interest, or are separated by more than the maximum distance, then they are ignored. This file is created by the CAMSOL program and is named PARAM.DAT unless renamed by the user. The input files contain the spot locations as found from each camera, and must be in the same order as the cameras in the parameter file (otherwise the program will use the wrong camera for the spots). These files are created by the FSPOT program and are named by the user when using FSPOT. Typically they have the *.DAT suffix. The program outputs 2 files; an output file and a data file. Both files contain the location of the particles in three-dimensional space. The output file is named CAMCOMB.OUT and is structured for easier reading by humans. The data file is named CAMCOMB.DAT and is structured to be read as a single frame by the tracking programs, TP3D and PST3D. The data file should be renamed for use appropriately; FRAME001.DAT, for example.

\section{CAMSOL(Camera Calibration and Solution Program)}

CAMSOL is a program that finds the location of two or three cameras based on the positions of calibration spots in each camera and in the world. Each calibration spot must be measured accu- 
rately and must be seen in each camera. The program requires three or four files to be present in the same directory in order to run correctly. The first is the input deck, named CAMSOL.INP. The input deck contains user parameters and the names of the other files needed, and is structured as follows:

NC
SX1,SX2,SX3
CAMSTEP,CAMERR
WORSTEP,WORERR
MINSTEP,MAXIT
XMIN,XMAX
YMIN,YMAX
ZMIN,ZMAX
DMAX
INPUTFLE\#1 (*.DAT)
INPUTFILE\#2 (*.DAT)
INPUTFILE\#3 (*.DAT)

(Number of cameras, 2 or 3 )

(Scaling factors in $\mathrm{X}$ to be used for the cameras, cu/wu)

(Initial step size and max. error for camera coordinates, $\mathrm{cu}$ )

(Initial step size and max. error for world coordinates, wu)

(Minimum step size and maximum number of iterations)

(Area of interest in world coordinates, wu)

(Area of interest in world coordinates, wu)

(Area of interest in world coordinates, wu)

(Maximum distance between possible spots, wu)

(Calibration points file for first camera)

(Calibration points file for second camera)

(Calibration points file for third camera, if 3 cameras)

The various factors are measured in camera units (cu) and world units (wu). These units can be anything consistent, but will usually be pixels and millimeters, respectively. Since there is an infinite number of possible solutions for any set of cameras, the $\mathrm{x}$ scaling factors are entered by the user to select a single solution. The program uses a numerical solver that uses partial differentiation to try to correct for error in the calibration point measurements. The user can control the solverusing the *STEP, *ERR, and MAXIT variables. If any of these variables (except MINSTEP) is set to zero, then numerical correction will not be performed. The *MN and *MAX variables are passed on in the output to be used by the CAMCOMB program. The input files contain the calibration spot locations as found in each camera and the world, and are created by the user. One must be created for each camera, and the spot locations must be in the same order in each input file. The same number of points should be used for each camera. The input files are structured as follows:

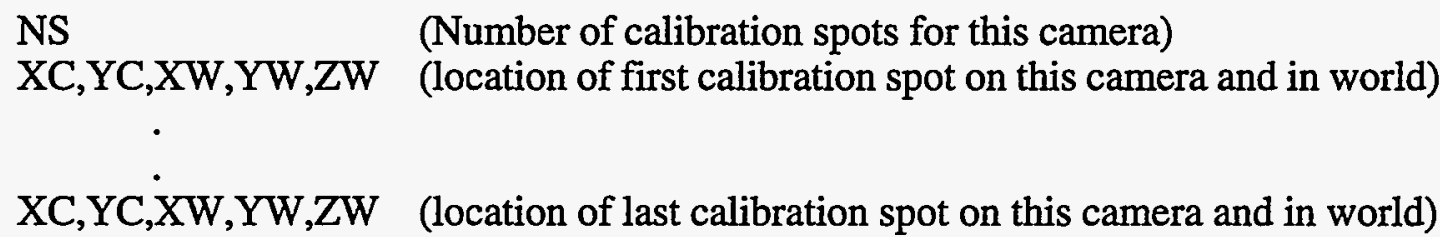

The camera locations $(\mathrm{XC}, \mathrm{YC})$ are found in the image, and are measured in camera units. The world locations (XW,YW,ZW) are measured in the experiment in world units. The program needs at least six calibration points to work, and uses any extra points to help check the locations of the camera after solving for them. The program outputs 5 files on the PC, 6 files on the HP. Two of the files are the output file and the data file. Both of these files contain the positions of the cameras. The data file is named PARAM.DAT and is structured to be read by the CAMCOMB program. The output file is named CAMSOL.OUT and it is structured to be read by humans. It also shows the original and shifted positions of the particles, as well as the error distance between them. Along with these, the HP also creates a file named CAMSOL.SCR, this is simply what 
CAMSOL on the PC normally prints to the screen. The last three files are namedLPARM1.DAT, LPARM2.DAT and LPARM3.DAT. These files contain the shifted position of the calibration particles. They can be used to change the input files to continue iterations where the run that created them stopped. Since these files are recreated after each iteration, even if the program stops because of power failure or program error, the program can be restarted and iterations can be continued. If iterations are continued, be sure to lower the *ERR variables in the input deck, since the spot locations may have already shifted by the initial amount.

\section{FROM2TO3 (From Two-Dimensions to Three-Dimensions Program)}

FROM2TO3 is a simple program that takes two-dimensional spot data produced by the FSPOT program and converts it to three-dimensional spot data capable of being read by either of the tracking programs, PST3D or TP3D. Input is interactive, the program asks what the name of the file to be changed is and what to name the file being created. While the data is being converted, a number of other changes can be made. The program will ask for an angle to rotate the data by, enter 0 if no rotation is desired. It will also ask by how much the image is to be shifted, enter 0,0 if no shift is desired. The program will then ask the start and final coordinates of the image. This can be used to break a seed-dense image into two smaller images by giving halfway marks as the final coordinate on the first run and then as start coordinates on a second run. If the entire set of data is to be transformed, then make sure the start and final coordinates are larger than the original image the data was obtained from. Finally, the program asks for a coordinate multiplier, if no multiplication is desired, enter 1. Note that the transformations are performed in the following order:

1) shift position of data

2) rotate data

3) multiply data

4) drop data if outside of start and final coordinates

5) add $\mathrm{z}$ coordinate, equal to zero

After transforming data, the program asks if you wish transform some more. Enter 1 to transform a new frame of data, or enter 2 to transform the same data again (if breaking the data into two frames, for example). Any other number will quit the program.

\section{FSPOT (Find Spots Program)}

FSPOT is a program that examines an 8-bit flat image and identifies the spot locations on the it. A flat image is simply an image file with no header, with the pixel information in XY order. The 8bits represent 256 gray-scale levels for each pixel. 8-bit flat images can be created by $4 \mathrm{MIP}$, or by converting another image format using the FILECONV program. The FLATTEN batch file, found in the C:IWORK directory, uses the FILECONV program to convert tiff images in a directory into flat images. To use flatten, type it and the complete directory name where the tiff files are located, i.e., 'C:>FLATTEN IWORKICAL0801' makes flat images for every tiff image found in the C:IWORKICAL0801 directory. FSPOT requires the image files and an input deck file to be in its directory to run. Input can be interactive or through the input deck, since FSPOT will edit its input 
deck if ran interactively. There are two possible command line inputs also. If '-b' is entered after the FSPOT command, i.e., 'C>FSPOT - $b$ ', then FSPOT will run in batch mode, using the input deck as is and asking for absolutely no input by keyboard. If - $i$ is entered after the command, and is followed by a colon and a filename, then that file will be used as the input deck. These two commands can be used together, 'C>FSPOT -b -i:TEMP.INP', for example, will use TEMP.INP as the input deck and will run without user input from keyboard. An input deck for FSPOT is structured as follows:

$\begin{array}{lll}\text { INPUTFILE } & \text { (*.FLA) } & \text { (Image file to be analyzed) } \\ \text { OUTPUTFILE (*.OUT) } & \text { (Desired name or "DEFAULT") }(* 1) \\ \text { DATAFILE } & \text { (*.DAT) } & \text { (Desired name or "DEFAULT") }(* 1) \\ \text { TECFILE } & (* . T E C) & \text { (Desired name, "NONE", or "DEFAULT") }(* 1),(* 2) \\ \text { HISTOFILE } & \text { (*.HIS) } & \text { (Desired name, "NONE" or "DEFAULT") }(* 1),(* 2) \\ \text { PICTYPE } & \text { ("epix", can use just "e") } \\ \text { LOCALSIZE } & \text { (Local thresholding region size, cu) }(* 3) \\ \text { LEVEL1,LEVEL2 } & \text { (Zero level and upper level for thresholding, greylevel) (*4) } \\ \text { TOTALX,TOTALY } & \text { (Total horizontal and total vertical size, cu) } \\ \text { STARTX,STARTY } & \text { (Starting location to use in processing, cu) } \\ \text { FINALX,FINALY } & \text { (Final location to use in processing, cu) } \\ \text { WIDTH,HEIGHT } & \text { (Width and height of a frame in world units, for reference) } \\ \text { SMTHLO,SMTHHI } & \text { (Low and high number of pixels for smoothing calculations) } \\ \text { MINAREA,MAXAREA } & \text { (Minimum \& maximum number of pixels to keep spot) } \\ \text { TIME } & \text { (Time in seconds between frames, for reference) } \\ \text { NBURN } & \text { (Number of burnspots to be removed) (*5) } \\ \text { BURNX,BURNY } & \text { (Location of burnspot, cu) } \\ \text { NBUB } & \text { (Number of bubbles for 1/r^2 thresholding) }(* 5),(* 6) \\ \text { BUBX,BUBY } & \text { (Bubble centroid location, cu) } \\ \text { BUBR,BUBTHRSH } & \text { (Bubble radius \& threshold within radius, cu, greylevel) } \\ \text { NAREA } & \text { (Number of rectangular areas to remove) (*5) } \\ \text { AXS,AXF } & \text { (Start x and final x of area to remove, cu) } \\ \text { AYS,AYF } & \text { (Start y and final y of area to remove, cu) } \\ \text { ANSWER } & \text { (Do another file (y/n)?) }\end{array}$

***Notes on Inputdeck***

(*1) if "DEFAULT" is typed as file name (No quotes, but must be in capital letters) file name is <Inputfilename Prefix $><$ Appropriate Suffix $>$. The appropriate suffixes are: *.OUT, .*.DAT, .*.TEC, \& *.HIS for output, data, tecplot, and histogram files, respectively.

(*2) if "NONE" is typed as file name (Same format as in *1, above) then no file of that type is produced for this set of data

(*3) if 0 is entered, constant thresholding is used

(*4) Only used for constant thresholding, but must be entered

(*5) 0 for none

(*6) Not performed if local thresholding is used 
Indented entries are repeated (as a group) a number of times equal to the last non-indented entry above them. If that entry was 0 , then nothing should be entered for the indented entries. If the answer is " $y$ " the same format is duplicated until the answer is " $n$ ". However, it is recommended that FSPOT be used interactively until the user is familiar with the various options, since the interactive selections have more details on the options (besides, it was-a lot of typing). The FSPOT program produces two to four output files. The output file and data file are always created, and both contain information on the spots located. The data file is formatted to be read by the CAMCOMB or FROM2TO3 programs, while the output file is formatted for easy reading. The TECPLOT file, if produced, is structured to be read as a contour plot for TECPLOT. It is simply a binary image showing the spots found. The histogram file, if produced, is simply the number of pixels in the images at each gray level. It can be used with any graphing routine that reads ASCII to show the histogram of the original image

\section{MAKETEC (Make Tecplot File Program)}

MAKETEC is a simple program that takes a vector file produced by TP3D or PST3D (typically with a *.DI3 suffix) and creates a file readable by TECPLOT from it. It can also make a 256 level contour plot for TECPLOT from a two-dimensional flat 8-bit image. Input is interactive, the program asks the user for the name of the input file and the type of file it is (output from TP3D, output from PST3D, or a two-dimensional image). It then creates a TECPLOT ASCI file of the same name, but with the *.TEC suffix. Depending on the type of file being analyzed, furtherinput is required. For a TP3D file, a sigma value cutoff is required. A good track produced by TP3D has a sigma value close to zero, so this cutoff will drop any tracks with sigma values greater than it. For a PST3D file, good vectors have a $\mathrm{Cij}$ value close to one and a large pair confidence $(\mathrm{PC})$ value. Therefore, the program requires $\mathrm{Cij}$ and $\mathrm{PC}$ cutoffs, and will drop any vector with lower values than these. It also requires a velocity cutoff, which generally should have the same value as the search region for PST3D. If a two-dimensional flat image is being converted, then the width and height of the image are required, in pixels. The program also allows some simple manipulation of the image gray level, allowing the user to enter multiplication and addition factors to the gray level. Any gray levels greater than 255 are set to 255 . Finally, the program will ask if another file is to be transformed. Entering 1 will start the program over while any other number will quit.

\section{PST3D (Pick \& Slide Tracking in Three-Dimensions Program)}

The PST3D program compares spot location in two frames of data, and finds the three-dimensional vectors between the two frames. It does this using a method based on the two-dimensional method of cross correlation. PST3D requires an input deck and two spot data files as input. The input deck is named PS3.PIN and should be in the same directory as PST3D when ran. It is structured as follows:

NF

INPUTFILE1 (*.DAT)

INPUTFILE2 (*.DAT)

$\mathrm{XS}, \mathrm{YS}, \mathrm{ZS}$

$\mathrm{XF}, \mathrm{YF}, \mathrm{ZF}$
(Number of frames, must be 2 for this version)

(First spot data file)

(Second spot data file)

(Starting position to be used in processing, wu)

(Final position to be used in processing, wu) 
DX,DY,DZ

DXVS,DYVS,DZVS

IPPN
(Candidate region half-widths, wu)

(Dynamic region starting half-widths, wu)

(Initial possible pair number)

(recall "wu" stands for "world units.") This version of PST3D is not set up for single frame tracking (autocorrelation), e.g., double exposure photography, so the number of frames must always be two. The input files contain the spot locations as produced by either the CAMCOMB program or the WGEN3D program. The starting and final positions, and the half-widths, should be measured in the world coordinates used for the calibration points. The candidate region half-widths are used to find possible pairs between the frames, and should be based on the maximum velocity possible in the volume of interest. If the value is too low, then the correct vectors will not be found; if too large, then the running time is increased, and chances of finding an incorrect vector is increased. The dynamic region starting half-widths are used to determined the comparison volume between the two frames for each spot. This volume will increase in order to encompass at least ten spots in the second frame to compare with. The initial possible pairs number is used for array allocation on the PC, if it's too small the program will increase it and start over, using up more time, but if it's too large the PC may run out of memory, crashing the program. If the program tries to increase the IPPN and crashes, then there may be too many possible vectors to analyze. Either decrease the $\mathrm{dx}$ and dy, or split the image into sections for separate analysis. Output is to written to four files. A data file named PS.DI3 is created and contains the information on the best vectors found. It is formatted to be read by the MAKETEC program. The output file, PAIRCON.DAT, is structured for reading by humans. It contains information on all the possible vectors. Two other files, CIJ.DAT and PC.DAT, contain the distribution of the $\mathrm{Cij}$ and $\mathrm{PC}$ values, respectively. They can be plotted and examined in order to determine the best cutoff values.

\section{SYNCAM (Synthetic Camera Program)}

SYNCAM is a program that takes the synthetic three-dimensional data produced by the WGEN3D program and transforms it into two-dimensional data as viewed by a camera. Input is by input deck, which names the data file to be read. The input deck is named SYNCAM.INP, and is structured as follows:

$\begin{array}{ll}\text { INPUTFILE (*.DAT) } & \text { (Data file containing spot locations) } \\ \text { NC } & \text { (Number of cameras to be modeled, 1-3) } \\ \text { TH,AL,BE } & \text { (Camera rotation angles, for first camera, radians) } \\ \text { DX,DY,DZ } & \text { (Shift from world origin to first cameras origin) } \\ \text { SX,SY,SZ } & \text { (Scaling factors for first camera) }\end{array}$

(Repeat last 3 lines for 2nd and 3rd camera, if necessary)

For each camera modeled, two files are produced. The first is a file named SYNCAM\#.DAT (where \# is the number of the camera) which contains the two-dimensional data in the same format as a data file created by the FSPOT program. The second is named CALIB\#.DAT, and can be used as an input file for the CAMSOL program. In addition, a file named SYNCAM.OUT is produced, 
which simply contains the positions of all the cameras being modeled for later comparison with the CAMSOL program.

\section{TECPLOT}

TECPLOT is a commercial software package made for all sorts of data analysis. Since it has its own manuals, no details will be given here. TECPLOT can be used for making graphs, drawing vectors (2D or $3 \mathrm{D})$ and representing images as contour plots.

\section{TP3D (Track Particle in Three-Dimensions Program}

TP3D compares spot locations in four or more frames of data, and finds tracks throughout all the frames. It does this by comparing the possible tracks to paths of constant curvature. It requires an input deck file to tell it the names of the four to fourteen input data files, along with search radius information. The input deck is named TP3D.TIN, and is simply structured as follows:

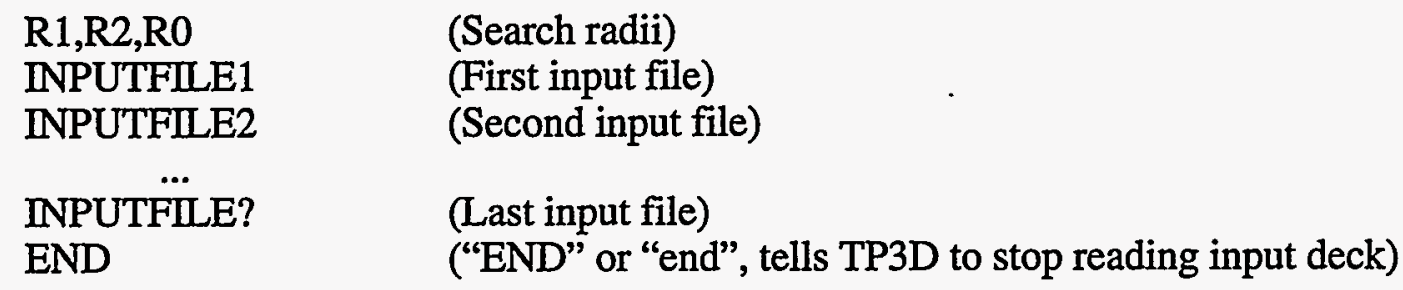

The search radii are used to determine which particles to create tracks with. Similar to the halfwidths for PST3D, it's important to get their values in the right range. R1 is the search radius for the second particle in a track, and its value should be the maximum velocity in the frames. $R 2$ is the search radius for the particles after the second frame, and should be the maximum acceleration in the frames. $\mathrm{R} 0$ is the minimum search radius for the second particle, and should be the minimum velocity in the frames. The input files contain the locations of the spots in three-dimensional space, as created by either the CAMCOMB program or the WGEN3D program. Output is to three files. The first is TP3D.DI3, which contains the best track information, and is structured to be read by the MAKETEC program. The other two, named TP3D.FNL and TP3D.XYS, contain the track information in different formats.

\section{WGEN3D (Working Generate Three-Dimensional Data Program)}

WGEN3D creates a series of frames of synthetic three-dimensional data, which can be tracked to test the other 3DPIV programs. Input is by hand, and no data files are needed for the program. The program can create from 2 to 14 frames of data. From 1 to 10000 spots can be randomly distributed across the first frame of data. These spots are then moved across the other frames by numerical equations representing flow around a sphere, spray flow, or reverse drain flow. A freestream ve- 
locity is required. The initial seed placement can be restricted in order to prevent seeds from leaving the area of interest and being lost from later frames. Finally program asks the user to define the area of interest, by asking for a $\mathrm{r}$ (ange) and a c(onstant). With this information, the program defines a cube with each side being $r$ units long, shifted along all axes $c$ amount. There is a data file created for each frame, containing the spot locations in a format similar that produced by the CAMCOMB program. These files are named GEN3DF\#.DAT, with \# being the number of the frame. In addition, two other files are created describing the flow. The first is GENSTATS.DAT, which just describes the user's selections for creating the data. The second is GEN3D.DI3, which is formatted like output from the TP3D program, and can be used to compare with the results of the tracking programs. It can also be used with MAKETEC to create a vector plot in TECPLOT. 


\section{References}

Adrian, R. J., 1991, "Particle Imaging Techniques for Experimental Fluid Mechanics," Annual Review of Fluid Mechanics, 23, Annual Reviews, Inc., Palo Alto, CA.

Albrecht, G. F., Robey, H. F., and Moore, T. R., 1990, "Optical Technique for the Measurement of Turbulent Spectra Using the Photorefractive Properties of $\mathrm{BaTiO}_{3}$," Applied Physics Letters, 57 (9), 864-866.

Blanchat, T. K., 1991, "Development of Pulsed Laser Velocimetry Techniques for Measurement of Two-Phase Interfacial Drag in a Horizontal Stratified Flow," Ph.D. Thesis, Texas A\&M University, College Station, TX.

Buchhave, P., and Jakobsen, M. L., 1990, “Optical Correlator for PIV Image Processing," Proceedings of the 3rd International Conference on Laser Anemometry, 609-616.

Chu, T. Y., and Hickox, C. E., 1990, "Thermal Convection with Large Viscosity Variation in an Enclosure with Localized Heating," J. Heat Transfer 112, 388-395.

Chu, T. Y., Dunn, J. C., Finger, J. T., Rundle, J. B., and Westrich, H. R., 1990, “The Magma Energy Program," Geothermal Resources Council Bulletin, 19 (2), 42-52.

Durst, F., Melling, A. and Whitelaw, J. H., 1981, Principles and Practice of Laser-Doppler Anemometry, Second Edition, Academic Press, New York, p. 369.

Fluid Dynamics International, 1993, FIDAP Users Manual Revision 7.0, Fluid Dynamics International, Evanston IL.

Gonzales, R. C., and Wintz, P., 1987, Digital Image Processing, Ch. 2.5, 36-54.

Grant, I., ed., 1994, Selected Papers on Particle Image Velocimetry, SPIE Volume MS99, The Society of Photo-Optical Instrumentation Engineers.

Hassan, Y., and Blanchat, T., 1991a, "Full-Field Bubbly Flow Velocity Measurements by Digital Image Pulsed Laser Velocimetry," Experiments in Fluids, 11, 293-301.

Hassan, Y., and Blanchat, T., 1991b, "Flow Velocity Measurements Using Digital Image Pulsed Laser Velocimetry," Optical Engineering, 30 (8), 1220-1227.

Henderson, J. A., Masters thesis, Texas A\&M University, 1994.

Hickox, C. E., and Chu, T. Y., 1991, "Developing Natural Convection in a Fluid Layer with Localized Heating and Large Viscosity Variation," in FED Vol. 107, General Topics in Fluids Engineering, edited by F. M. White and K. Kamemoto (American Society of Mechanical Engineers, New York), 75-81. 
Iwatsu, R., Ishii, K., Kawamura, T., Kawamura, K., and Hyun, J. M., 1989, "Numerical Simulation of three-dimensional flow structure in a driven cavity," Fluid Dynamics Research, 5, 173189.

Koseff, J. R., and Street, R. L., 1984a, "Visualization Studies of a Shear Driven Three-Dimensional Recirculating Flow," J. Fluids Engineering, 106, 21-29.

Koseff, J. R., and Street, R. L., 1984b, “On End Wall Effects in a Lid-Driven Cavity Flow," J. Fluids Engineering, 106, 385-389.

Koseff, J. R., and Street, R. L., 1984c, "The Lid-Driven Cavity: A Synthesis of Qualitative and Quantitative Observations," J. Fluids Engineering, 106, 390-396.

Lourenco, L. M., Krothapelli, A., and Smith, C. A., 1989, "Particle Image Velocimetry," in Advances in Fluid Mechanics Measurements, Gad-el-Hak, M., editor, Springer-Verlag, Berlin, 128197.

Marrakchi, A., Petersen, P. M., and Buchhave, P., 1991, "Photorefractive Particle Image Velocimetry," Optics and Photonics News, December, 16-17.

Merzkirch, W., 1987, Flow Visualization, Academic Press, Inc., Orlando, FL.

O’Hern, T. J., Torczynski, J. R., Blanchat, T. K., Chu, T. Y., and Tassin, A. L., 1994, "Shear-Driven Flow in a Square Cavity: A Comparative Study Using LDV, PIV, and Computational Simulations," ASME Symposium on Laser Anemometry: Advances and Applications, T. T. Huang and M. V. Otugen, eds. (SAND93-1707C)

O'Hern, T. J., and Tassin, A. L., 1993, "Examination of a Shear-Driven Cavity Flow Using LDV and PIV," Bull. Amer. Phys. Soc., 37 (10).

O'Hern, T. J., Robey, H. F., Torczynski, J. R., Neal, D. R., and Shagam, R. N., 1991, “Measurements of Passive-Scalar Spectra for Grid Turbulence Using Nonlinear Optical Technique," Proceedings of the ASME Fluid Measurements and Instrumentation Forum, 29-33.

O'Hern, T. J., d'Agostino, L., and Acosta, A. J., 1988, "Comparison of Holographic and Coulter Counter Measurements of Cavitation Nuclei in the Ocean," J. Fluids Engineering 110 (2), 200207.

Papamoschou, D., Lin, M., Bunyajitradulya, A, and Robey, H. F., 1993, "Non-Intrusive Technique for Measurement of Power Spectra in Compressible Turbulence" AIAA-93-0633, American Institute of Aeronautics and Astronautics 31st Aerospace Sciences Meeting, January 1993, Reno, NV.

Pepper, D. M., Feinberg, J., and Kukhtarev, N. V., 1990, "The Photorefractive Effect," Scientific American, October, 62-74. 
Reiman, T. C., and Sabersky, R. H., 1968, "Laminar Flow Over Rectangular Cavities," Int. J. Heat Mass Transfer, 11, 1083-1085.

Robey, H. F., 1990, "Spectrum of a Passive Scalar in the Inertial-Convective Subrange of an Anisotropic Flow," Physical Review Letters, 65, 11, 1360-1363.

Robey, H., Albrecht, G., and Moore, T., 1990, "An Optical Technique for the Direct Measurement of the 2-D Spectral Density of a Passive Scalar in Turbulent Flow," AIAA Paper 90-1667 (AIAA, Washington DC).

Siahmakoun, A., and Harrer, T., 1993, "Dynamic Photorefractive Schlieren System," Applied Optics, 32, 7281.

Torczynski, J. R., Henderson, J. A., O’Hern, T. J., Chu, T. Y., and Blanchat, T. K., 1995, “ThreeDimensional Natural Convection of a Fluid with Temperature-Dependent Viscosity in an Enclosure with Localized Heating," National Heat Transfer Conference, HTD-Vol. 305, 81-87.

Torczynski, J. R., and O'Hern, T. J., 1994, “Numerical Simulations of Flow in a Three-Dimensional Cavity-Channel Geometry," ASME Fluids Engineering Division Summer Meeting, General Fluids Engineering, F. M. White, ed. (SAND93-1439C).

Torczynski, J. R., and O'Hern, T. J., 1993, "Numerical Simulations of Three-Dimensional Cavity Flows," Bull. Amer. Phys. Soc., 37 (10). 


\section{Distribution}

MS0826 Dept. 9111 T. J. O’Hern (2)

MS0826 Dept. 9111 S. N. Kempka, actg.

MS0834 Dept. 9112 J. R. Torczynski (2)

MS0834 Dept. 9112 A. C. Ratzel

MS0825 Dept. 9115 V. A. Amatucci

MS0328 Dept. 2674 R. N. Shagam

MS0328 Dept. 2674 J. A. Wilder

MS1139 Dept. 6423 T. K. Blanchat

MS1139 Dept. 6423 T. Y. Chu

MS1139 Dept. 6423 K. O. Reil

MS0188 Dept. 4523 LDRD Office

U. Michigan A. L. Tassin-Leger

MS 9018 Central Technical Files, 8940-2

MS0899 Technical Library, 4414 (5)

MS0619 Review and Approval Desk, 12690 for DOE/OSTI 